\title{
Levelling out differences in aerobic glycolysis neutralizes the competitive advantage of oncogenic PIK3CA mutant progenitors in the esophagus
}

\author{
Albert Herms $^{1 *}$, Bartomeu Colom ${ }^{1 *}$, Gabriel Piedrafita ${ }^{1,2}$, Kasumi Murai ${ }^{1}$, Swee Hoe Ong ${ }^{1}$, \\ David Fernandez-Antoran ${ }^{1}$, Christopher Bryant ${ }^{1}$, Christian Frezza ${ }^{3}$, Bart Vanhaesebroeck ${ }^{4}$, \\ Philip H. Jones ${ }^{1,3 * *}$ \\ ${ }^{1}$ Wellcome Sanger Institute, Hinxton CB10 1SA, UK \\ 2 Spanish National Cancer Research Centre (CNIO), Madrid 28029, Spain \\ ${ }^{3}$ MRC Cancer Unit, University of Cambridge, Hutchison-MRC Research Centre, Cambridge \\ Biomedical Campus, Cambridge CB2 OXZ, UK \\ ${ }^{4}$ UCL Cancer Institute, University College London, London WC1E 6BT, UK \\ * These authors contributed equally to this work \\ ** Corresponding author: pj3@sanger.ac.uk
}




\section{Summary}

Normal human tissues progressively accumulate cells carrying mutations. Activating mutations in PIK3CA generate large clones in the aging human esophagus, but the underlying cellular mechanisms are unclear. Here, we tracked mutant PIK3CA esophageal progenitor cells in transgenic mice by lineage tracing. Expression of an activating heterozygous Pik3ca ${ }^{H 1047 R}$ mutation in single progenitor cells tilts cell fate towards proliferation, generating mutant clones that outcompete their wild type neighbors. The mutation leads to increased aerobic glycolysis through the activation of Hif1 $\alpha$ transcriptional targets compared with wild type cells. We found that interventions that level out the difference in activation of the $\mathrm{PI3K} / \mathrm{HIF} 1 \alpha / a e r o b i c$ glycolysis axis between wild type and mutant cells attenuate the competitive advantage of Pik3ca ${ }^{H 1047 R}$ mutant cells in vitro and in vivo. Our results suggest that clinically feasible interventions that even out signaling imbalances between wild type and mutant cells may limit the expansion of oncogenic mutants in normal epithelia. 


\section{Introduction}

As humans age, normal tissues accumulate clones carrying somatic mutations (Blokzijl et al., 2016; Lee-Six et al., 2018; Martincorena et al., 2018 ; Martincorena et al., 2015; Moore et al., 2020; Yizhak et al., 2019; Yokoyama et al., 2019; Yoshida et al., 2020). An example is the esophagus, in which the normal epithelium develops into a patchwork of mutant clones by middle age (Martincorena et al., 2018). There is genetic evidence that the most prevalent mutations are in genes under strong positive selection, arguing that they confer a proliferative or survival advantage over wild type cells (Hall et al., 2019). However, little is known about the cellular mechanisms that underpin the clonal expansions triggered by most of the mutant genes. Understanding these processes is important as it both reveals genes that regulate progenitor cell behavior and, as most of the selected mutant genes are frequently mutated in cancer, gives insight into the earliest stages of neoplastic transformation. The identification of pharmacologically-druggable mutations may also open opportunities for cancer prevention.

One gene that is recurrently mutated in normal human esophagus is PIK3CA, which encodes the p110 $\alpha$ catalytic subunit of phosphoinositide 3-kinase (PI3K) (Martincorena et al., 2018; Yizhak et al., 2019; Yokoyama et al., 2019). PI3K is a signaling hub activated by insulin and growth factors to regulate a broad range of processes including cell proliferation, survival, growth and metabolism, mainly through the activation of the Akt/mTOR signaling axis (Fruman et al., 2017; Madsen et al., 2018). Activating PIK3CA mutations are recurrently found in solid tumors including esophageal squamous cell carcinoma (ESCC), and in overgrowth syndromes and vascular malformations (Castel et al., 2016; Castillo et al., 2016; Consortium, 2017; Madsen et al., 2018).

Reviewing published data, 74 missense PIK3CA mutant clones were identified in $18 \mathrm{~cm}^{2}$ of histologically normal human esophageal epithelium (Martincorena et al., 2018). PIK3CA missense mutations generate particularly large clones in the human esophagus (Figure 1A). Indeed, PIK3CA is the mutant gene with the second highest average Variant Allele Fraction (VAF) (Figure 1B). The VAF corresponds to the proportion of sequence reads detected for each DNA variant and, in diploid cells, is proportional to the size of the mutant clones carrying the mutation in the sample analyzed. These mutant clones were significantly enriched for pathogenic or likely pathogenic PIK3CA activating mutations according to the ClinVar mutation database (https://clinvarminer.genetics.utah.edu/variants-by-gene/PIK3CA); with 47\% (35/74) activating PIK3CA mutations observed, well above the $1 \%$ expected under neutrality $\left(p=2 e^{-}\right.$ 27, two tailed binomial test, see methods) (Martincorena et al., 2018). Notably, mutations classified as pathogenic or likely pathogenic had significantly higher clone size or VAF than 
bioRxiv preprint doi: https://doi.org/10.1101/2021.05.28.446104; this version posted May 28,2021 . The copyright holder for this preprint (which was not certified by peer review) is the author/funder, who has granted bioRxiv a license to display the preprint in perpetuity. It is made available under aCC-BY 4.0 International license.

the remaining PIK3CA missense mutants or the total 603 silent mutations in all tested genes, which have no effect on cell behavior (Figure 1C). The most prevalent pathogenic mutation was PIK3CA ${ }^{H 1047 R}$ (35\% (8/23) of all pathogenic mutation events) (Figure 1D), which had a significantly higher VAF than the other PIK3CA missense mutations (Figure 1E). PIK3CA ${ }^{H 1047 R}$ mutation occurs recurrently in human esophageal and other cancers (https://cancer.sanger.ac.uk/cosmic/gene/analysis?In=PIK3CA), benign overgrowth syndromes and in vascular malformations (Cancer Genome Atlas Research et al., 2017; Castillo et al., 2016; Fruman et al., 2017; Keppler-Noreuil et al., 2014; Madsen et al., 2018). Taken together, these findings indicate that activating PIK3CA mutants, and particularly $P I K 3 C A^{H 1047 R}$, may drive large clonal expansions in normal human esophagus.

Figure 1

A

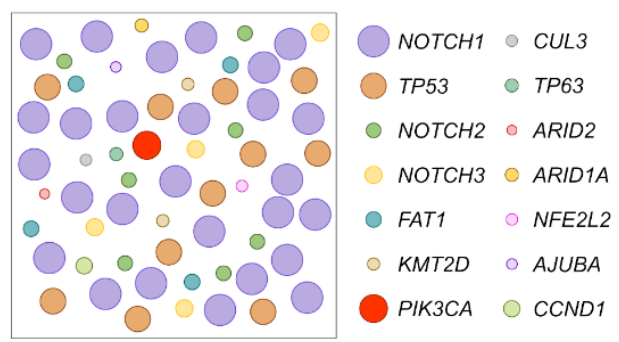

B

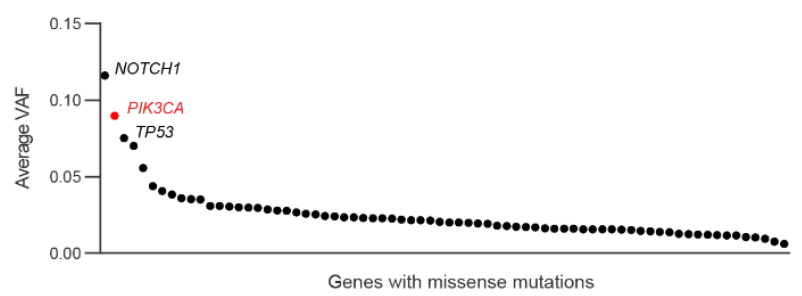

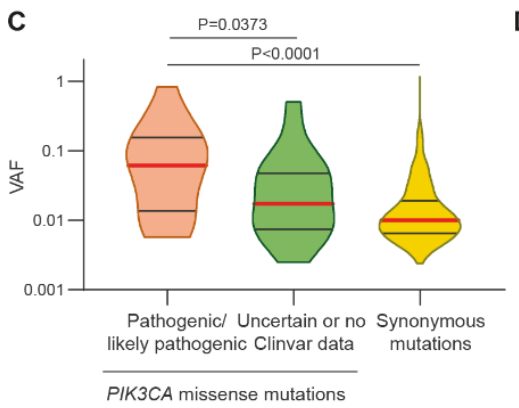

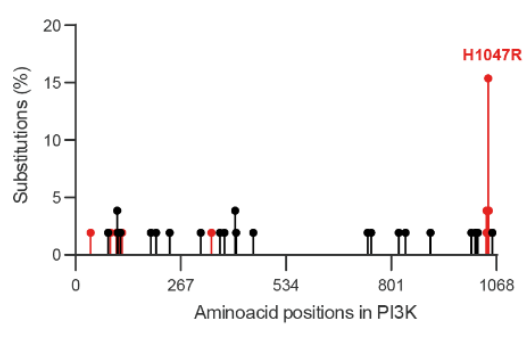

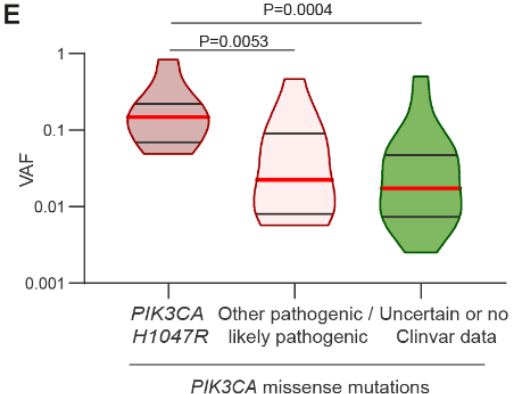

Figure 1. PIK3CA ${ }^{H 1047 R}$ clones have high variant allele frequency in the human esophageal epithelium. Mutant clones in normal human esophagus (data from (Martincorena et al., 2018)).(A) Schematic representation of clones carrying missense mutations under positive selection. Clones are represented as circles, with their frequency and size relative to the mutation frequency and their variant allele frequency (VAF), respectively. VAF is proportional to clone size in diploid normal tissue. (B) Missense mutations detected more than once ordered by decreasing average VAF. VAF of missense mutations in the PIK3CA gene are highlighted in red. (C) Distribution of PIK3CA missense mutations classified according to the Clinvar database (https://www.ncbi.nlm.nih.gov/clinvar/) into pathogenic/likely pathogenic or uncertain significance/no data available. VAF distribution of the synonymous passenger mutations in all genes is also shown. Medians (red) and quartiles (grey lines) are represented. Two-tailed Mann Whitney test. (D) Distribution of codons altered by missense mutations in $p 110 \alpha$ protein. Pathogenic/likely pathogenic mutations are shown in red. (E) VAF distribution of PIK3CA ${ }^{H 1047 R}$ mutations, and other PIK3CA missense mutations classified either as pathogenic/likely pathogenic or uncertain significance/no data available. Medians (red) and quartiles (grey lines) are represented. Two-tailed Mann-Whitney test. 
In light of these observations, we set out to investigate the mechanisms by which Pik3ca ${ }^{H 1047 R}$ mutant progenitors colonize normal esophagus in mouse esophageal epithelium. The tissue consists of layers of keratinocytes, with progenitor cells residing in the deepest, basal cell layer. Differentiating cells exit the cell cycle and leave the basal layer, migrating towards the epithelial surface where they are shed (Doupe et al., 2012; Piedrafita et al., 2020) (Figure 2A). Each progenitor division generates either two progenitor daughters, two non-dividing differentiating cells or one cell of each type (Figure 2B) (Doupe et al., 2012; Piedrafita et al., 2020). The probabilities of these outcomes are balanced across the progenitor population so that one progenitor and one differentiating daughter are produced from an average division. This ensures that across the progenitor population, equal numbers of progenitor and differentiating cells are generated, maintaining tissue homeostasis. Mutations may tilt the balance of cell production towards proliferation driving the clonal expansion of mutant progenitors (Alcolea et al., 2014; Fernandez-Antoran et al., 2019; Murai et al., 2018) (Figure 2B).

To explore the effect of activating Pik3ca mutations on progenitor cells we developed a new transgenic mouse model that allowed us to track the fate of individual progenitors induced to express a single allele of the Pik3ca ${ }^{H 1047 R}$ mutant in a background of wild type cells. We found that this mutation tilts progenitor cell fate, so that excess of mutant progenitors are produced per average division, a proliferative advantage that promotes mutant clone expansion. We show that Pik3ca ${ }^{H 1047 R}$ activates glycolysis via the HIF1a transcription factor in esophageal cells and that levelling the competitive 'playing field' with agents that regulate glycolysis blocks the competitive advantage of Pik3ca ${ }^{H 1047 R}$ mutant cells in vitro and in vivo. 
Figure 2

A

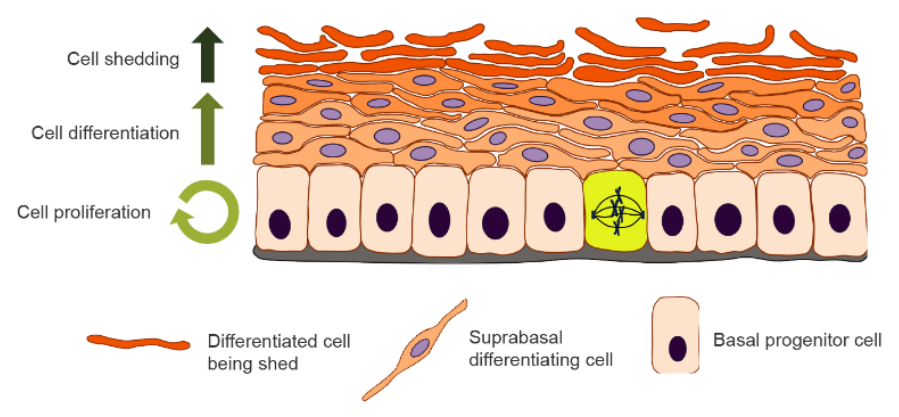

C

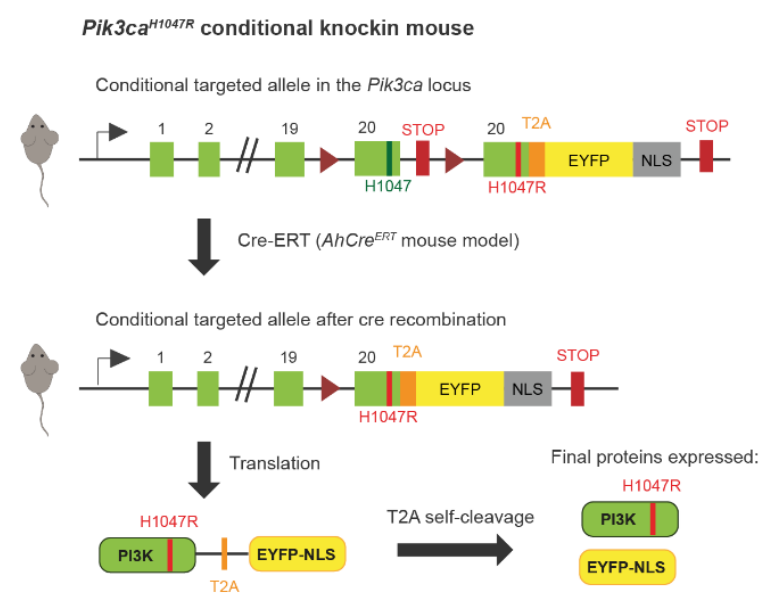

B

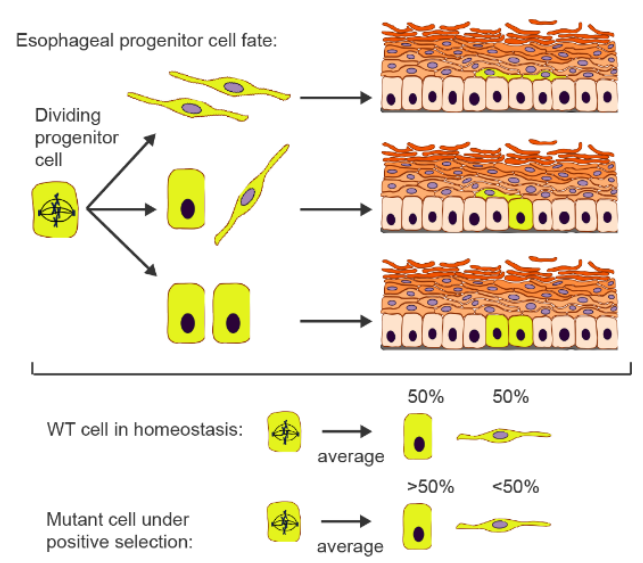

D

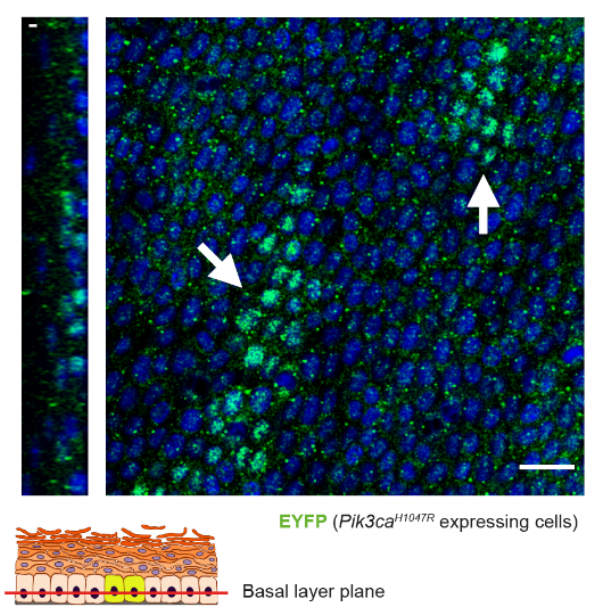

Figure 2. Mouse esophageal epithelium cell behavior and conditional Pik3ca ${ }^{H 1047 R}$ transgenic mouse model. (A) Mouse esophageal epithelium. The lowest basal layer contains proliferating progenitor cells and post-mitotic differentiating cells which will enter the suprabasal cell layers. Differentiating cells migrate through the layers to the tissue surface where they are shed. (B) Each progenitor cell division may generate two differentiating cells, two progenitor cells or one of each type. In homeostasis, the probabilities of each outcome are balanced in order to produce equal numbers of progenitor cells and differentiating cells across the epithelium. Mutations under positive selection may tilt the cell fate towards proliferation, producing excess progenitor cells. (C) Schematic illustration of the conditional targeted allele in the Pik3ca locus. Pik3ca exon 20 was flanked by loxP sites (triangles). The engineered duplicate region of Pik3ca exon 20 contains the H1047R mutation, and sequences coding for a self-cleaving T2A peptide and an enhanced Yellow Fluorescent Protein (EYFP) followed by a nuclear localization signal (NLS). Prior to Cre-mediated recombination, the wild type p110a protein is expressed; however, after Cre mediated recombination, the allele co-expresses $\mathrm{p} 110 a^{\mathrm{H} 1047 \mathrm{R}}$ mutant protein and EYFP-NLS. Cre recombination was mediated by crossing the conditional mutant strain with $A h C r e^{E R T}$ mice which express the Cre recombinase upon treatment with $\beta$-naphthoflavone and tamoxifen. (D) Typical confocal $z$ stack image of a region of an esophageal epithelial whole-mount, viewed top down, from an Ahcre ${ }^{E R T}$ Pik3ca ${ }^{H 1047 R / w t}$ mice 3 months after induction with $\beta$-naphthoflavone and tamoxifen. An optical section through the basal cell layer is shown, Pik3ca H1047R/wt clones are indicated by white arrows. YFP immunofluorescence (green), nuclei are stained with DAPI (blue). Scale bar, $20 \mu \mathrm{m}$. 


\section{Results}

\section{Generation of inducible Pik3ca ${ }^{\text {H1047R-YFP }}$ knock-in mice}

The majority of Pik3ca mutant clones found in the normal esophagus carry an activating H1047R mutation in one allele of the Pik3ca locus. To determine whether induction of a heterozygous Pik3ca ${ }^{H 1047 R}$ allele altered progenitor cell behavior, we developed a new conditional mouse strain, Pik3ca ${ }^{\text {fl-H1047R-T2A-YFP-NLS }}$ (henceforth referred to as Pik3ca ${ }^{\text {H1047R-YFP). }}$ A conditional allele of $P i k 3 c a^{H 1047 R}$, with a nuclear localized Yellow Fluorescent Protein (YFP) reporter linked to the C-terminus of the Pik3ca ${ }^{H 1047 R}$ protein by a T2A self-cleaving peptide (Trichas et al., 2008), was targeted to the Pik3ca locus. Following recombination mediated by Cre recombinase, the wild type exon 20 of Pik3ca is excised and replaced by the mutant exon 20 encoding Pik3ca ${ }^{H 1047 R}$ (Figure 2C). This design allowed us to track individual Pik3ca ${ }^{\text {H1047R- }}$ YFP cells in a Pik3ca ${ }^{\text {wt/wt }}$ background, as the recombined mutant cells and their progeny stain positive for YFP (Figure 2D). Following T2A cleavage, a 20-amino-acid peptide remained at the $C$ terminus of Pik3ca ${ }^{H 1047 R}$ protein. We found that a C-terminally extended $p 110 a^{H 1047 R}$ protein could still activate the PI3K/Akt pathway upon transient transfection in NIH3T3 fibroblasts (Figure S1A) and also induce modest Akt activation upon in vitro recombination of primary esophageal keratinocytes from Pik3ca ${ }^{\text {H1047R-YFP }}$ mice (Figure S1B-F).

Figure S1

A

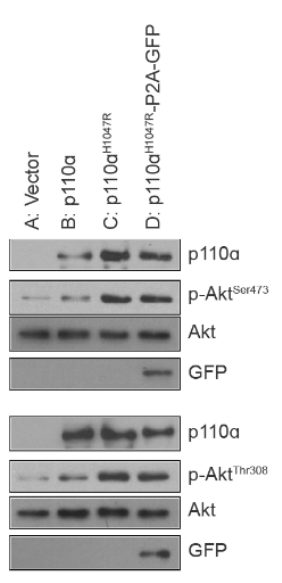

E

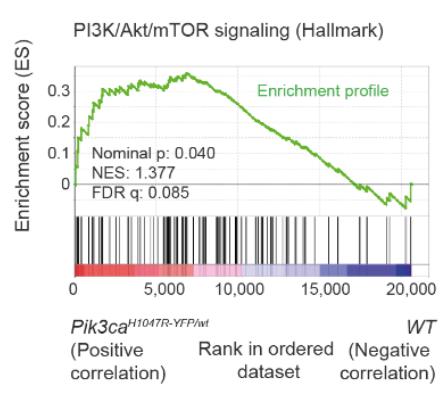

B

C
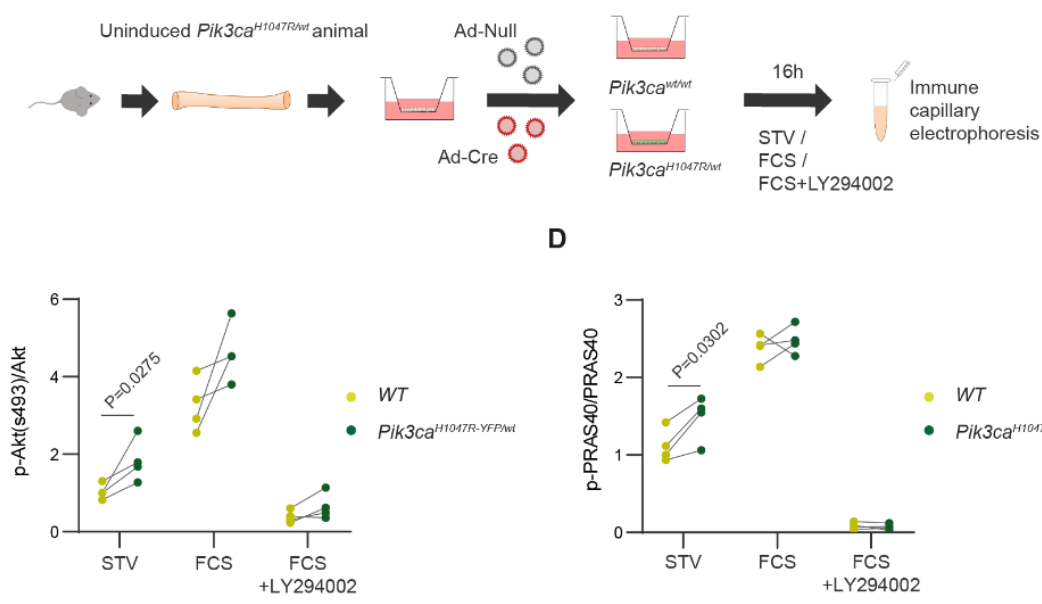

F
D

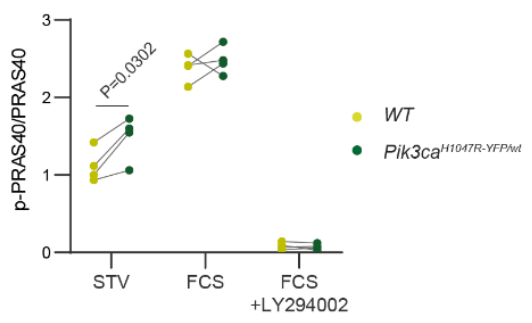

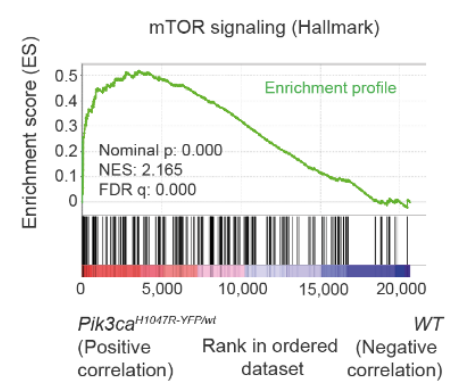


Supplementary Figure 1. Related to Figure 2. Expression of $p 110 \alpha^{H 1047 R}$ mutant protein activates PI3K pathway. (A) Immunoblots of NIH3T3 cells transfected with an empty vector, a vector carrying wild type p110a, p110a $a^{\mathrm{H} 1047 \mathrm{R}}$ or $\mathrm{p} 110 \mathrm{a}^{\mathrm{H} 1047 \mathrm{R}}-\mathrm{P} 2 \mathrm{~A}-\mathrm{GFP}$. Cells were starved for $24 \mathrm{~h}$ and lysed. Expression of PI3K, GFP, AKT and phosphorylation of AKT on Ser ${ }^{473}$ and $\mathrm{Thr}^{308}$ is shown. Images are representative of 3 replicate experiments. (B) Experimental protocol. Primary esophageal keratinocytes were isolated from uninduced Pik3ca ${ }^{H 1047 R-Y F P / w t}$ mice and infected either with null-adenovirus (uninduced Pik3ca ${ }^{\text {wt }}$ wt controls) or Cre-adenovirus (induced Pik3ca ${ }^{H 1047 R-Y F P / w t}$ ). Cells were treated in medium without added growth factors and $0.1 \%$ serum (Starved, STV) or medium with $20 \%$ serum and growth factors (FCS) or FCS plus the PI3K inhibitor LY294002 $50 \mu \mathrm{M}$. Cells were lysed and protein lysates were analyzed by immune capillary electrophoresis. (C-D) Results from immune capillary electrophoresis of lysates from protocol shown in (B). Total AKT protein and phospho-AKT(Ser473) (C) and total PRAS40 protein and phospho-PRAS40 (D). Two-tailed ratio paired $t$-test. $n=4$ biological replicates. (E-F) Gene set enrichment analysis (GSEA) histograms of PI3K/Akt/mTOR and mTOR signaling Hallmark gene sets comparing RNA-seq data from induced Pik3ca ${ }^{H 1047 R-Y F P / w t}$ and uninduced cells from the same animals maintained in minimal FAD medium. The nominal $p$-value, the normalized enrichment score (NES) and the false discovery rate (FDR) q-value are indicated. $n=4$ independent replicates per condition from one animal each.

\section{Pik3ca ${ }^{H 1047 R-Y F P / w t}$ mutant cells outcompete wild type cells in esophageal epithelium}

To track the fate of Pik3ca ${ }^{H 1047 R-Y F P / w t}$ progenitors in vivo, we crossed Pik3ca ${ }^{\text {H1047R-YFP/wt }}$ mice with the inducible Cre recombinase line $A h C r e^{E R T}$. Cre was induced in adult AhCre ${ }^{E R T}$ Pik3ca ${ }^{H 1047 R-Y F P / w t}$ mice (henceforth termed Cre-Pik3ca ${ }^{H 1047 R-Y F P / w t}$ ) at a level that recombined individual esophageal cells, which went on to generate clones of Pik3ca mutant cells (Clayton et al., 2007; Doupe et al., 2012; Kemp et al., 2004). As controls, we induced AhCre ${ }^{E R T}$ Rosa26 fYFP/wt (henceforth termed Cre-RYFP) animals that are Pik3ca wild type and express a neutral YFP reporter after recombination. At multiple time points up to 6 months after induction, animals were culled (Figure 3A), the esophagus collected and the entire epithelium isolated, stained and imaged in 3D using confocal microscopy. The number of cells and their location within individual clones was then counted and control and mutant clone size distributions compared (Table S1).

Within 10 days of induction we observed that the total number of cells in Pik3ca H1047R-YFP/wt clones was larger than that of wild type controls, the difference increasing progressively over 6 months (Figures 3B and C). The mutant clones had a higher proportion of basal cells and fewer differentiated (suprabasal) cells compared with controls (Figure 3D). These observations indicate Pik3ca ${ }^{\text {H1047R-YFP/Wt }}$ cells have a competitive advantage over their wild type neighbors and suggest that mutant progenitors may generate a lower proportion of differentiating and more proliferating progeny than their wild type equivalents. 
Figure 3

A

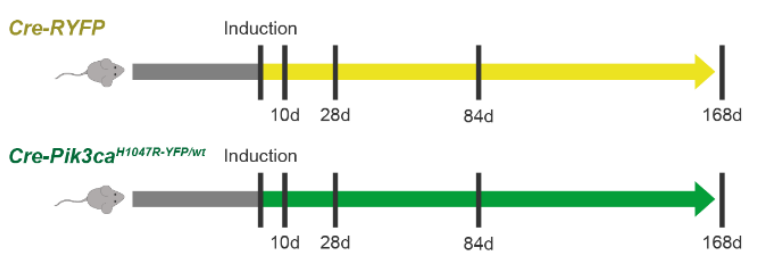

C
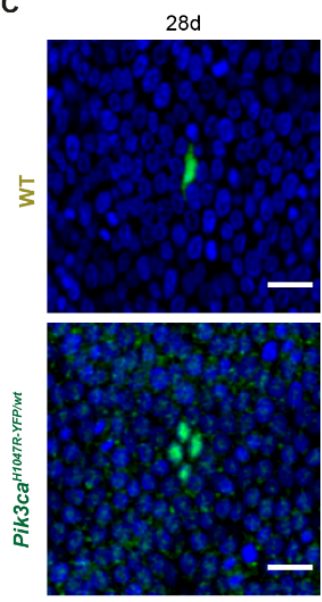

E

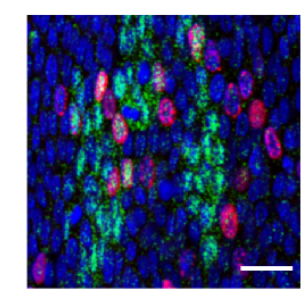

F
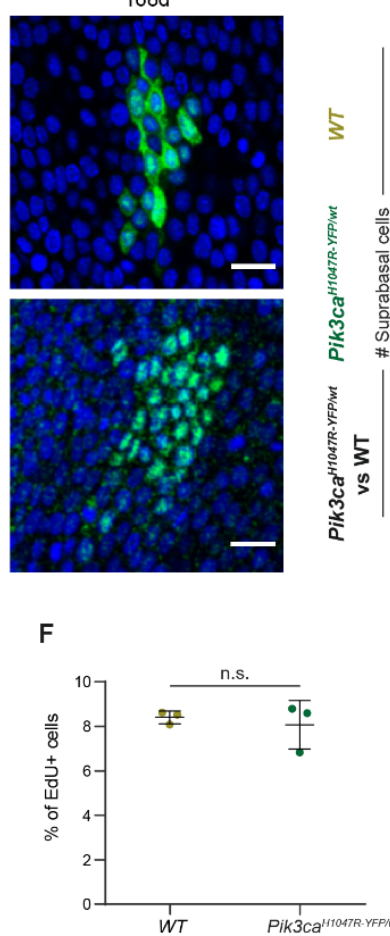

D
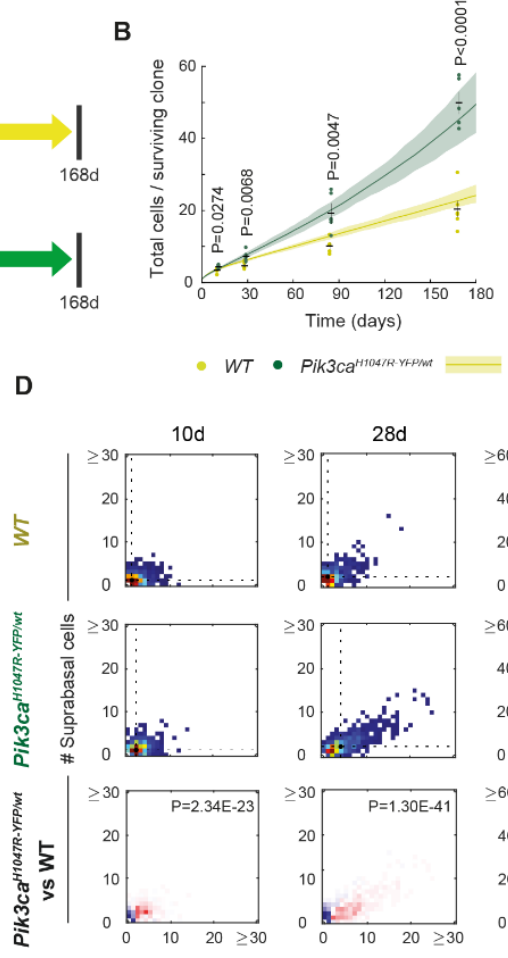

G

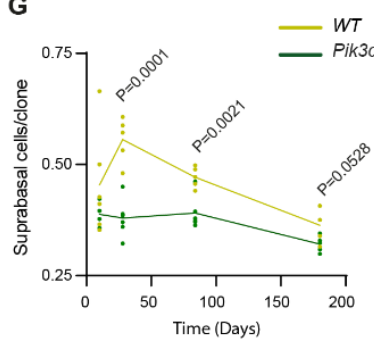

H

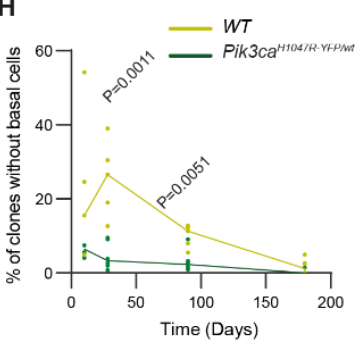

I

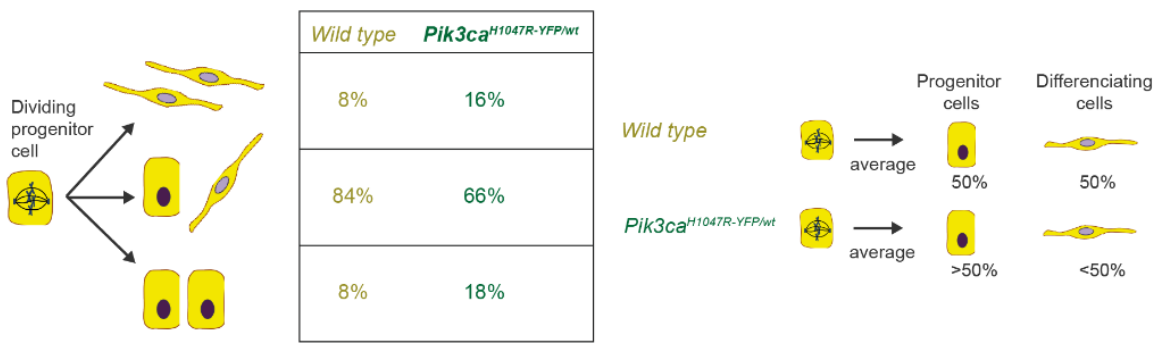

Figure 3. Lineage tracing reveals a bias in Pik3ca ${ }^{H 1047 / w t}$ cell fate favoring mutant clone growth.

(A) Experimental protocol. AhCre ${ }^{E R T} R o s a 26^{f I E Y F P / w t}$ reporter mice (Cre-RYFP) and AhCre ${ }^{E R T} P i k 3 c a^{f l H 1047 R-T 2 A-Y F P-N L S / w t}$ mice (Cre-Pik3ca ${ }^{H 1047 R-Y F P / w t}$ ) were induced with $\beta$-naphthoflavone and tamoxifen and tissue collected at the indicated time points. Wild type (WT) clones from Cre-RYFP mice and Pik3ca ${ }^{H 1047 R-Y F P / w t}$ clones from Cre-Pik3ca ${ }^{H 1047 R-Y F P / w t}$ animals were imaged and cell numbers in each clone quantified. $n=317-1522$ clones from 4-6 animals per condition (see Table S1 for values). (B) Average total cells per clone (basal layer plus first suprabasal layer cells, left panel) and basal cells per clone (right panel) over time, for all clones with at least one basal cell. Dots indicate the average clone size in a single mouse. Lines and shaded areas represent the best fitting model for the clone size distributions and its plausible intervals (see Supplementary Text). Mean and standard error of the mean per condition are indicated in black. Two-tailed unpaired $t$-test. (C) Top down views of confocal images of representative clones (green) for Cre-RYFP reporter mice (top panels) and Cre-Pik3ca ${ }^{H 1047 R-}$ YFP/wt mice (lower panels) 28 days and 168 days after induction. Nuclei are stained with DAPI (blue). An optical section through the basal cell layer is shown. Scale bars, $20 \mu \mathrm{m}$. (D) Heatmaps representing the 
frequency of clone sizes with the number of basal and first suprabasal cells indicated, for Cre-RYFP and Cre-Pik3ca ${ }^{H 1047 R-Y F P / w t}$ animals. Black dots and dashed lines show geometric median clone size. Lower panels show the differences between Cre-Pik3ca ${ }^{H 1047 R-Y F P / w t}$ and Cre-RYFP animals for each time point. 2D Kolmogorov-Smirnov test. (E) Basal cells (DAPI, blue) of a typical EE whole mount showing Pik3ca ${ }^{H 1047 R-Y F P / w t}$ cells (green), and EdU basal cells (red). Scale bar, $20 \mu \mathrm{m}$. (F) Percentages of $\mathrm{EdU}^{+}$, uninduced (WT) or Pik3ca ${ }^{H 1047 R-Y F P / w t}$, basal cells quantified in the same tissues one month after induction. 52,898 cells were quantified from 3 animals including 3,514 Pik3ca ${ }^{H 1047 R-Y F P / w t}$ cells. Each dot corresponds to an animal. Mean and standard deviation are shown in black. n.s., not significant (Ratio paired $t$-test). (G) Average proportion of suprabasal cells per clone over time, counting basal and first suprabasal cells from clones shown in (D). Each dot corresponds to one animal and the lines connect means. Two-tailed unpaired $t$-test. $(\mathrm{H})$ Proportion of floating clones per mouse. Each dot corresponds to one animal and the lines connect averages. Two tailed unpaired $t$-test. $\mathrm{n}=4-6$ animals per condition (see Table S1 for values). (I) Schematic illustration of WT and Pik3ca ${ }^{\text {H1047R-YFP/wt }}$ cell behavior. The model predictions for the proportions of each cell division fate for both genotypes are shown. A bias in the fate of Pik3ca ${ }^{H 1047 R-Y F P / w t}$ progenitors together with an increased proportion of symmetric divisions results in an increased production of mutant progenitor cells per average cell division in the esophageal epithelium, even with the rate of mutant cell division being the same as that of WT cells.

To test if these results were due to genetic background differences between mutant and control mouse strains, we crossed Cre-Pik3ca ${ }^{H 1047 R-Y F P / w t}$ mice with the Rosa26 $6^{\text {Confetti/wt }}$ strain (Snippert et al., 2014) (Figure S2A). This triple mutant allows to track Pik3ca ${ }^{\text {H1047R-YFP/wt }}$ expressing clones (labelled with YFP) and non-recombined Pik3ca wild type clones, both labelled with red fluorescent protein (RFP) in the same esophagus (Figures S2B and C). The results confirmed that the mutant clones expanded more rapidly than wild type clones in the same mouse (Figures S2D and E). Therefore, we conclude that Pik3ca ${ }^{H 1047 R-Y F P / w t}$ progenitors have a competitive advantage over their wild type neighbor cells in the mouse esophageal epithelium.

\section{Pik3ca ${ }^{H 1047 R}$ mutation biases progenitor cell fate towards proliferation}

There are several possible cellular mechanisms that may underpin the increased size of Pik3ca ${ }^{H 1047 R}$ mutant over neutral, wild type clones. To investigate whether altered cell cycle kinetics in the mutant cells were responsible for this effect, we induced and aged CrePik3ca ${ }^{\text {H1047R-YFP/Wt }}$ mice for 1 month. One hour before tissue collection, animals were injected with 5-ethynyl-2'-deoxyuridine (EdU), which labels keratinocytes in S phase of the cell cycle. The percentage of labelled basal cells depends on the duration of $S$ phase/cell cycle time and the proportion of basal layer cells that are progenitors. The proportion of $\mathrm{EdU}^{+}$basal cells was similar in mutant cells compared with wild type cells in the same esophagus (Figures 3E and F). These results argue that the cell-cycle time and the proportion of cycling progenitors are not substantially altered by the Pik3ca ${ }^{H 1047 R}$ mutation. 
Figure S2
A

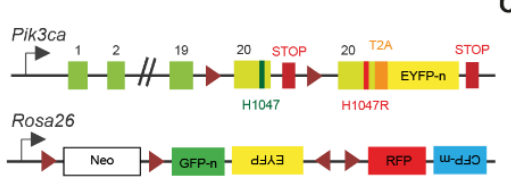

AhCre ERT $^{2}$

B

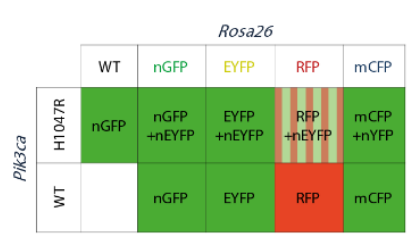

F

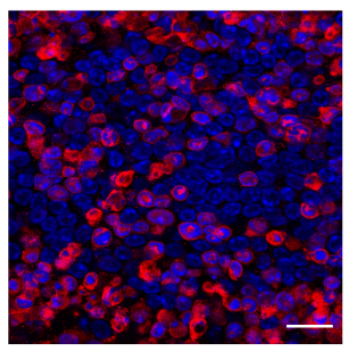

G
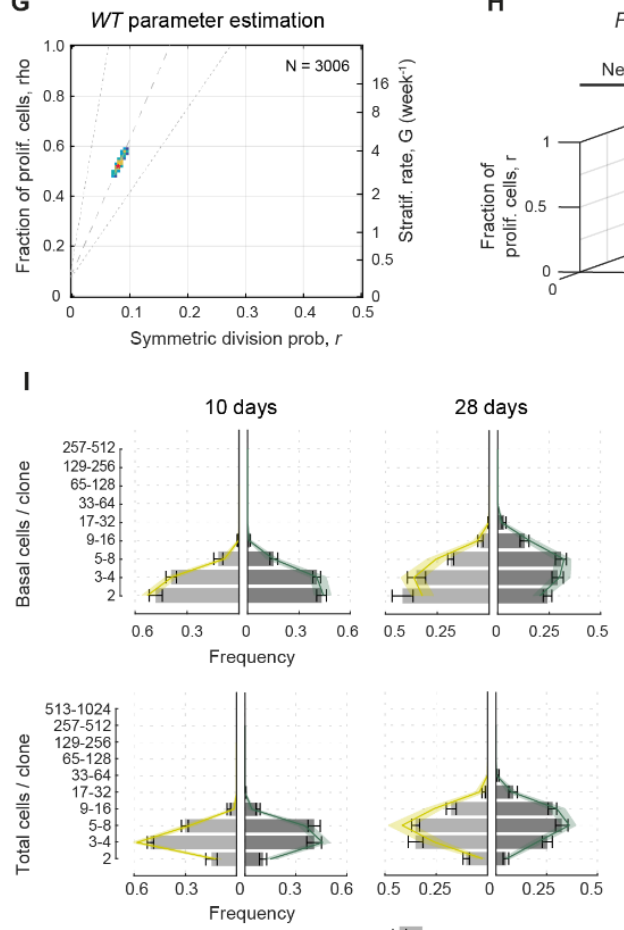

H $W T$ data (s.e.m.)

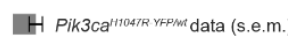

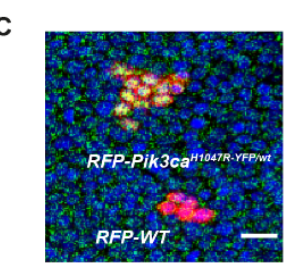

E

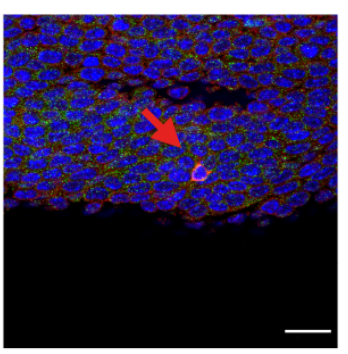

H
D

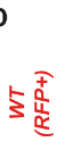
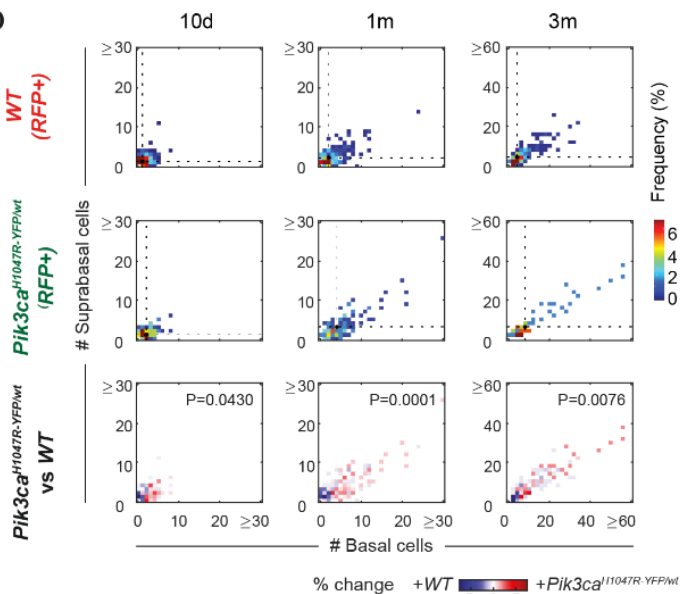
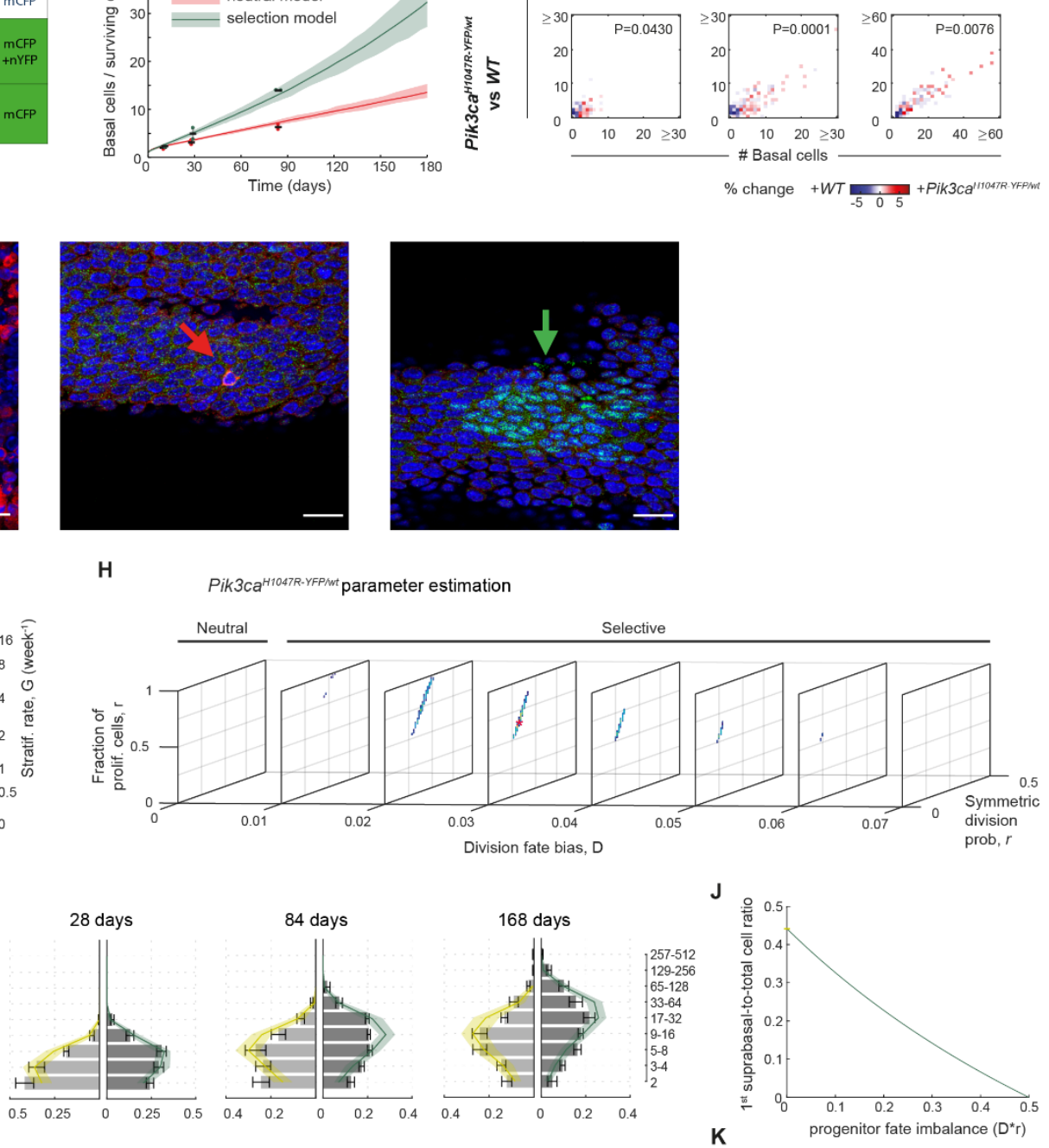

K
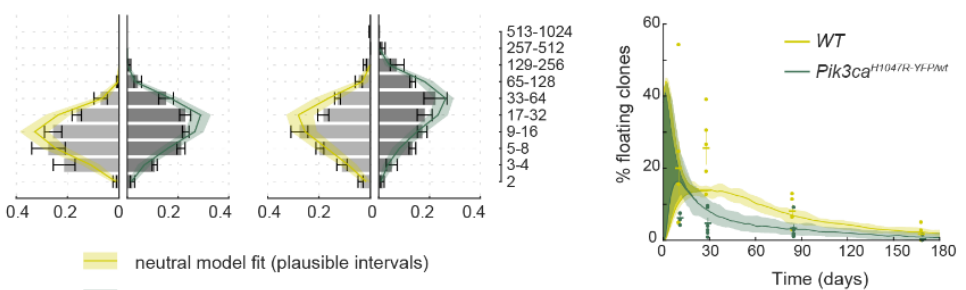

neutral model fit (plausible intervals)

= selection model fit (plausible intervals)

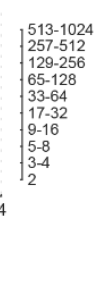

Supplementary Figure 2. Related to Figure 3. Lineage tracing in Pik3ca ${ }^{H 1047 R-}$ ${ }_{Y F P / w t}$ Rosa26 $6^{\text {confetti/wt }} A h C r e^{E R T}$ mice, apoptosis measurement and mathematical modeling for CreRYFP and Cre-Pik3ca ${ }^{H 1047 R-Y F P / w t}$ lineage tracing. (A) Multicolor lineage tracing in Pik3ca ${ }^{H 1047 R-}$ ${ }_{\text {YFP/Wt }}$ Rosa26 $6^{\text {confettiwt }} A h C r e^{E R T}$ (Cre-Pik3ca ${ }^{\text {H1047R-YFP/Wt }}$-Confetti) animals. The confetti reporter allele encodes four fluorescent proteins. After random Cre-mediated recombination induced by treating with $\beta$-naphthoflavone and tamoxifen, one fluorescent reporter is expressed by the cell and its progeny (clone). Some fluorescently labelled clones will also recombine Pik3ca locus expressing the mutation 
together with an EYFP reporter. (B) Table showing the possible color combinations obtained from (A)

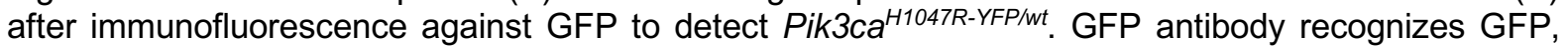
EYFP and CFP together with EYFP expressed from the Pik3ca locus. Only RFP+ clones wild type or Pik3ca ${ }^{\text {H1047R-YFP/wt }}$ can be distinguished and quantified. (C) Top down views of confocal images of an esophageal epithelium whole-mount of a Cre-Pik3ca H1047R-YFP/wt -Confetti animal 84 days after induction, showing a RFP ${ }^{+}$Pik3ca ${ }^{\text {wt } / w t}$ and a RFP ${ }^{+}$Pik3ca ${ }^{H 1047 R-Y F P / w t}$ clone. Nuclei stained with DAPI (blue). Scale bar, $20 \mu \mathrm{m}$. (D) Heatmaps representing the frequency of clone sizes, with the number of basal and first suprabasal cells indicated, of RFP ${ }^{+}$clones observed in Cre-Pik3ca ${ }^{H 1047 R-Y F P / w t}$-Confetti animals. RFP ${ }^{+}$ clones from each animal were classified into Pik3ca ${ }^{\text {wt }}$ wt and Pik3ca ${ }^{H 1047 R-Y F P / w t}$ by immunofluorescence. Black dots and dashed lines show geometric median clone size. Lower panels show the differences between Pik3ca ${ }^{\text {wtwt }}$ and Pik3ca ${ }^{\text {H1047R-YFP/wt }}$ clones. 2D Kolmogorov-Smirnov test. $\mathrm{n}=255-469$ clones in total from 2-3 animals per condition (see Table S1 for values). (E) Average basal cells per clone over time, considering all clones with at least one basal cell. Dots indicate the average clone size of a mouse. Mean and standard error of the mean per condition are indicated in black. Lines and shaded areas represent the best fitting model for the clone size distributions shown in Figure 3 and its plausible intervals (see Supplementary Text). (F) Confocal images of an esophageal epithelium basal layer whole-mount stained for activated Caspase 3 (red), GFP (Pik3ca ${ }^{\text {H1047R-YFP/wt }}$ cells, green) and DAPI (blue). Left panel shows a UV irradiated sample as a positive control, exposed to ultraviolet radiation and maintained as explant culture. Middle and right panels are images from the same tissue showing an apoptotic cell (red arrow) and a Pik3ca ${ }^{\text {H1047R-YFP/wt }}$ clone (green arrow). Scale bars, $20 \mu \mathrm{m}$. (G-H) Results of parameter inference for wild type $(\mathrm{G})$ and mutant $(\mathrm{H})$ progenitor cells. Distributions of the number of basal cells/clone were fitted (see Supplementary Text) and heatmaps show most-likely parameter values according to likelihood inference for a neutral single-progenitor model with balanced fates (wild type, G) and when extending the analysis to a single-progenitor model with imbalanced fates, displaying selection (mutant, $\mathrm{H}$ ). In the latter case, different values for the parameter of division fate bias $\Delta$ were considered ( $\Delta=0$ means neutral behavior). Red asterisk: maximum likelihood estimate (MLE). Colored regions fall within $95 \% \mathrm{Cl}$ (uncolored regions are out of bounds). (I) Distributions of wild type (light grey) and mutant (dark grey) clone sizes. Number of basal cells/clone, and number of total (basal + first suprabasal) cells/clone are displayed (top and bottom panels, respectively) (sizes grouped in powers of two). Error bars: experimental mean \pm s.e.m. Overlaid are MLE model fits (shaded areas represent $95 \%$ plausible intervals given the total number of clones counted at each time point). (J) Theoretical prediction for the effect of a progenitor fate imbalance on the relative proportion of first suprabasal cells. The first suprabasal-to-total cell ratio decreases with $\Delta^{*} r$ following a rational decay function (see Supplementary Text) (initial departure point corresponds to wild type value). (K) Proportion of floating clones (i.e. suprabasal clones having no basal attachment) over time. Yellow and green dots correspond to values in individual wild type and mutant mice, respectively (error bars: mean \pm s.e.m.). Overlaid are MLE model fits once changes in suprabasal-to-total cell ratio over time were considered (shaded areas defined as in I).

Another potential mechanism of cell competition is by promoting apoptosis of neighboring cells (de la Cova et al., 2014). However, there was a negligible level of apoptosis in wild type cells, whether adjacent to or distant from mutant clones in induced Cre- Pik3ca ${ }^{\text {H1047R-YFP/Wt }}$ animals (Figure S2F).

Finally, we investigated whether Pik3ca ${ }^{H 1047 R-Y F P / w t}$ clone behavior could be explained by altered progenitor cell fate. Even when cell division rates are similar, mutant populations could still expand by producing more progenitor than differentiating daughter cells per average cell division, as previously observed with some other genetic mutants (Alcolea et al., 2014; Murai et al., 2018; Piedrafita et al., 2020). Mathematical modeling revealed that wild type clones follow a neutral model of cell competition, as described previously, with equal proportions of proliferating and differentiating cells produced from the average cell division (Figures $\mathbf{3}$ I and 
S2G) (Doupe et al., 2012; Piedrafita et al., 2020). However, Pik3ca ${ }^{H 1047 R-Y F P / w t}$ clone dynamics is explained by a non-neutral model where mutant progenitors have altered division outcome probabilities (Supplementary text). There is a fate bias towards proliferation, so that, over the mutant progenitor population, the average cell division generates an excess of proliferating over differentiating progeny, explaining the clonal growth advantage over wild type cells (Figure $\mathbf{3 l}$ and $\mathbf{S 2 H}$ ). This simple model fits both the observed basal cell and total (basal plus suprabasal) clone size distributions and averages (Figures 3B and S2I). The model also predicts that a progenitor fate imbalance should result in a decreased proportion of suprabasal cells per clone (Figure S2J) and a reduction in the number of fully differentiated clones lacking any basal cells (floating clones) in the mutant (Figure S2K, Supplementary Text). These predictions also fit with experimental data since both the proportion of suprabasal cells per clone and floating clones were significantly reduced in the mutant (Figures $\mathbf{3 G}$ and $\mathbf{H}$ ).

Therefore, Pik3ca ${ }^{\text {H1047R-YFP/wt }}$ keratinocytes show a bias in basal cell fate towards the generation of more progenitor than differentiating daughters, resulting in mutant cells having a competitive advantage over wild type cells in the esophageal epithelium (Figure 3l, Supplementary video)

\section{Pik3ca ${ }^{\text {H1047R-YFP/wt }}$ mutant cell fitness depends on the level of PI3K pathway activation in neighboring wild type cells}

To further investigate the basis of the mutant cell advantage over wild type cells we used a 3D stratified primary culture system suitable for long-term cell competition studies. We generated primary esophageal keratinocyte cultures from Rosa ${ }^{\text {RYFP/RYFP }}$ (Pik3ca ${ }^{\text {wtwt }}$, henceforth referred to as WT-RYFP) and Pik3ca ${ }^{\text {H1047R-YFP/Wt }}$ mice, and induced recombination by infecting these cultures with adenovirus encoding Cre recombinase (Figures S3A and B). Due to the much higher level of YFP reporter expression in WT-RYFP compared with Pik3ca ${ }^{H 1047 R-Y F P / W t}$ cells, they can be easily identified by flow cytometry (Figure S3C). 
bioRxiv preprint doi: https://doi.org/10.1101/2021.05.28.446104; this version posted May 28, 2021. The copyright holder for this preprint (which was not certified by peer review) is the author/funder, who has granted bioRxiv a license to display the preprint in perpetuity. It is made available under aCC-BY 4.0 International license.

Figure S3

A
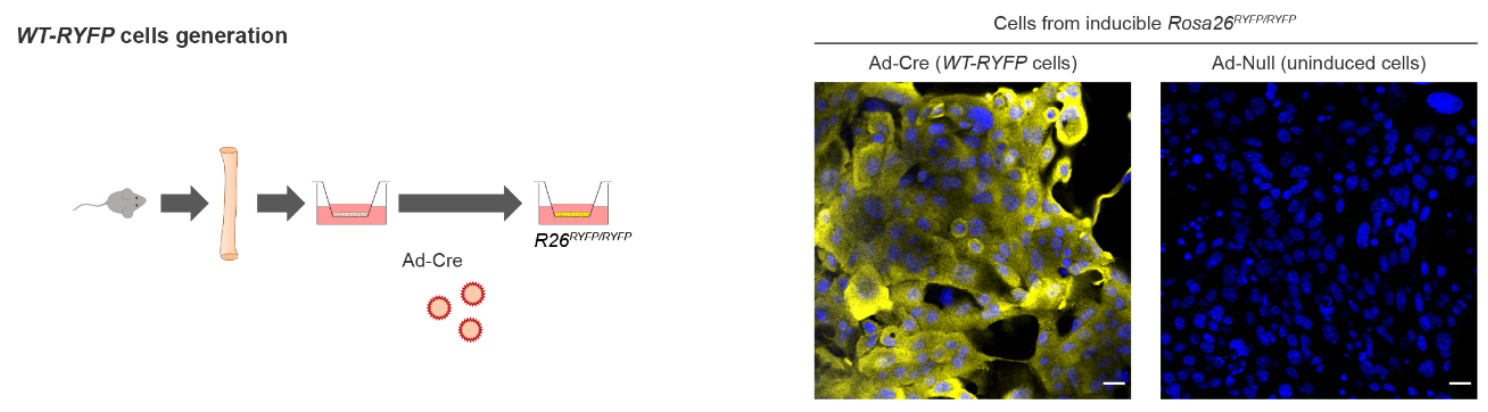

B

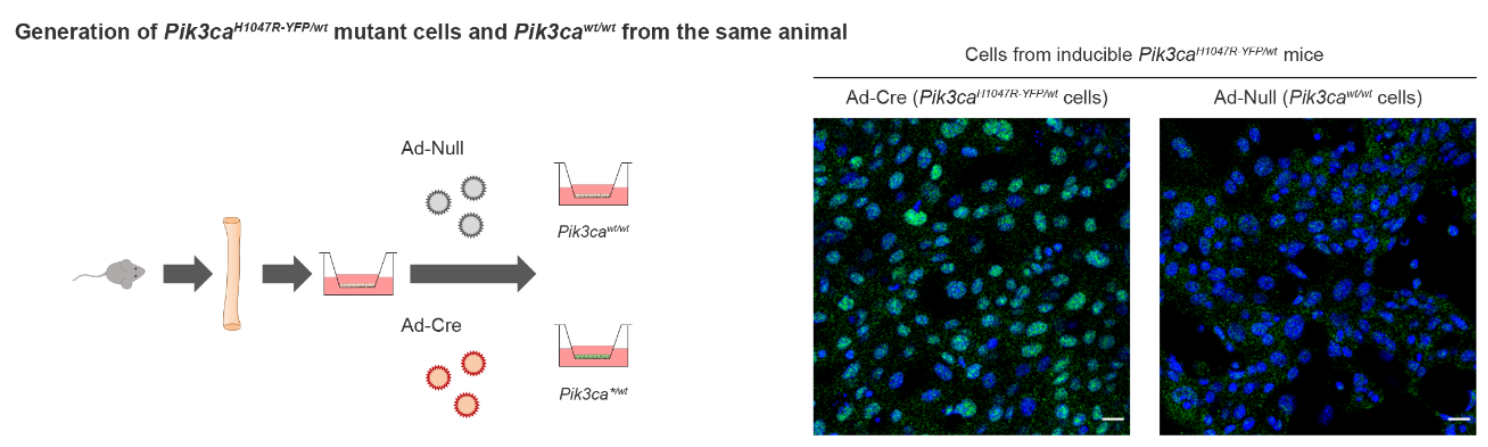

C

Cell competition experiment setup

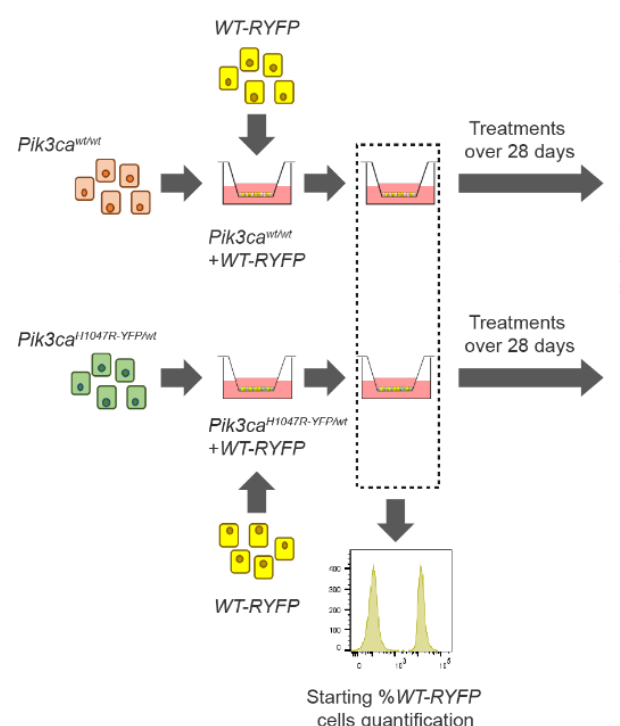

E

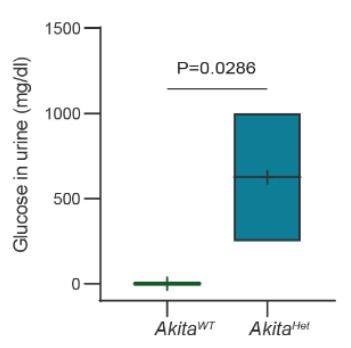

D
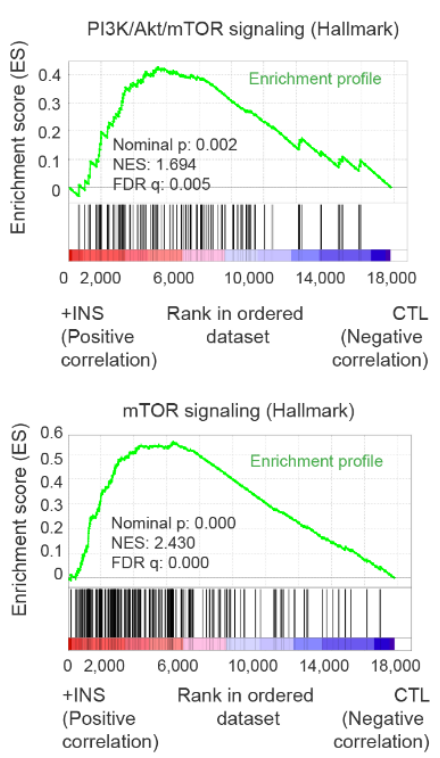

Supplementary Figure 3. Related to Figure 4. Set up for cell competition experiments. (A-B) Generation of fully induced primary WT-RYFP $(\mathrm{A})$ and $P i k 3 c a^{H 1047 R-Y F P / w t}(\mathrm{~B})$ esophageal keratinocytes. Primary esophageal keratinocytes were isolated from uninduced Rosa26 $6^{R Y F P / R Y F P}$ animals $(A)$ or uninduced Pik3ca ${ }^{H 1047 R-Y F P / w t}$ animals (B). Cells were incubated either with Cre-expressing adenovirus (Ad-Cre) or null adenovirus (Ad-Null). Right panels show a representative image of an immunofluorescence against YFP (green) and DAPI (blue) of Ad-Cre or Ad-Null treated cultures. Scale 
bars, $20 \mu \mathrm{m}$. (C) Cell competition experimental protocol. Pik3ca ${ }^{\text {H1047R-YFP/wt }}$ and Pik3cawt/wt primary keratinocytes obtained in (B) were mixed with WT-RYFP cells obtained in (A). Upon reaching confluence, cultures were changed to minimal FAD medium or the specified treatment. Cells were collected at the start and end of the treatment and the proportion of WT-RYFP cells was quantified by flow cytometry. The proportion of WT-RYFP after treatment was normalized to the initial WT-RYFP cell proportion. (D) GSEA histograms of PI3K/Akt/mTOR and mTOR signalling Hallmark gene sets comparing RNA-seq data from control (CTL) and $5 \mu \mathrm{g} / \mathrm{ml}$ insulin (+INS) treated Pik3ca ${ }^{H 1047 R-Y F P / w t}$ cells from the same animals. The nominal $p$-value, the normalized enrichment score (NES) and the false discovery rate (FDR) q-value are indicated. $n=4$ independent replicates per condition from one animal each. (E) Glucose levels in urine of the mice used in Figure $4 \mathrm{I}-\mathrm{K}$ at the day of induction. Two-tailed Mann-Whitney test. $\mathrm{n}=4$ animals per condition.

For the cell competition studies, we mixed WT-RYFP keratinocytes with either induced or uninduced Pik3ca ${ }^{H 1047 R-Y F P / W t}$ cells and followed how the proportion of WT-RYFP cells changed over time (Figures 4A and S3C). We first established that when uninduced Pik3ca ${ }^{H 1047 R-Y F P / w t}$ cells were mixed with WT-RYFP cells, the proportion of cells of both strains remained constant over time, meaning their competition is neutral (Figure 4B upper panels and C). In addition, the ratio of suprabasal:basal cells was similar in both subpopulations after 14 days of cell competition (Figure 4D). However, when induced Pik3ca ${ }^{H 1047 R-Y F P / W t}$ and WT-RYFP cells were co-cultured, the Pik3ca ${ }^{\text {H1047R-YFP/wt }}$ cells almost completely took over the culture within 28 days (Figure 4B lower panels and $\mathrm{C}$ ). Moreover, the suprabasal:basal ratio of induced Pik3ca ${ }^{\text {H1047R-YFP/wt }}$ cells was lower than for the WT-RYFP cells in the same culture (Figure 4D). We conclude that Pik3ca ${ }^{H 1047 R-Y F P / w t}$ mutant cells retain their competitive advantage over wild type cells in vitro.

If the advantage of Pik3ca ${ }^{\text {H1047R-YFP/wt }}$ mutation depends on over-activation of the PI3K/mTOR axis, we reasoned that activating this pathway in wild type cells would decrease the fitness advantage of the mutant cells, by levelling out the signaling differences between the two genotypes. High concentrations of insulin activate PI3K/mTOR via the insulin and IGF1 receptors (Boucher et al., 2010). Thus, in a mixed culture both wild type and mutant cells might experience strong PI3K/mTOR activation. We treated mixed cultures with a dose of insulin that induced transcriptional changes consistent with PI3K/mTOR pathway activation in both wild type and mutant cells (Figure 4E and S3D). The advantage of the Pik3ca ${ }^{\text {H1047R-YFP/wt }}$ over Pik3ca ${ }^{\text {wt/wt }}$ cells was substantially reduced by insulin treatment (Figure 4F and G). In addition, insulin treatment lowered the ratio of suprabasal:basal compartment in wild type cells close to that seen when competing with uninduced Pik3ca ${ }^{\text {wt/wt }}$ cells (Figure 4D). We conclude that differential activation of the PI3K pathway underpins the competitive advantage of mutant over wild type cells (Figure $\mathbf{4 H}$ ). 
Figure 4

A

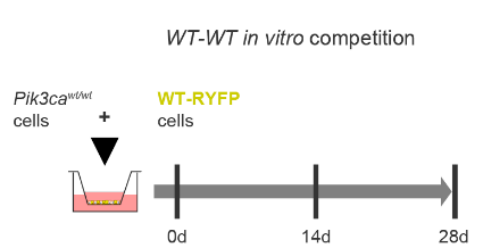

WT-Pik3ca H1047R-YFP/Wt in vitro competition

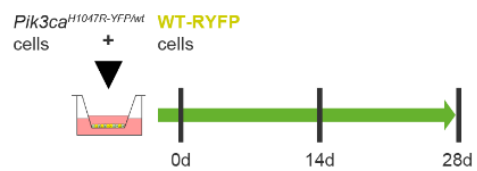

D

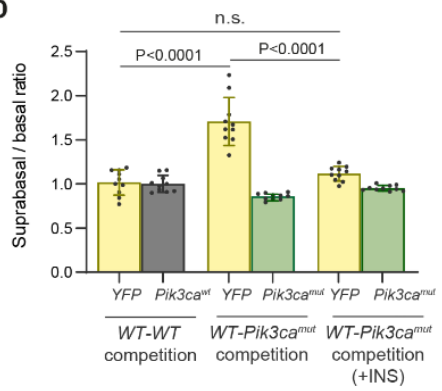

$\mathbf{F}$

WT-Pik3ca ${ }^{\text {H1047R-YFPPWt }}$ in vitro competition (28d)

CTL
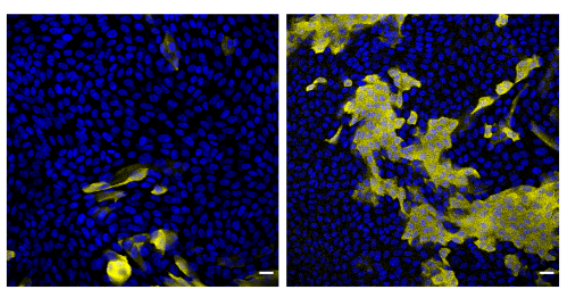

I

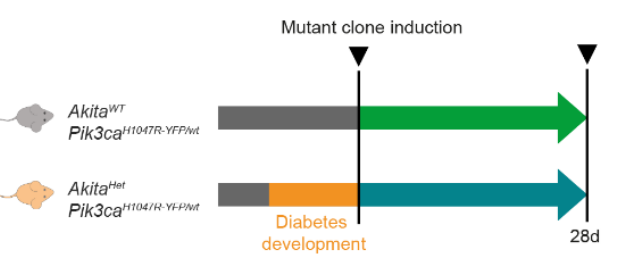

B

Od
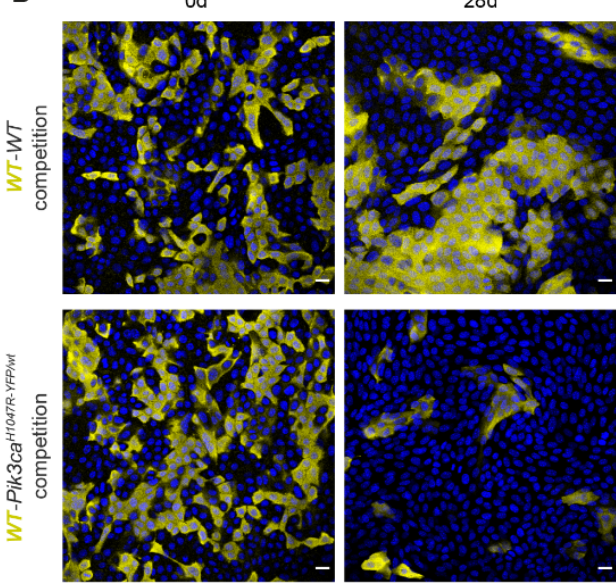

E
C

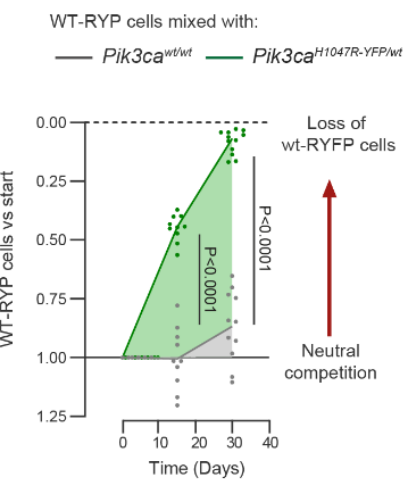

G

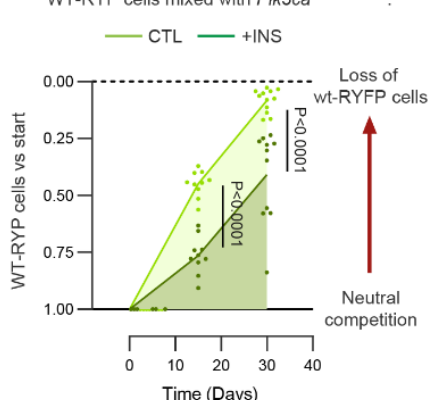

J

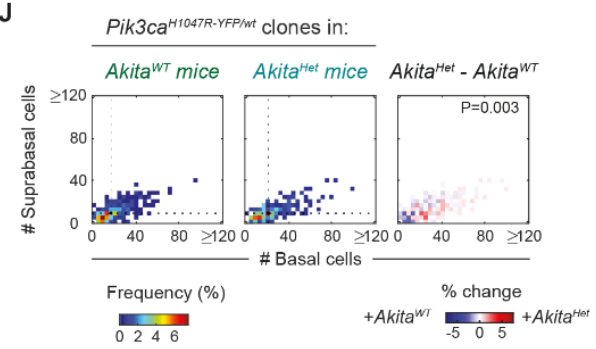

H

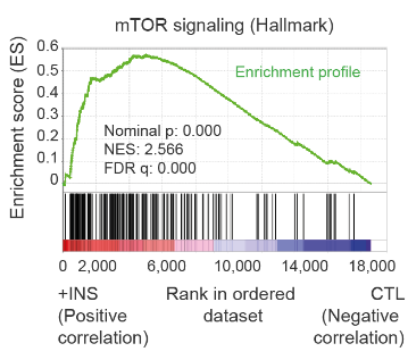

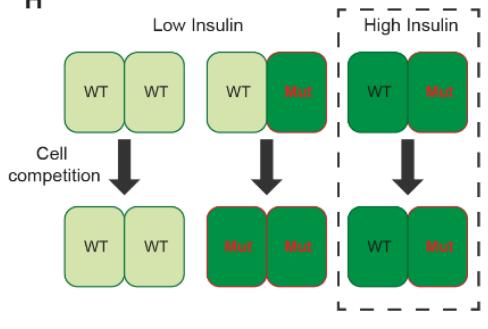

Low PI3K pathway $\quad$ High PI3K pathway
K

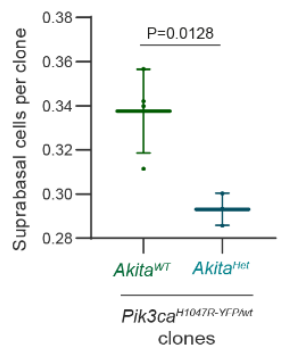

Figure 4. Differential PI3K pathway activation modulates the competitive advantage of Pik3ca ${ }^{H 1047 R-Y F P / w t}$ cells. (A) Experimental protocol for in vitro competition. In vitro induced Rosa26 $6^{\text {fYFPFIIYFP }}$ cells (WT-RYFP) were mixed with in vitro induced Pik3ca ${ }^{\text {H1047R-YFP/wt }}$ cells or uninduced $P i k 3 c a^{\text {wtiwt }}$ cells from the same animals (see Figure S3). Once a confluent culture was achieved, cells were kept for 28 days in culture with minimal FAD medium, or the specified treatment, for the duration of the experiment. Samples were collected at the start of the treatment and at 14 and 28 days. (B) Confocal z stack image representative of the specified mixed culture and time of treatment. An optical section through the basal cell layer is shown. YFP immunofluorescence (yellow), nuclei are stained with DAPI (blue). Scale bar, $20 \mu \mathrm{m}$. (C) Quantification by flow cytometry of the proportion of WT-RYFP cells versus the start of the experiment at the specified time points. Each dot represents a primary culture 
from a different animal. $\mathrm{n}=10-11$ primary cultures from individual animals per condition. Two-tailed ratio paired $t$-test. (D) Proportion of suprabasal versus basal cells of each subpopulation in mixed cultures 14 days after the start of the experiment. +INS indicate cultures treated with $5 \mu \mathrm{g} / \mathrm{ml}$ insulin. $\mathrm{n}=10$ primary cultures from individual animals per condition. n.s., not significant. Two-tailed unpaired $t$-test. (E) GSEA histograms of PI3K/Akt/mTOR and mTOR signalling Hallmark gene sets comparing RNAseq data from control (CTL) and $5 \mu \mathrm{g} / \mathrm{ml}$ insulin (+INS) treated wild type cells from the same animals. The nominal p-value, the normalized enrichment score (NES) and the false discovery rate (FDR) qvalue are indicated. $n=4$ independent replicates per condition from one animal each. $(F)$ Confocal $z$ stack image representative of the specified mixed culture and condition after 28 days of continuous treatment. An optical section through the basal cell layer is shown. YFP immunofluorescence (yellow), nuclei are stained with DAPI (blue). Scale bar, $20 \mu \mathrm{m}$. (G) Quantification by flow cytometry of the proportion of WT-RYFP cells mixed with Pik3ca ${ }^{H 1047 R-Y F P / w t}$ cells, at the specified time points versus the start of the experiment. Cells were treated either in minimal FAD medium or treated with $5 \mu \mathrm{g} / \mathrm{ml}$ insulin $(+I N S)$ for the duration of the experiment. Each dot represents a primary culture from an animal. $n=10-$ 11 primary cultures from individual animals per condition. Two-tailed ratio paired $t$-test. $(\mathrm{H})$ Summary of the results representing Pik3ca H1047R-YFP/wt (Mut) versus wild type (WT) competition in control (Low insulin) and insulin (High Insulin) conditions; in relation to the PI3K pathway activation in each subpopulation. (I) Experimental protocol: Cre-Pik3ca ${ }^{H 1047 R-Y F P / w t}$ mice were bred into Ins2 ${ }^{A k i t a / w t}$ (Akita Het)

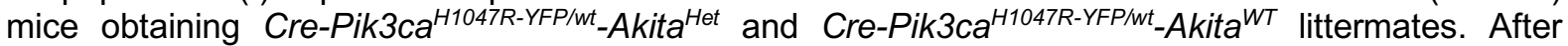
diabetes development in Akita ${ }^{\text {Het }}$ mice, they were induced with $\beta$-naphthoflavone and tamoxifen and collected after 28 days. $(\mathrm{J})$ Heatmaps representing the frequency of Pik3ca ${ }^{H 1047 R-Y F P / W t}$ clone sizes, with the number of basal and first suprabasal cells indicated, observed in Cre-Pik3ca ${ }^{\text {H1047R-YFP/Wt_A }}$ Akita ${ }^{\text {Het }}$ and Cre-Pik3ca H1047R-YFP/wt_AkitaWT littermates (left panels). Heatmap showing the differences in Pik3ca H1047R-YFP/wt clone sizes between Cre-Pik3caH1047R-YFP/wt_AkitaHet and Cre-Pik3caH1047R-YFP/wt_ Akita $^{W T}$. $\mathrm{n}=257$ and 402 clones respectively, from 4 animals per condition (see Table $\mathrm{S} 1$ for numbers). 2D Kolmogorov-Smirnov test. (K) Average proportion of suprabasal cells in Pik3ca H1047R-YFP/wt clones in Cre-Pik3ca ${ }^{\text {H1047R-YFP/wt_A }}$ kita ${ }^{\text {Het }}$ and Cre-Pik3ca ${ }^{H 1047 R-Y F P / W t}$-Akita ${ }^{W T}$ littermates. Each dot corresponds to one animal. Bars show mean and standard deviation. Two-tailed unpaired $t$-test.

These results suggest that insulin levels may alter the competitiveness of Pik3ca ${ }^{\text {H1047R-YFP/wt }}$ clones in vivo. To explore this, we turned to the Akita mouse model of type-1 diabetes, which harbors a mutation in the insulin-2 gene that results in reduced circulating insulin levels as mice age (Oyadomari et al., 2002; Yoshioka et al., 1997). We bred Cre-Pik3ca ${ }^{\text {H1047R-YFP/Wt }}$ mice onto an Akita $^{\text {Het }}$ (diabetic) or Akita ${ }^{\text {wt }}$ (non-diabetic) background. Clonal recombination was induced after the onset of the diabetes in Akita ${ }^{\text {Het }}$ mice (Figure S3E) and clones analyzed one month after induction (Figure 4I). Pik3ca ${ }^{H 1047 R-Y F P / w t}$ clones showed a reduced proportion of differentiated cells per clone in the diabetic background compared to non-diabetic littermates (Figures 4J and K), indicating that the fitness Pik3ca ${ }^{\text {H1047R-YFP/Wt }}$ mutant relative to wild type cells is higher when insulin levels in blood are low. Therefore, both in vitro and in vivo, insulin levels modulate Pik3ca ${ }^{H 1047 R-Y F P / w t}$ cell competition with wild type cells.

\section{Pik3ca ${ }^{H 1047 R}$ mutation activates HIF1a and aerobic glycolysis}

The results above argue that mutant cells may have a differential activation of the pathways downstream of PI3K to gain their competitive advantage over wild type cells. To investigate this, we compared the gene expression of induced and uninduced Pik3ca ${ }^{\text {H1047R-YFP/wt }}$ cultures generated from the same mice. RNA sequencing revealed 301 upregulated and 195 downregulated transcripts (adjusted $p$-value $<0.05$ ) in the mutant cells (Figures 5A and B). 
$47 \%$ of the upregulated genes (transcripts with an adjusted $p$-value $<0.01$ ) were known or predicted direct targets of the HIF1a transcription factor (Figure 5C), a downstream effector of the PI3K/mTOR pathway (Denko, 2008; Rohwer et al., 2019; Xie et al., 2019). Consistent with activation of the PI3K/mTOR/HIF1 axis by the Pik3ca ${ }^{H 1047 R}$ mutation, GSEA and KEGG pathway analysis showed an enrichment of the Hypoxia gene set and the HIF1a signaling pathway (Figures 5D and E). HIF1 $\alpha$ switches cell metabolism from mitochondrial oxidative phosphorylation towards aerobic glycolysis, the metabolic conversion of glucose to lactate in the presence of oxygen to produce energy (Denko, 2008). Consistent with this function of HIF1 $\alpha$, gene expression analysis showed a significant upregulation of genes encoding for all glycolysis pathway enzymes in Pik3ca ${ }^{H 1047 R}$ cells (Figures 5E, F and G). In the mutant cells, the expression of HIF1 $\alpha$ target genes that promote a metabolic switch to aerobic glycolysis (Higd1a, Bhlhe40, Bnip3, Pdk1, Ndufa4l2 and Pfkbf3) (Ameri et al., 2015; Chang et al., 2019; Kim et al., 2006; Rikka et al., 2011; Tello et al., 2011; Yi et al., 2019) and genes that promote the export of lactate and protons to reduce the intracellular acidification derived from a glycolytic metabolism, was also increased (Dovmark et al., 2017; Mboge et al., 2018) (Figures $\mathbf{5 F}$ and $\mathbf{G}$ ). To confirm the glycolytic switch in mutant cells we used high resolution respirometry which allows the measurement of the oxygen consumption rate (OCR, proportional to mitochondrial oxidation) and extracellular acidification rate (ECAR, proportional to the glycolysis to lactate) (Figure $5 F$ ). The ratio of OCR and ECAR indicates whether cells are more oxidative or more glycolytic (Zhang et al., 2012). Pik3ca ${ }^{\text {H1047R-YFP/wt keratinocytes }}$ have a significantly reduced OCR/ECAR ratio, confirming a shift to aerobic glycolysis (Figure $\mathbf{5 H}$ ). We conclude that HIF1a is activated in Pik3ca ${ }^{H 1047 R-Y F P / w t}$ cells and drives a switch to aerobic glycolysis (Figure $5 \mathrm{I}$ ).

These results suggest HIF1a may be a key effector of the Pik3ca ${ }^{H 1047 R-Y F P / w t}$ cell phenotype. To test this, we treated mixed cultures of induced Pik3ca ${ }^{\text {H1047R-YFP/wt }}$ and WT-RYFP cells with the HIF1a inhibitor PX478 (Welsh et al., 2004) (Figure 5J). The advantage of mutant over wild type cells was significantly reduced in the presence of this inhibitor (Figure 5K), arguing that activation of HIF1 $\alpha$ contributes to the competitive advantage of Pik3ca ${ }^{\text {H1047R-YFP/wt }}$ mutant cells.

We showed above that treatment with a high dose of insulin reduced the mutant cell advantage by causing PI3K pathway over-activation in wild type and mutant cells (Figure 4E-G and S3D). We speculated that this treatment may act via HIF1 $\alpha$ and glycolysis activation. Transcriptional analysis showed that $82 \%$ of the genes upregulated in the Pik3ca ${ }^{H 1047 R-Y F P / w t}$ mutant cells were also induced in wild type cells upon insulin treatment (Figure S4A and B). Insulin treatment abrogated the differences in gene expression between wild type and mutant cells (Figures S4C), particularly in HIF1a targets (Figures S4D) and glycolysis-related genes (Figures 5G). 
bioRxiv preprint doi: https://doi.org/10.1101/2021.05.28.446104; this version posted May 28, 2021. The copyright holder for this preprint

(which was not certified by peer review) is the author/funder, who has granted bioRxiv a license to display the preprint in perpetuity. It is made available under aCC-BY 4.0 International license.

Consequently, the OCR/ECAR ratio was similar in mutant and wild type cells upon insulin treatment (Figure $\mathbf{5 H}$ ). These observations suggest that insulin treatment levels out the competitive imbalance between wild type and mutant cells by over-activating the $\mathrm{PI3K} / \mathrm{HIF} 1 \alpha / g l y c o l y s i s$ axis both in wild type and mutant cells, supporting the hypothesis that a switch to glycolysis accounts for the increased fitness of mutant over wild type cells.

\section{Figure 5}

A

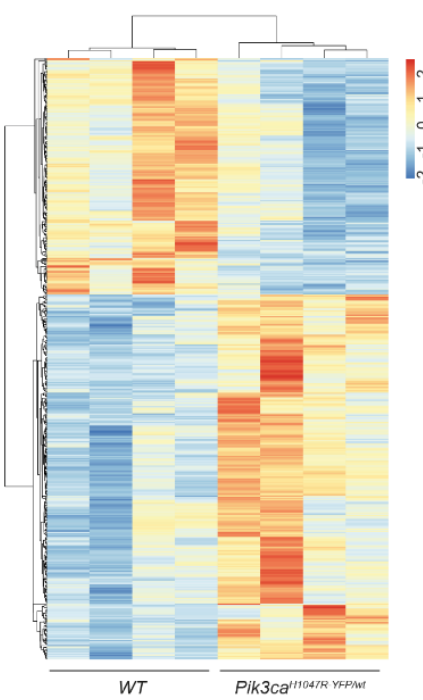

B

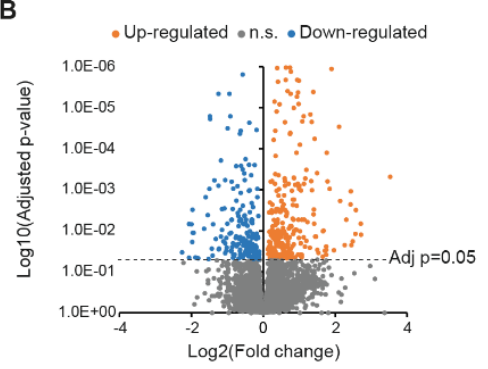

H
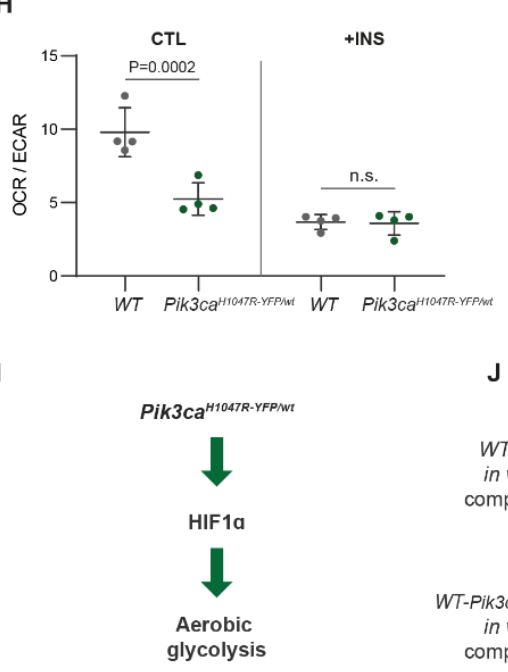

C

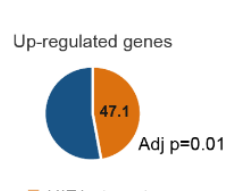

- HIF1a targets
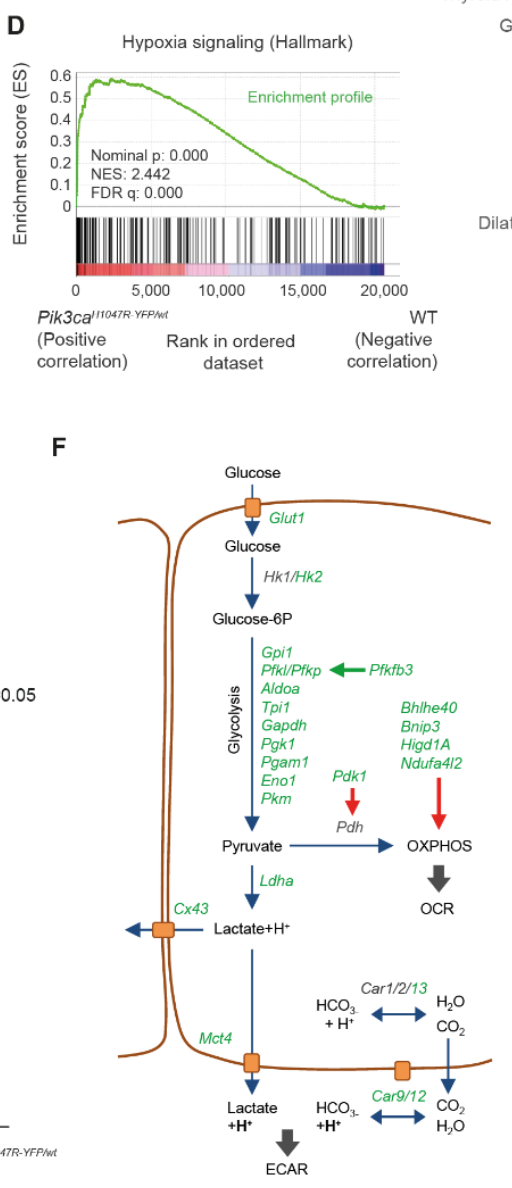

E

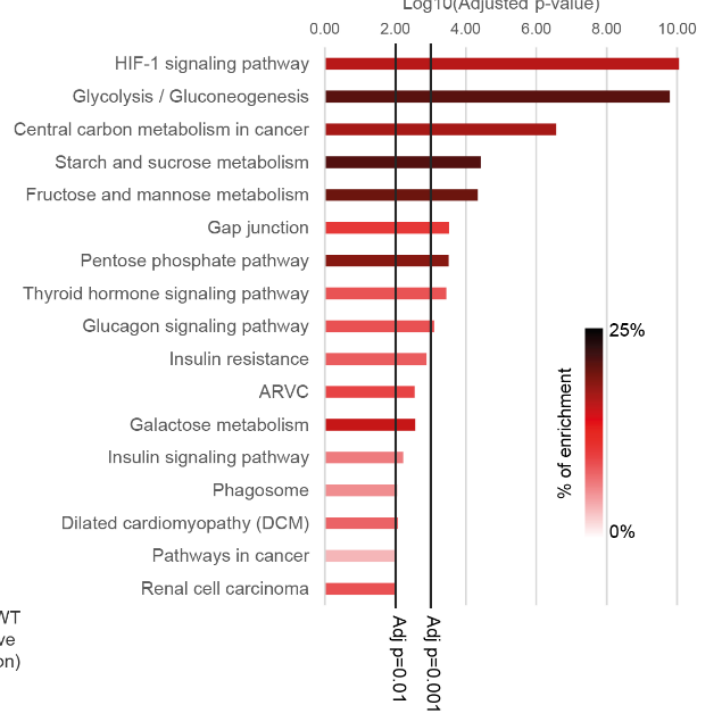

G

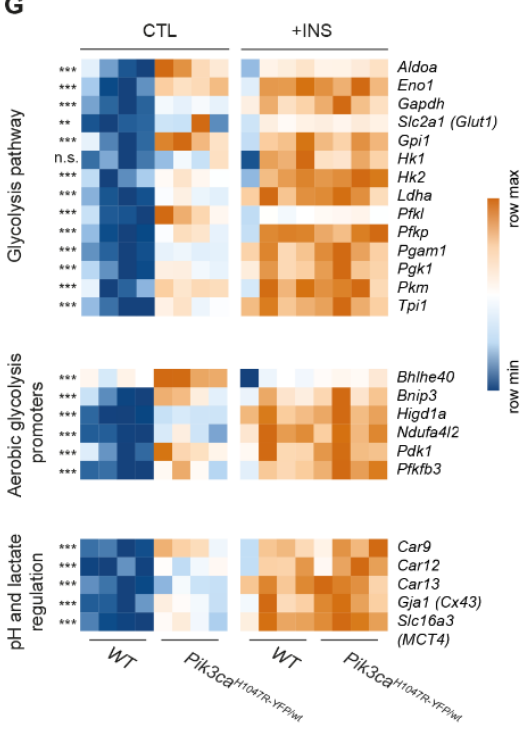

K

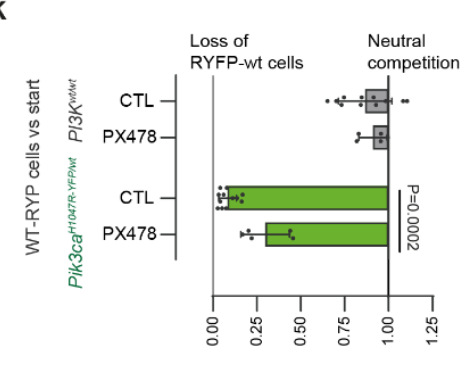


bioRxiv preprint doi: https://doi.org/10.1101/2021.05.28.446104; this version posted May 28, 2021. The copyright holder for this preprint (which was not certified by peer review) is the author/funder, who has granted bioRxiv a license to display the preprint in perpetuity. It is made available under aCC-BY 4.0 International license.

Figure 5. Pik3ca ${ }^{H 1047 R-Y F P / w t}$ primary esophageal keratinocytes activate HIF1a transcription targets and glycolysis. RNA-seq analysis of gene expression differences between in vitro induced Pik3ca ${ }^{H 1047 R-Y F P / w t}$ and uninduced Pik3ca ${ }^{\text {wt }}$ wt primary esophageal keratinocytes maintained in minimal FAD medium. Biological quadruplicates were analysed, each sample from a different animal, with an induced and uninduced pair of samples per animal. Adjusted $p$-values from Wald test corrected for multiple testing using the Benjamini and Hochberg method are shown. (A-B) Heatmap (A) and Volcano plot $(B)$ showing the up-regulated and down-regulated transcripts (adjusted $p<0.05$ ) comparing induced Pik3ca ${ }^{\text {H1047R-YFP/Wt }}$ and uninduced Pik3ca ${ }^{\text {wt/wt }}$ cells from the same animals. (C) Proportion of HIF1 $\alpha$ target genes among the genes significantly up-regulated (adjusted $\mathrm{p}<0.01$ ) in Pik3ca ${ }^{H 1047 R-Y F P / w t}$ cells. (D) GSEA histograms of the Hypoxia Hallmarks gene set, comparing Pik3ca ${ }^{\text {H1047R-YFP/wt }}$ cells versus uninduced Pik3cawtiwt cells. The nominal p-value, the normalized enrichment score (NES) and the false discovery rate (FDR) q-value are indicated. (E) KEGG pathway enrichment analysis for up-regulated genes (adjusted $p<0.01$ ) between Pik3ca ${ }^{\text {H1047R-YFP/wt }}$ cells and uninduced Pik3ca ${ }^{\text {wt } / w t}$ cells. Pathways are ordered according to its adjusted p-value and the intensity of the bar color shows its enrichment in the dataset. (F) Scheme of the glycolysis pathway showing the genes implicated and some pathway regulators together with proteins that regulate the internal $\mathrm{pH}$ homeostasis. Genes in green are upregulated in Pik3ca H1047R-YFP/wt primary keratinocytes. Green and Red arrows show activating and inhibiting processes respectively. OXPHOS: Oxidative Phosphorylation. Oxygen Consumption Ratio $(\mathrm{OCR})$ is proportional to the use of glucose through OXPHOS. Extracellular Acidification Ratio (ECAR) is produced by the protons exported to the extracellular space during aerobic glycolysis. (G) Heatmaps

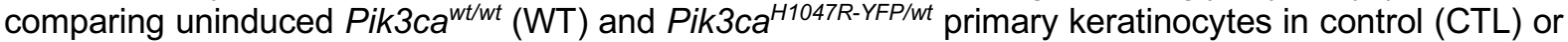
treated with $5 \mu \mathrm{g} / \mathrm{ml}$ insulin (+INS). Heatmaps show glycolysis pathway genes, genes regulating aerobic glycolysis, and genes regulating the intracellular $\mathrm{pH}$ and lactate transport. Statistical tests are performed between CTL samples. None of those genes was differentially expressed between Pik3cawtwt and Pik3ca ${ }^{H 1047 R-Y F P / w t}$ in the +INS condition. ${ }^{* * *} p<0.001$ and n.S., not significant. Wald test corrected for multiple testing using the Benjamini and Hochberg method. (H) Basal OCR to ECAR ratios of Pik3cawtwt (WT) and Pik3ca H1047R-YFP/wt primary keratinocytes in control (CTL) or treated with $5 \mu \mathrm{g} / \mathrm{ml}$ insulin (+INS). Basal OCR and ECAR were assessed using the Seahorse Extracellular Flux Analyser. Each dot represents primary cells obtained from one animal ( $n=4$ animals) using 4-5 technical replicates per animal. OCR/ECAR ratios are presented as average and standard deviation. n.s., not significant. Twotailed ratio paired $t$-test. (I) Model showing how Pik3ca ${ }^{\text {H1047R-YFP/wt }}$ cells activate HIF1a which in turn activates the aerobic glycolysis through its target genes. $(\mathrm{J})$ In vitro cell competition assay. In vitro induced Rosa26 $6_{\text {fYFP/IYYFP }}$ primary esophageal keratinocytes (WT-RYFP) were mixed with in vitro induced Pik3ca ${ }^{\text {H1047R-YFP/wt }}$ cells or uninduced controls from the same animals. Once a confluent culture is achieved, cells were treated for 28 days in culture with minimal FAD medium +/- PX-478 $(10 \mu \mathrm{M})$. Samples were collected at the start of the treatment and at 28 days and the proportion of WT-RYFP at the end of the experiment versus at the beginning was calculated. (K) Quantification by flow cytometry of the proportion of $W T-R Y F P$ cells from $(\mathrm{J})$. Each dot represents a primary culture from an animal and mean and standard deviation are shown. $n=5-11$. Two tailed unpaired $t$-test. 
bioRxiv preprint doi: https://doi.org/10.1101/2021.05.28.446104; this version posted May 28, 2021. The copyright holder for this preprint (which was not certified by peer review) is the author/funder, who has granted bioRxiv a license to display the preprint in perpetuity. It is made available under aCC-BY 4.0 International license.

Figure S4

A
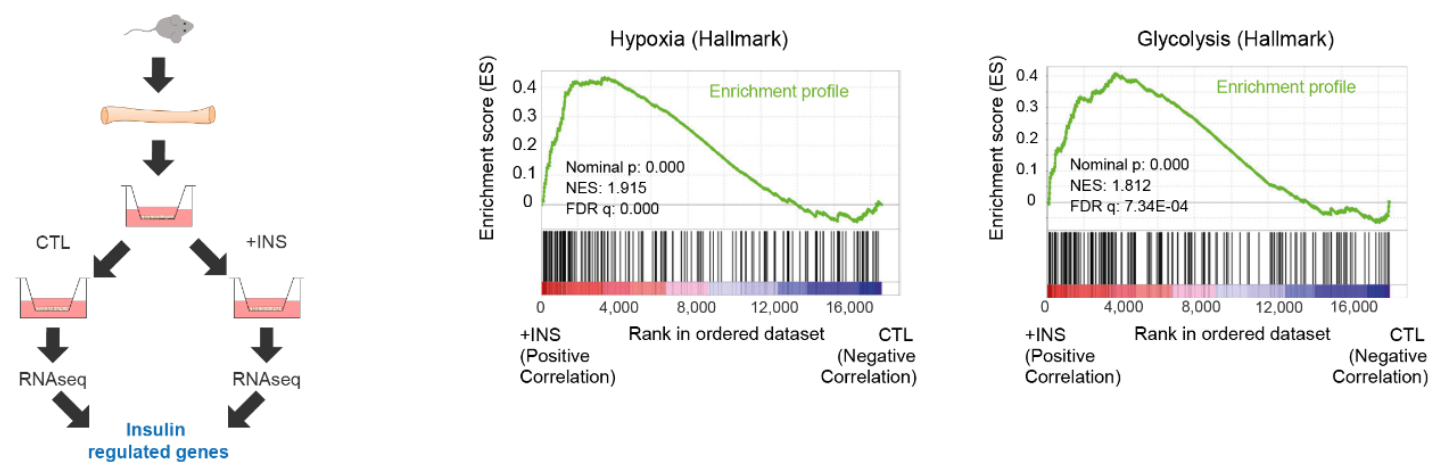

B

C
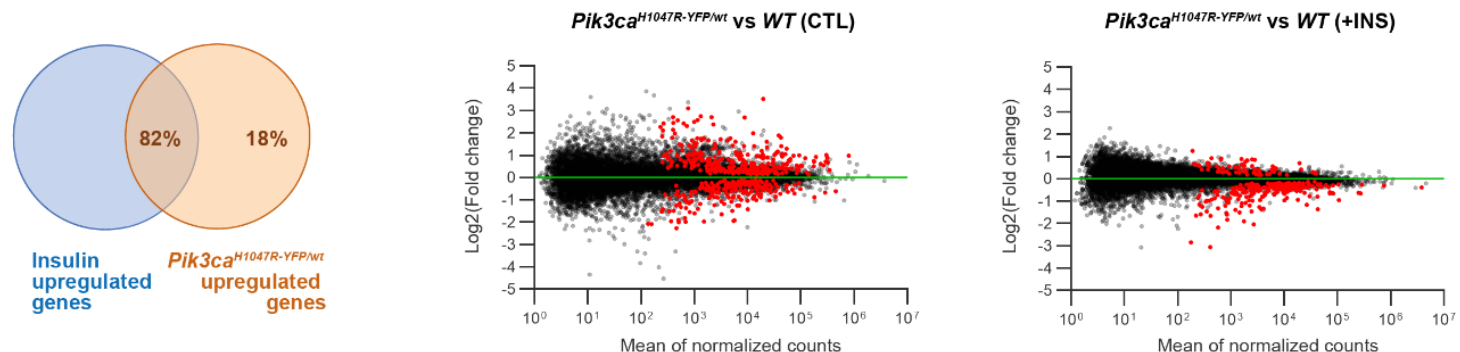

D

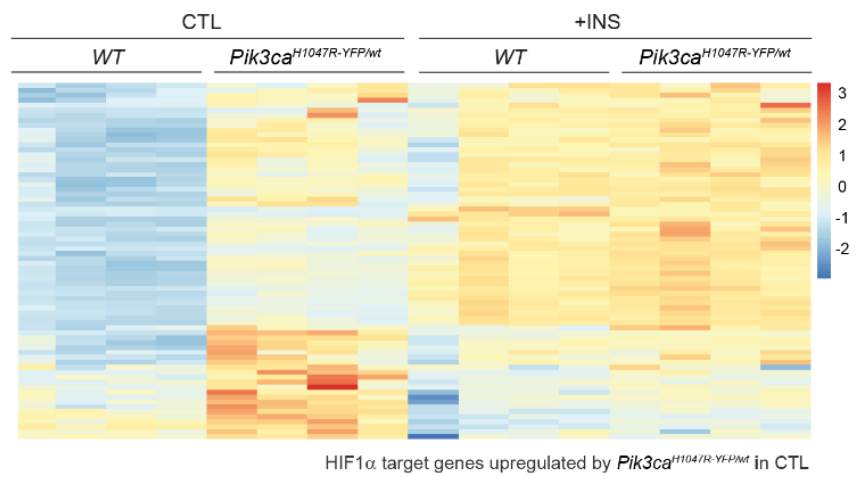

Supplementary Figure 4. Related to Figure 5. Insulin treatment activates HIF1a and Glycolysis pathways reducing most differences generated by Pik3ca ${ }^{H 1047 R}$ mutation. RNA-seq analysis of gene expression differences between in vitro induced Pik3ca ${ }^{H 1047 R-Y F P / w t}$ and uninduced Pik3cattwt primary esophageal keratinocytes maintained in minimal FAD medium (CTL) or treated with $5 \mu \mathrm{g} / \mathrm{ml}$ insulin (+INS). Biological quadruplicates were analyzed, each sample from a different animal, with an induced and uninduced pair of samples per animal. Adjusted $p$-values from Wald test corrected for multiple testing using the Benjamini and Hochberg method are shown. (A) RNA-seq analysis of culture of wild type primary esophageal keratinocytes cultured in minimal FAD medium alone (CTL) or with 5 $\mu \mathrm{g} / \mathrm{ml}$ insulin (INS). GSEA histograms of the Hypoxia and Glycolysis Hallmarks gene sets, in CTL versus INS samples. The nominal p-value, the normalized enrichment score (NES) and the false discovery rate (FDR) q-value are indicated. (B) Venn diagram showing the proportion of genes up-regulated in the Pik3ca ${ }^{H 1047 R-Y F P / w t}$ cells which are also up-regulated by the insulin treatment in wild type cells. (C) MA plots of RNA-seq data of cultures from induced Pik3ca ${ }^{H 1047 R-Y F P / w t}$ and Pik3ca ${ }^{\text {wtwt }}$ (WT) uninduced primary esophageal keratinocytes comparing CTL and +INS treatments; red indicates differentially expressed transcripts with adjusted $p<0.05$. (D) Heatmap showing the HIF1 $\alpha$ target genes significantly up-regulated (adjusted $\mathrm{p}<0.01$ ) in Pik3ca ${ }^{\text {H1047R-YFP/wt }}$ cells in the CTL condition. Heatmap shows Pik3ca ${ }^{\text {H1047R-YFP/Wt }}$ and WT uninduced primary esophageal keratinocytes comparing CTL and +INS treatments. 


\section{Metformin and DCA neutralize the competitive advantage of Pik3ca ${ }^{H 1047 R-Y F P / w t}$ clones}

To test the metabolic dependency of the competitive advantage of Pik3ca ${ }^{H 1047 R-Y F P / w t}$ cells, we investigated two complementary approaches to reduce the imbalance in glycolysis between mutant and wild type cells. We tested metformin (MET), a widely used antidiabetic agent that enhances aerobic glycolysis (Martin-Montalvo et al., 2013) and dichloroacetate (DCA), a nonselective agent that inhibits pyruvate dehydrogenase kinase-1 (PDK1), hereby forcing glycolysis-derived pyruvate to be oxidized in the mitochondria instead of being transformed into lactate, thus favoring glucose oxidation at the expense of aerobic glycolysis (Michelakis et al., 2008) (Figures 6A and S5A). Mixed cultures of Pik3ca ${ }^{\text {H1047R-YFP/wt }}$ and WT-RYFP cells were treated with either MET or DCA (Figure 6B). Both agents reduced the expansion of Pik3ca ${ }^{\text {H1047R-YFP/Wt }}$ cells in vitro suggesting that the competitive advantage of Pik3ca ${ }^{\text {H1047R-YFP/wt }}$ mutant cells is attenuated by reducing metabolic differences between mutant and wild type cells (Figures 6 C, D and E).

Finally, we determined whether MET and DCA can both inhibit clonal expansion in vivo, using Cre-Pik3ca ${ }^{\text {H1047R-YFP/Wt }}$ and Cre-RYFP mice. Animals were induced and treated with MET or DCA for one month, when clone sizes were analyzed (Figure 6F). Both MET and DCA as separate treatments reduced mutant clone size in vivo and increased the proportion of differentiated cells towards wild type levels (Figures 6G-J and S5B). These results support the hypothesis that reducing glycolytic differences between Pik3ca ${ }^{\text {H1047R-YFP/Wt }}$ mutant and neighboring wild type cells, lowers the bias towards proliferation and hence the competitive fitness of mutant progenitors (Figure 6K). 
bioRxiv preprint doi: https://doi.org/10.1101/2021.05.28.446104; this version posted May 28, 2021. The copyright holder for this preprint (which was not certified by peer review) is the author/funder, who has granted bioRxiv a license to display the preprint in perpetuity. It is made available under aCC-BY 4.0 International license.

Figure 6

A

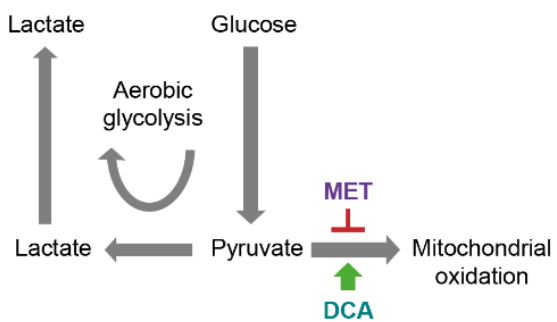

B

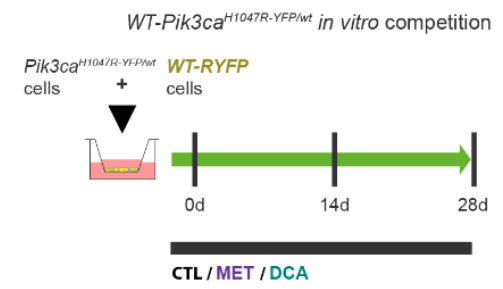

$\mathbf{F}$

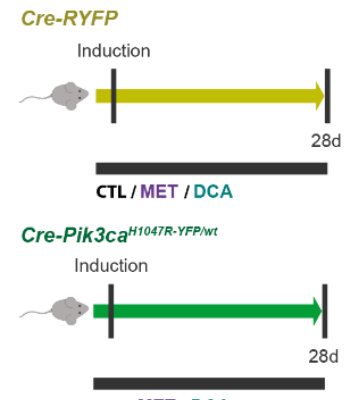

CTL/MET /DCA

G

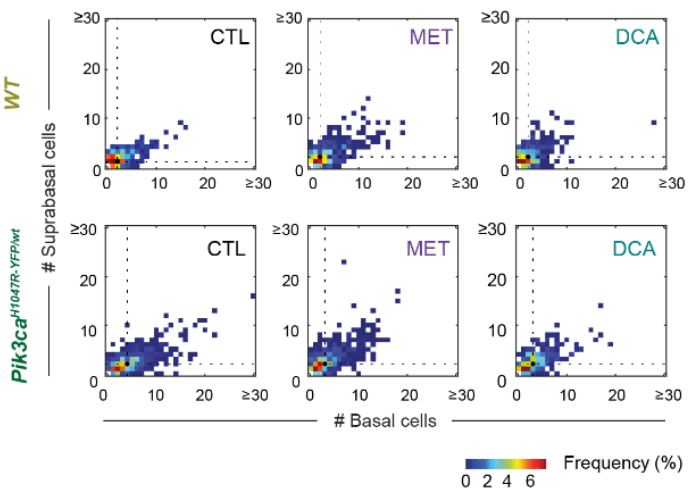

J

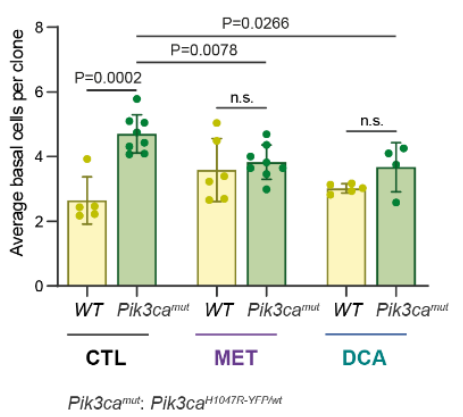

C

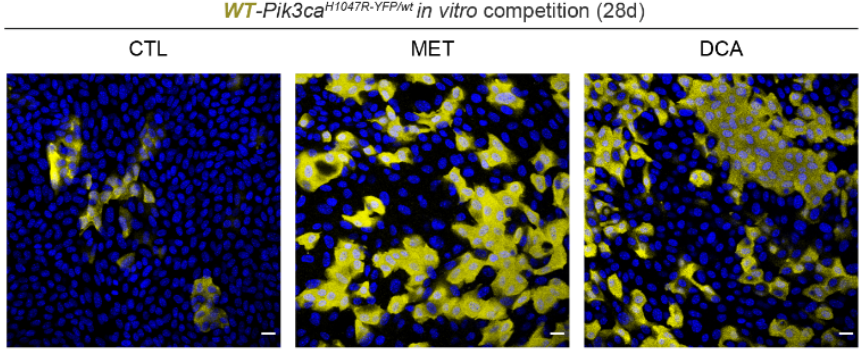

D

WT-RYP cells mixed with Pik3ca"11047R. YFPM:

- CTL $\quad+$ MET

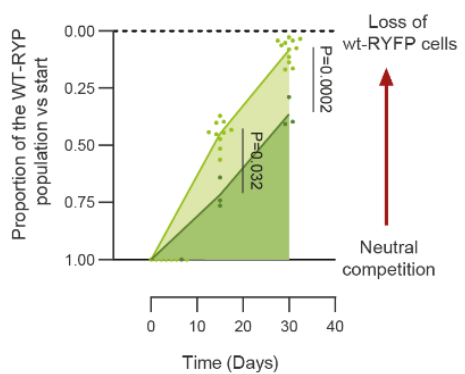

E

WT-RYP cells mixed with Pik3calit1047R YFPMt: $\longrightarrow \mathrm{CTL} \quad+\mathrm{DCA}$

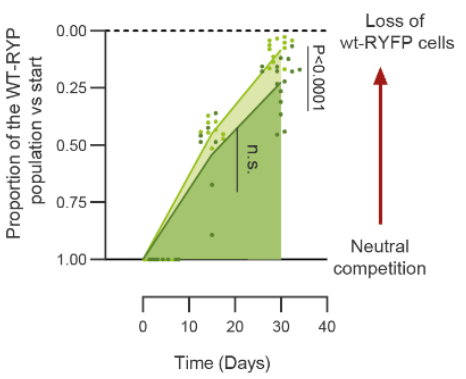

H

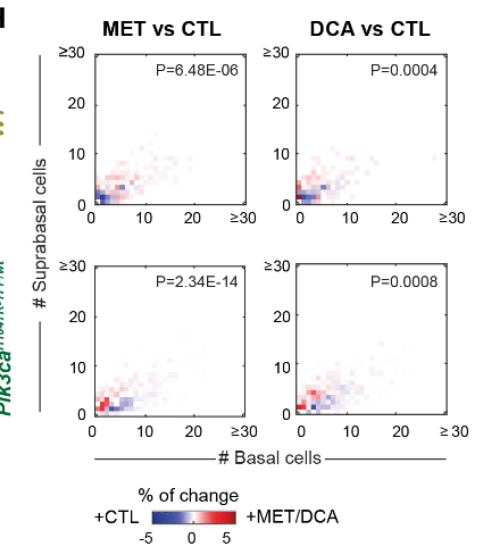

K

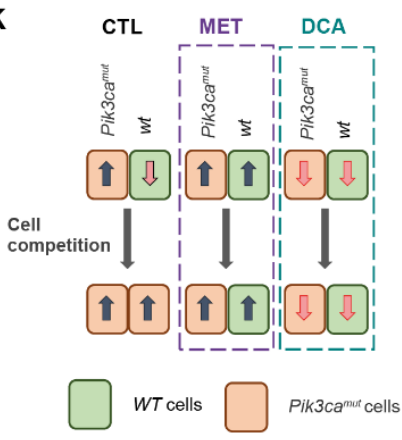

1 High / Low glycolysis

Figure 6. Metformin (MET) and DCA reduce Pik3ca ${ }^{H 1047 R-Y F P / w t}$ advantage in vitro and in vivo. (A) Scheme of energy metabolism with MET inhibiting mitochondrial oxidation (red arrow) and DCA activating glucose oxidation (green arrow). (B) In vitro cell competition assay. In vitro induced Rosa26 fIYFP/fIYFP primary esophageal keratinocytes (WT-RYFP) were mixed with in vitro induced Pik3ca ${ }^{H 1047 R-Y F P / w t}$ cells or uninduced controls from the same animals. Once a confluent culture is achieved, cells were treated for 28 days in culture with minimal FAD medium +/- MET $2.5 \mathrm{mM}$ or +/DCA $25 \mathrm{mM}$. Samples were collected at the start of the treatment and at 28 days and the proportion of WT-RYFP at the end of the experiment versus at the beginning was calculated. (C) Confocal $z$ stack image representative of the co-culture of WT-RYFP cells and Pik3ca ${ }^{H 1047 R-Y F P / w t}$ after 28 days of 
bioRxiv preprint doi: https://doi.org/10.1101/2021.05.28.446104; this version posted May 28,2021 . The copyright holder for this preprint (which was not certified by peer review) is the author/funder, who has granted bioRxiv a license to display the preprint in perpetuity. It is made available under aCC-BY 4.0 International license.

continuous treatment in minimal FAD medium or minimal FAD medium with MET $2.5 \mathrm{mM}$ or DCA 25 $\mathrm{mM}$. An optical section through the basal cell layer is shown. YFP immunofluorescence (yellow), nuclei are stained with DAPI (blue). Scale bar, $20 \mu \mathrm{m}$. (D-E) In vitro cell competition assays. Proportion of $W T$ RYFP cells, mixed either with induced Pik3ca ${ }^{H 1047 R-Y F P / W t}$ cells or uninduced controls, versus the start of the experiment, at the specified time points. Cells were treated in minimal FAD medium or minimal FAD medium with MET $2.5 \mathrm{mM}(\mathrm{B})$ or DCA $25 \mathrm{mM}(\mathrm{C})$ for the duration of the experiment. Each dot represents a primary culture from an animal. n.s., not significant. $n=3-16$ primary cultures coming from different animals. Two-tailed ratio paired $t$-test. (F) Experimental protocol. Cre-RYFP reporter mice and CrePik3ca ${ }^{H 1047 R-Y F P / w t}$ mice were induced with $\beta$-naphthoflavone and tamoxifen. They were treated with MET or DCA for the duration of the experiment and collected 28 days after induction. (G) Heatmaps showing the frequency of clone sizes with the number of basal and first suprabasal cells indicated observed in animals from $(F)$. Black dots and dashed lines indicate geometric median clone size. $n=311-$ 917 clones from 5-10 animals per condition (see Table S1 for numbers). (H) Heatmaps showing the differences between each treatment and control in Cre-RYFP (upper panels) or Cre-Pik3ca ${ }^{\text {H1047R-YFP/wt }}$ (lower panels) animals. $\mathrm{n}=311-917$ clones from 5-10 animals per condition (see Table S1 for numbers). 2D Kolmogorov-Smirnov test. (I) Average basal clone sizes for each strain and treatment from $(\mathrm{F})$, considering all clones with at least one basal cell. Dots indicate the average clone size of a mouse. Average and standard deviation per condition are indicated. $n=4-8$ animals per condition (animals with more than 50 clones). n.s., not significant. Two-tailed unpaired $t$-test. (J) Average proportion of suprabasal cells per clone for each strain and treatment from $(F)$, counting basal and first suprabasal cells. Each dot corresponds to one animal. Average and standard deviation per condition are indicated. $\mathrm{n}=4-8$ animals per condition (animals with more than 50 clones). n.s., not significant. Two-tailed unpaired $t$-test. (K) Model showing the results relating cell competition and glycolysis. Pik3ca ${ }^{H 1047 R-Y F P / w t}$ cells have increased glycolysis and an advantage over wild type cells in a control situation. Activating glycolysis with MET or reducing glycolysis with DCA; both in wild type and mutant cells, reduces the competitive advantage of Pik3ca ${ }^{\text {H1047R-YFP/wt }}$ cell.

Figure S5

A

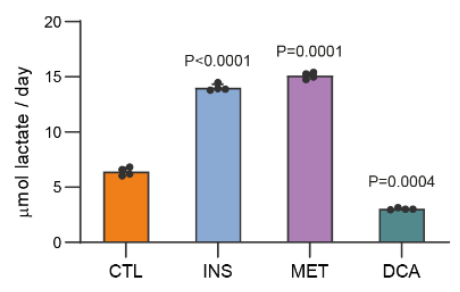

B

CTL

MET

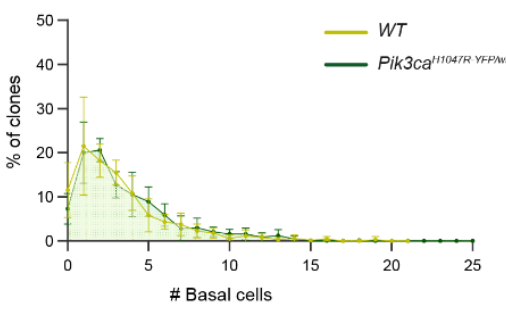

DCA

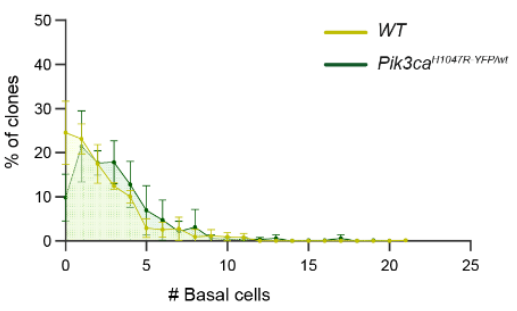

Supplementary Figure 5. Related to Figure 6. Effect of treatments in lactate secretion in vitro and effect of MET and DCA treatments on basal cell distributions in vivo. (A) Lactate secretion in wild type primary esophageal keratinocytes treated in minimal FAD medium or minimal FAD medium with $5 \mu \mathrm{g} / \mathrm{ml}$ insulin (INS), $2.5 \mathrm{mM}$ metformin (MET) or $25 \mathrm{mM}$ DCA (DCA). (B) Distribution of basal cells per clone from Cre-RYFP reporter mice and Cre-Pik3ca ${ }^{H 1047 R-Y F P / w t}$ mice after 28 days of induction and treatment. $\mathrm{n}=311-917$ clones from 5-10 animals per condition (see Table S1 for numbers). 


\section{Discussion}

The results presented here show that a subtle activation of the PI3K pathway caused by a heterozygous activating missense mutation in Pik3a is sufficient to drive clonal expansion in normal esophageal epithelium.

The cellular mechanism underpinning the competitive advantage of mutant Pik3a progenitors is a small increase in the probability of generating mutant progenitors over differentiated daughters per division, with no detectable acceleration in the cell cycle. A similar change in mutant progenitor dynamics, an increase in the proportion of proliferating versus differentiating cells per average cell division, occurs with a Notch inhibiting mutant and mutant Trp53 in the mouse esophagus and skin respectively (Alcolea et al., 2014; Murai et al., 2018). It is striking that three disparate mutations under positive selection in human esophagus all result in a similar alteration in mutant cell dynamics. These observations indicate that altering progenitor cell fate is the common mechanism hijacked by mutations in different pathways to expand in squamous epithelia. Once clones collide with others of similar fitness progenitor cell fate reverts towards balanced production of progenitor and differentiating cells (Colom et al., 2020). A small fate imbalance towards proliferating cells also occurs in high grade dysplasias and carcinomas in the mouse esophagus (Frede et al., 2016). However, while esophageal tumors have the potential to grow, competition with other mutant clones in non-transformed tissue is constrained by the limited space available for mutant clone expansion (Colom et al., 2020; Frede et al., 2016).

We confirmed that in primary esophageal keratinocytes, Pik3ca ${ }^{H 1047 R}$ expression activates glycolysis, as previously described in cell lines (Hu et al., 2016; Ilic et al., 2017; Jiang et al., 2018). However, the molecular basis of the effect of Pik3ca ${ }^{H 1047 R-Y F P / w t}$ on esophageal progenitor cell fate remains to be elucidated. Several studies of keratinocytes in the epidermis of the skin, have observed a link between the level of glycolysis and regulation of proliferation versus differentiation. For example, differentiation is increased by downregulation of glycolysis by activation of the aryl hydrocarbon receptor or inhibition of enolase (Sutter et al., 2019). Similarly, deletion of the glucose transporter, Glut1, results in decreased proliferation and increased reactive oxygen species (ROS) (Zhang et al., 2018). Elevation of ROS drives keratinocyte differentiation in both skin and esophagus (Fernandez-Antoran et al., 2019; Hamanaka et al., 2013). Finally, HIF1 $\alpha$ also promotes proliferation in human skin keratinocytes (Kim et al., 2018). Thus, our results suggest that the regulatory role for HIF1 $\alpha$ and glycolysis in keratinocyte differentiation may be a common feature of stratified epithelia. 
The expansion of Pik3ca ${ }^{H 1047 R-Y F P / w t}$ clones depends not only on the mutant cell phenotype but also the activity of the PI3K signaling axis and metabolic state of the adjacent wild type cells. Reducing the differences in PI3K/HIF1a/aerobic glycolysis axis between mutant and wild type cells, attenuates the competitive advantage of the mutant (Figure 7). These observations parallel those of mutations that activate PI3K signaling in Drosophila, which confer a fitness advantage that may be modulated by alterations in insulin exposure or metformin (Nowak et al., 2013; Sanaki et al., 2020). Nevertheless, the relationship between aerobic glycolysis and competitive fitness seems context-dependent, resulting in elimination of glycolytic mutant cells in the mouse intestine but conferring a fitness advantage in the Drosophila wing disc (Banreti and Meier, 2020; Kon et al., 2017). Likewise, strong activation of PI3K signaling by biallelic expression of Pik3ca ${ }^{H 1047 R / H 1047 R}$ in keratinocytes in the epidermis leads to their elimination by differentiation (Ying et al., 2018).

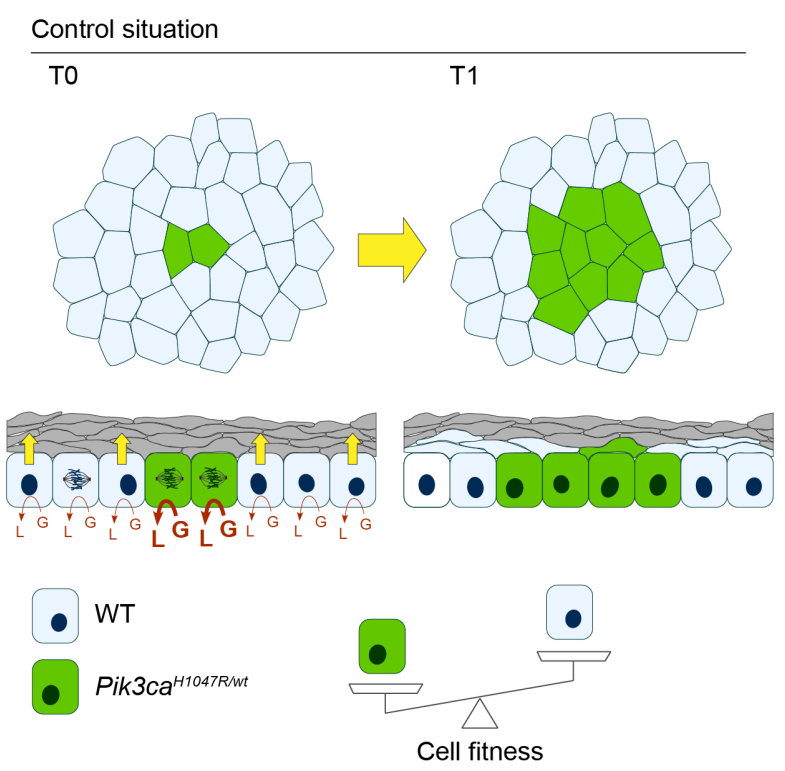

Agents activating PI3K or glycolysis in all cells
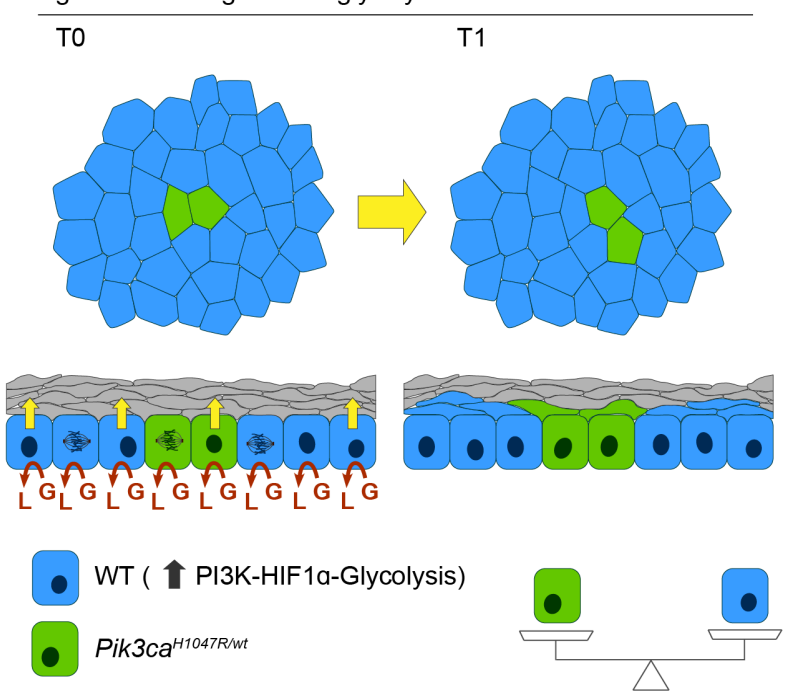

Cell fitness

Figure 7. Model for the modulation of the competitive advantage Pik3ca ${ }^{H 1047 R-Y F P / w t}$ cells by interventions activating the PI3K pathway or glycolysis. Upon mutation, Pik3ca ${ }^{H 1047 R-Y F P / w t}$ cells have increased PI3K/HIF1a/Glycolysis pathway activity over their wild type neighbors and expand in the tissue due to a reduction in cell differentiation. Interventions activating the PI3K/HIF1a/Glycolysis pathway in wild type cells or inhibiting it in mutant cells, level the cell competition and reduce Pik3ca ${ }^{\text {H1047R-YFP/Wt }}$ mutant cell expansion. L: Lactate and G: Glucose.

Our findings hint that metabolic disease states such as insulin deficiency in type 1 diabetes or treatments such as long-term metformin administration, may alter competitive selection of signaling mutants in normal tissues, by modulating the advantage of clones with mutations that activate the PI3K pathway. Such remodeling of the normal tissue landscape may impact on the risk of neoplasia and represent a potential point of intervention in cancer prevention (Bradley et al., 2018; Carstensen et al., 2016; Yu et al., 2019). Beyond cancer, it is possible that part of the aging phenotype is due to the colonization of normal tissues by mutant clones 
(Blokzijl et al., 2016; Martincorena et al., 2018). If this is so, Metformin, currently in clinical trials as an 'anti-aging' drug, may have unexpected benefits in suppressing the expansion of a subset of mutant cell clones in normal adult epithelia (Barzilai et al., 2016).

\section{Experimental Model and Subject Details}

\section{Pik3ca ${ }^{f l-H 1047 R-T 2 A-E Y F P-N L S / w t}$ generation}

Pik3ca ${ }^{f l-H 1047 R-T 2 A-E Y F P-N L S / w t}$ knock-in mice were generated by Taconic Biosciences (Hudson, NY). In the targeting vector, exon 20 (including the splice acceptor site of intron 19), the endogenous STOP sequence and the 3'UTR region of the wild type Pik3ca gene were flanked by loxP sites. A second Pik3ca exon 20 including the Pik3ca ${ }^{H 1047 R}$ mutation was introduced 3' of the distal loxP site. Between the last amino acid and the translation termination codon of the duplicated exon 20 the following sequences were inserted: a self-cleaving T2A peptide, an EYFP fused to a Nuclear Localization Signal (NLS), a STOP cassette and the 3'UTR region of the Pik3ca gene. Two positive selection markers were also introduced. A Neomycin resistance gene flanked by Frt sites was inserted $3^{\prime}$ of the $5^{\prime}$ loxP site before exon 20 . A Puromycin resistance gene flanked by F3 sites was placed downstream of the 3'UTR from the duplicated region. An additional polyadenylation signal (hGHpA: human Growth Hormone polyadenylation signal) was inserted between the 3' UTR and the distal loxP site in order to prevent downstream transcription of the mutated Pik3ca ${ }^{H 1047 R}$ exon 20 before Cre recombination. Finally, the vector included a distal thymidine kinase (Tk) gene at 3 ' end for negative selection. The targeting vector was generated using $\mathrm{BAC}$ clones from the $\mathrm{C} 57 \mathrm{BL} / 6 \mathrm{~J}$ RPCIB-731 BAC library and transfected into the Taconic Biosciences C57BL/6N Tac ES cell line. Homologous recombinant clones were isolated using double positive (NeoR and PuroR) and negative (Tk) selections. The appropriate insertion of the vector sequence was assessed by PCR. The conditional knock-in allele was obtained after in vivo Flp-mediated removal of the selection markers. This allele expresses the wild type p110 a protein. The presence of the hGHpA cassette downstream of the wild type exon 20 prevents transcription of the mutated $\mathrm{H} 1047 \mathrm{R}$ exon 20 and the EYFP before Cre recombination. The constitutive knock-in allele is obtained after Cre-mediated deletion of wild type exon 20 and the hGHpA. This allele expresses a chimeric transcript harboring the mutated $\mathrm{p} 110 \alpha^{\mathrm{H} 1047 \mathrm{R}}$ protein fused to the T2A sequence and the EYFP open reading frame including the NLS. The expected co-translational cleavage at the T2A sequences result in co-expression of the mutated $\mathrm{p} 110 \alpha^{\mathrm{H} 1047 \mathrm{R}}$ and EYFP proteins under the control of the endogenous Pik3ca promoter.

\section{Mouse experiments}


All experiments were approved by the local ethical review committees at the Wellcome Sanger Institute, and conducted according to Home Office project licenses PPL70/7543, P14FED054 and PF4639B40. Animals were maintained on a C57/BI6 genetic background, housed in individually ventilated cages and fed on standard chow. Experiments were carried out with male and female animals and no gender specific differences were observed. For lineage tracing experiments, the relevant floxed reporter lines were crossed onto the Ahcre ${ }^{E R T}$ strain in which transcription from a transgenic CYP1A1 (arylhydrocarbon receptor, Ah) promoter is normally tightly repressed until is activated by $\beta$-napthoflavone (Kemp et al., 2004). In this model tamoxifen promotes the nuclear translocation of Cre ${ }^{\text {ERT }}$ protein to mediate recombination. For lineage tracing of control clones, the Rosa26 fIYFP/wt mice which express yellow fluorescent protein (YFP) from the constitutively active Rosa 26 locus were used (Srinivas et al., 2001). To assess the mutant and wild type clone growth in the same mouse, Rosa26 fiConfetti animals were used (Snippert et al., 2010). Homozygous Ahcre ${ }^{E R T}$ Rosa26 $^{f i C o n f e t t i}$ animals were crossed onto Pik3ca ${ }^{\text {fl-H1047R-T2A-EYFP-NLSfI-H1047R-T2A-EYFP-NLS }}$ to generate

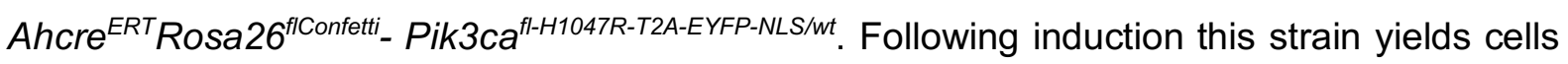
expressing just one of 4 colors of reporter from the Rosa26 locus (wild type clones), just the YFP reporter of Pik3ca mutant cells (Pik3ca ${ }^{\text {H1047R-YFP/wt }}$ clones) or both (Pik3ca ${ }^{\text {H1047R-YFP/wt_ }}$ Confetti clones) (Figure S2B). Because GFP, YFP and CFP clones are recognized by the same anti-GFP antibody used to detect the EYFP co-expressed with the mutation, only RFP expressing clones (wild type or mutant) were analyzed. Where indicated, mice were treated with a well-tolerated dose of metformin (MET, Sigma-Aldrich 317240, $2 \mathrm{mg} / \mathrm{ml}$ ) or sodium dichloroacetate (DCA, Sigma-Aldrich $347795,0.5 \mathrm{mg} / \mathrm{ml}$ ) in drinking water containing $15 \%$ Ribena to enhance palatability, for the duration of the experiment. In those cases, the results were compared to control mice treated with $15 \%$ Ribena in water. For the analysis of

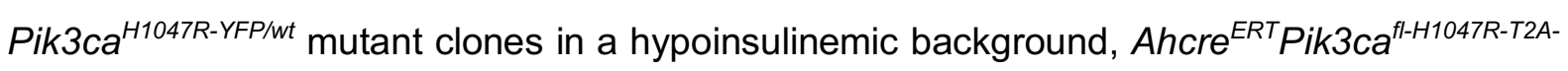
EYFP-NLS/fI-H1047R-T2A-EYFP-NLS female mice were crossed with heterozygous C57BL/6-Ins $2^{A k i t a / J}$ males $\left(\right.$ Akita $\left.^{\text {Het }}\right)$ (The Jackson Laboratory US) in order to produce Ahcre ${ }^{E R T}-$ Pik3ca ${ }^{\text {fl-H1047R-T2A- }}$ EYFP-NLS/wt Ins $2^{A k i t a / w t}$ and Ahcre ${ }^{E R T}-P i k 3 c a^{f-H 1047 R-T 2 A-E Y F P-N L S / w t}-I n s 2^{w t / w t}$ littermates. The development of diabetes phenotype was confirmed by analyzing the presence of glucose in urine, a hallmark of diabetes, at the start and end of the experiments (Figure S3D) using SURERS2/50 strips (SureScreen Diagnostics, UK) according to the manufacturer's instructions.

\section{Lineage tracing}


Low frequency expression of EYFP in the mouse esophagus was achieved by inducing transgenic animals aged 10-16 weeks with a single intraperitoneal (i.p.) of $80 \mathrm{mg} / \mathrm{kg} \beta-$

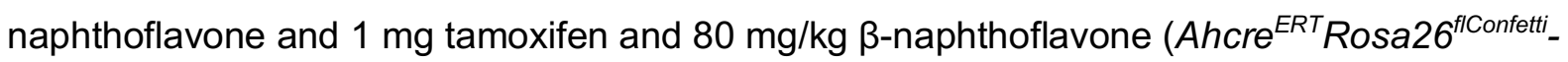
Pik3ca ${ }^{\text {fl-H1047R-T2A-EYFP-NLS/wt }}$ ) and $0.25 \mathrm{mg}$ tamoxifen (rest of mouse strains). Following induction, between three and eight mice per time point were culled and the esophagus collected. Time points analyzed include 10 days, 1, 3 and 6 months after the induction. As the expression from the endogenous Pik3ca locus is very low, immunofluorescence was necessary in order to detect the RYFP expression (Figure 2D). The total number of clones quantified for each figure can be found in Table S1. Normalized, clone-size distributions were built for each experimental condition and time point from the observed relative frequencies $f_{m, n}$ of clones of a certain size, containing $m$ basal and $n$ suprabasal cells, resulting in twodimensional histograms, (displayed as heatmaps using CloneSizeFreq_2Dheat package (https://github.com/gp10/CloneSizeFreq 2Dheat). A 2D histogram of the residuals or differences observed between conditions in the relative frequencies of each particular clone size (i.e., each cell on the grid) was generated when appropriate. 
Table S1: Related to Figures 3,S2,4 and 6

Number of quantified clones per condition and time-point

\begin{tabular}{|l|c|c|c|c|}
\hline \multirow{2}{*}{ Fig 3 } & \multicolumn{2}{|c|}{ R26 YFP/wt } & \multicolumn{2}{c|}{ Pik3caH1047R/wt } \\
\cline { 2 - 5 } & Clones quantified & Animals analysed & Clones quantified & Animals analysed \\
\hline 10 days & 1522 & 6 & 659 & 4 \\
\hline 28 days & 710 & 5 & 659 & 6 \\
\hline 84 days & 457 & 5 & 629 & 6 \\
\hline 168 days & 317 & 6 & 563 & 5 \\
\hline
\end{tabular}

\begin{tabular}{|l|c|c|c|c|}
\hline \multirow{2}{*}{ Fig S2 } & \multicolumn{2}{|c|}{ R26 $^{\text {RFP/wt }}$ Pik3cawtwt } & \multicolumn{2}{c|}{ R26 $^{\text {RFP/wt }}$ Pik3caH1047R/wt } \\
\cline { 2 - 5 } & Clones quantified & Animals analysed & Clones quantified & Animals analysed \\
\hline 10 days & 316 & 3 & 153 & 3 \\
\hline 28 days & 212 & 2 & 101 & 2 \\
\hline 84 days & 204 & 2 & 51 & 2 \\
\hline
\end{tabular}

\begin{tabular}{|c|c|c|}
\hline Fig 4 & \multicolumn{2}{|c|}{ Pik3caH1047R/wt } \\
\hline 28 days & Clones quantified & Animals analysed \\
\hline CTL & 402 & 4 \\
\hline AKITA & 257 & 4 \\
\hline
\end{tabular}

\begin{tabular}{|c|c|c|c|c|}
\hline Fig 6 & \multicolumn{2}{|c|}{ R26 YFP/wt } & \multicolumn{2}{c|}{ Pik3ca H1047R/wt } \\
\hline 28 days & Clones quantified & Animals analysed & Clones quantified & Animals analysed \\
\hline CTL & 431 & 5 & 799 & 8 \\
\hline MET & 597 & 6 & 917 & 10 \\
\hline DCA & 407 & 5 & 311 & 5 \\
\hline
\end{tabular}

\section{Quantitative analysis and mathematical modelling}

For details of quantitative analysis of wild type and Pik3ca mutant progenitor cell lineage tracing data and the dynamics of mutant cells in the suprabasal cell layers, see Supplementary Text. Code used for this analysis has been made publicly available and can be found at https://github.com/gp10/DriverClonALTfate.

\section{EdU in vivo proliferating cell analysis}

One month after lineage tracing induction, $10 \mu \mathrm{g}$ of EdU in PBS was administered by intraperitoneal injection $1 \mathrm{~h}$ before culling. Tissues were collected and stained with EdU-ClickiT kit and immunofluorescence as explained below. EdU-positive basal cells were quantified from a minimum of $10 \mathrm{z}$-stack images. 


\section{Primary keratinocyte 3D culture}

After removing the muscle layer with fine forceps, esophageal explants were placed onto a transparent ThinCert ${ }^{\mathrm{TM}}$ insert (Greiner Bio-One) with the epithelium facing upward and the submucosa stretched over the membrane, and cultured in complete FAD medium (50:50) 4.5 g/L D-Glucose, Pyruvate, L-Glutamine D-MEM (Invitrogen 11971-025): D-MEM/F12 (Invitrogen 31330-038), supplemented with $5 \mu \mathrm{g} / \mathrm{ml}$ insulin (Sigma-Aldrich I5500), $1.8 \times 10^{-4}$ $M$ adenine (Sigma-Aldrich A3159), $0.5 \mu \mathrm{g} / \mathrm{ml}$ hydrocortisone (Calbiochem 386698), $1 \times 10^{-10}$ M cholera toxin (Sigma-Aldrich C8052), $10 \mathrm{ng} / \mathrm{ml}$ Epidermal Growth Factor (EGF, PeproTech EC Ltd 100-15), 5\% fetal calf serum (PAA Laboratories A15-041), 5\% Penicillin-Streptomycin (Sigma Aldrich, P0781) and $5 \mu \mathrm{g} / \mathrm{ml}$ Apo-Transferrin (Sigma-Aldrich T2036). Explants were removed after 7 days once keratinocytes have covered half of the membrane. Media was changed every three days. Minimal FAD medium without Cholera toxin, epidermal growth factor, insulin and hydrocortisone was used from two weeks after stablishing the culture.

\section{Adenoviral infection}

To establish mouse primary keratinocyte 3D cultures from Pik3ca ${ }^{f-H 1047 R-T 2 A-E Y F P-N L S / w t}$ mice and Rosa26 fIYFPAIYFP, cells were infected with Cre-expressing adenovirus (Ad-CMV-iCre, Vectorbiolabs, \#1045 US) or Null adenovirus (Ad-CMV-Null, Vectorbiolabs, \#1300 US). Briefly, cells were incubated with adenovirus-containing medium supplemented with Polybrene (Sigma Aldrich, \# H9268) $\left(4 \mu \mathrm{g} / \mathrm{ml}\right.$ ) for $24 \mathrm{~h}$ at $37^{\circ} \mathrm{C}, 5 \% \mathrm{CO}_{2}$. Cells were washed and fresh medium was added. Infection rates were $>90 \%$.

\section{Cell competition assays}

Fully induced Rosa26 $6^{\text {fIYFPfIYFP }}$ cultures (WT-RYFP) were trypsinized and mixed, 1:1 for FACS analysis or 1:3 for microscopy, with Pik3ca ${ }^{f l-H 1047 R-T 2 A-E Y F P-N L S / w t}$ either fully induced with Creexpressing adenovirus or uninduced (infected with null adenovirus). After one week, when the cultures are fully confluent, cholera toxin, epidermal growth factor, insulin and hydrocortisone were removed from the medium and starting time point was collected and analyzed by flow cytometry. At the time points specified, cells were collected and analyzed by flow cytometry or fixed for microscopy. Where indicated, mixed cultures were treated with $5 \mu \mathrm{g} / \mathrm{ml}$ insulin (Sigma-Aldrich 15500), 10 MM PX478 (Cambridge Bioscience 10005189), 2.5 mM metformin (Sigma-Aldrich 317240) or 25 mM sodium dichloroacetate (Sigma-Aldrich 347795). 


\section{Flow cytometry}

Keratinocyte cultures were detached by incubation with $0.05 \%$ Trypsin-EDTA for $20 \mathrm{~min}$ at $37^{\circ} \mathrm{C} 5 \% \mathrm{CO}_{2}$. Cells were pelleted for $5 \mathrm{~min}$ at $650 \mathrm{~g}$ and resuspended in PBS to be immediately analyzed using a BD LSRFortessa. Were suprabasal and basal cells needed to be quantified, $2 \%$ PFA fixed cells were incubated in blocking solution (0.1\% BSA $0.5 \mathrm{mM}$ EDTA in PBS) for $15 \mathrm{~min}$ and then with anti-ITGA6-647 antibody (1:125, Biolegend, UK 313610) in blocking solution for 45 min at RT. YFP fluorescence was collected using the 488 nm laser and the 530/30 bandpass filter and ITGA6-647 fluorescence was collected using the $640 \mathrm{~nm}$ laser and the 670/14 bandpass filter. Data was analyzed using FlowJo software (version 10.5.3). Basal cells were defined as ITGA6 positive cells and suprabasal cells as ITGA6 negative cells.

\section{Immunofluorescence and microscopy}

For wholemount staining, the mouse esophagus was opened longitudinally, the muscle layer was removed and the epithelium was incubated for $1 \mathrm{~h}$ and $30 \mathrm{~min}$ in $20 \mathrm{mM}$ EDTA-PBS at $37^{\circ} \mathrm{C}$. The epithelium was peeled from underlying tissue and fixed in $4 \%$ paraformaldehyde in PBS for $30 \mathrm{~min}$. Wholemounts were blocked for $1 \mathrm{~h}$ in blocking buffer $(0.5 \%$ bovine serum albumin, $0.25 \%$ fish skin gelatin, $1 \%$ Triton $\mathrm{X}-100$ and $10 \%$ donkey serum) in PHEM buffer (60 mM PIPES, $25 \mathrm{mM}$ HEPES, $10 \mathrm{mM}$ EGTA, and $4 \mathrm{mM} \mathrm{MgSO}{ }_{4} \cdot 7 \mathrm{H}_{2} \mathrm{O}$ ). Anti-GFP antibody (1:4000, Life technologies A10262) was incubated over 3-5 days using blocking buffer, followed by several washes over $24 \mathrm{~h}$ with $0.2 \%$ Tween-20 in PHEM buffer. Where indicated, an additional overnight incubation with anti-caspase-3 (1:500, Abcam ab2302) was performed. Finally, samples were incubated for $24 \mathrm{~h}$ with $1 \mu \mathrm{g} / \mathrm{ml}$ DAPI and secondary antibody antichicken (1:2000, Jackson ImmunoResearch 703-545-155) in blocking buffer. Clones were imaged on an SP8 Leica confocal microscope. Whole tissue or large tissue area images were obtained in most cases with the $20 x$ objective with $1 x$ digital zoom, optimal pinhole and line average, speed $600 \mathrm{hz}$ and a pixel size of $0.5678 \mu \mathrm{m} /$ pixel. In YFP tissues at long time points, clones were manually detected in the microscope and individually imaged using 40x objective with $0.75 \mathrm{x}$ digital zoom, optimal pinhole and line average, speed $600 \mathrm{hz}$ and a pixel size of $0.3788 \mu \mathrm{m} / \mathrm{pixel}$. The numbers of basal and suprabasal cells in each clone were counted manually. Representative images were produced by selecting a $120 \times 120 \mu \mathrm{m}$ area in the images obtained as previously stated. Images were processed using ImageJ software adjusting brightness and contrast and applying a Gaussian blur of 1. EdU incorporation was detected with Click-iT chemistry kit according to the manufacturer's instructions (Invitrogen) using 555 Alexa Fluor azides. Confocal images for EdU-GFP staining were acquired on a 
Leica TCS SP8 confocal microscope (objective 20x; optimal pinhole and line average; speed $600 \mathrm{~Hz}$; resolution $1024 \times 1024$, zoom ×2). Images were processed using ImageJ software adjusting brightness and contrast and applying a Gaussian blur of 1 . For in vitro culture staining, inserts were fixed in $4 \%$ paraformaldehyde in PBS for $30 \mathrm{~min}$, then blocked for $30 \mathrm{~min}$ in blocking buffer and incubated overnight with anti-GFP antibody (1:1000, Life technologies A10262) in blocking buffer followed by $4 \times 15$ min washes with $0.2 \%$ Tween-20 in PHEM buffer. Finally, samples were incubated for $2 \mathrm{~h}$ with $1 \mu \mathrm{g} / \mathrm{ml} \mathrm{DAPI}$ and secondary antibody anti-chicken (1:500, Jackson ImmunoResearch 703-545-155) in blocking buffer. Afterwards, inserts were washed 4x 15 min with 0.2\% Tween-20 in PHEM buffer and mounted in Vectashield (Vector Laboratories). Cultures were imaged on an SP8 Leica confocal microscope, obtained with the $40 \mathrm{x}$ objective with $0.75 \mathrm{x}$ digital zoom, optimal pinhole and line average, speed $600 \mathrm{hz}$ and a pixel size of $0.1893 \mu \mathrm{m} /$ pixel.

\section{RNA isolation and RNA sequencing}

Total RNA was extracted from 3D cultures of mouse primary keratinocytes after 1 week in FAD medium supplemented with fetal calf serum, apo-transferrin and Penicillin/Streptomycin, with or without insulin. RNA was extracted using RNeasy Micro Kit (QIAGEN, UK), following the manufacturer's recommendations. Briefly, cells were washed with cold Hank's Balanced Salt Solution-HBSS (GIBCO, UK) and then lysis buffer was added directly to the insert. The integrity of total RNA was determined by Qubit RNA Assay Kit (Invitrogen, UK). For RNA-seq, libraries were prepared in an automated fashion using an Agilent Bravo robot with a KAPA Standard mRNA-Seq Kit (KAPA BIOSYSTEMS). In house adaptors were ligated to 100-300 bp fragments of dsDNA. All the samples were then subjected to 10 PCR cycles using sanger_168 tag set of primers and paired-end sequencing was performed on Illumina HiSeq 2500 with 75 bp read length. Reads were mapped using STAR 2.5.3a, the alignment files were sorted and duplicate-marked using Biobambam2 2.0.54, and the read summarization performed by the htseq-count script from version 0.6.1p1 of the HTSeq framework (Anders et al., 2015; Dobin et al., 2013). For GSEA analysis raw counts were normalized by the median of ratios method (Love et al., 2014). Gene set enrichment was analyzed with GSEA software (Subramanian et al., 2005) using the Hallmarks gene sets of the Molecular Signature Database (MSigDB) version 4.0 provided by the Broad Institute (http://www.broad.mit.edu/gsea/), following the standard procedure described on the GSEA user guide (http://www.broadinstitute.org/gsea/doc/GSEAUserGuideFrame.html). The FDR for GSEA is the estimated probability that a gene set with a given NES (normalized enrichment score) represents a false-positive finding, and an FDR $<0.25$ is considered to be statistically significant for GSEA. Differential gene expression was analyzed using the DEBrowser tool 
(https://debrowser.umassmed.edu/) with which we performed a DESeq2 analysis (Love et al., 2014) filtering the low counts to remove genes with less than $2 \mathrm{cpm}$ in at least 2 samples. Parametric' fitting of dispersions to the mean intensity was used with the likelihood ratio test on the difference in deviance between a full and reduced model formula (defined by nbinomLRT). An adjusted p-value cut-off of 0.05 were used to select significantly different expressed genes. Heatmaps were generated from the TPM values and build using ClustVis (https://biit.cs.ut.ee/clustvis/) and Morpheus tools (https://software.broadinstitute.org/morpheus/), significance was calculated from the adjusted p-value obtained in the DE analysis. Kyoto Encyclopedia of Genes and Genomes (KEGG) pathway enrichment analysis was performed uploading the significantly upregulated gene list $(p<0.05)$ into the Enrichr tool (https://amp.pharm.mssm.edu/Enrichr/) (Kuleshov et al., 2016). Venn diagrams were generated using the Venn Diagrams tool (https://www.biotools.fr/misc/venny). MA plots were generated using GraphPad Prism 8.

\section{Validation of Pik3ca ${ }^{H 1047 R}$ mutant construct in NIH3T3}

DNA that reflects the recombined Pik3ca ${ }^{\text {H1047R-YFP }}$ allele (PIK3CA ${ }^{\mathrm{H} 1047 \mathrm{R}}$ fused to self-cleaving peptide P2A and GFP) was chemically synthesized (GenScript USA Inc.). cDNA encoding murine $\mathrm{p} 110 \alpha^{\text {wild type }}, \mathrm{p} 110 \alpha^{\mathrm{H} 1047 \mathrm{R}}$ and $\mathrm{p} 110 \alpha^{\mathrm{H} 1047 \mathrm{R}}$-P2A-GFP were amplified by PCR using IMAGE clone (Image ID 40141870/IRCL34 C10 (M13R), Source Bioscience) for wild type $\mathrm{p} 110 \alpha$ or above cDNA for mutants and subcloned into the pCS2+ expression vector. NIH3T3 cells were transiently transfected with these constructs using Lipofectamine 2000 (Thermo Fisher 11668030) according to manufacturer's instruction. Cells were serum-starved by culture in DMEM containing $0.3 \%$ serum for $23 \mathrm{~h}$. Serum-starved NIH3T3 cells were lysed in buffer containing $20 \mathrm{mM}$ Hepes $\mathrm{NaOH}$ pH 7.9, $10 \%$ Glycerol, $0.4 \mathrm{M} \mathrm{NaCl}, 0.5 \% \mathrm{NP}-40,0.2$ $\mathrm{mM}$ EDTA, $0.01 \%$ halt protease and phosphatase inhibitor (ThermoFisher Scientific, cat \#78415). Protein concentrations were measured using standard Bradford protein assays (BioRAD QuickSTART ${ }^{\mathrm{TM}}$ Bradford Dye Reagents, cat.no.500-0202). Lysates were mixed with equal amount of $2 x$ loading buffer (100 mM Tris-HCl pH 6.8, 4\% SDS, 20\% Glycerol, Bromophenol blue and $0.2 \% \beta$-mercaptoethanol) and boiled at $96^{\circ} \mathrm{C}$ for $5 \mathrm{~min}$. Samples were loaded onto a 7.5 or $10 \%$ of SDS-polyacrylamide gel. Proteins were separated by electrophoresis and transferred onto Immobilon-P membrane (pore size $0.45 \mu \mathrm{m}$, Millipore IPVH00010). Membranes were incubated in blocking buffer ( $5 \%$ dried skimmed milk, PBS, $0.1 \%$ Tween-20) at room temperature for $1 \mathrm{~h}$ and then with primary antibodies diluted in blocking buffer for $1 \mathrm{~h}$ at room temperature or overnight at $4^{\circ} \mathrm{C}$ on a rocking platform. After washing in PBST (0.1\% Tween-20, PBS) three times, HRP conjugated secondary antibodies diluted in $0.5 \%$ skimmed milk in PBST were applied to the membrane for $10 \mathrm{~min}$ at room 
temperature on a rocking platform followed by four washes in PBST 20 min each. Washing and secondary antibodies steps were performed using SNAP id protein detection system (Sigma-Aldrich). Proteins were detected using Immobilon Western Chemiluminescent HRP substrate (Millipore WBLUC0500) or ECL blotting reagents (GE Healthcare GERPN2109).

\section{Immune capillary electrophoresis}

For protein phosphorylation analysis, 3D cultures were starved in FAD medium $0.1 \%$ FCS without cholera toxin, epidermal growth factor, insulin and hydrocortisone for $16 \mathrm{~h}$ at $37^{\circ} \mathrm{C} 5 \%$ $\mathrm{CO}_{2}$. Then treated for $15 \mathrm{~min}$ in the same starving medium or FAD medium with cholera toxin, epidermal growth factor, insulin, hydrocortisone and 20\% FCS with or without LY294002 (50 $\mu \mathrm{M}$ ). Cultures were lysed in ice-cold RIPA buffer (Thermo Scientific, UK) containing protease and phosphatase inhibitors. Plates were frozen at $-80^{\circ} \mathrm{C}$ and thawed on ice, scraped and passed twice through a Qiashredder ( 2 min centrifuged at maximum speed), then incubated $1 \mathrm{~h}$ on ice vortexing every $15 \mathrm{~min}$. Then lysates were centrifuged at $14000 \mathrm{~g}$ for $20 \mathrm{~min}$ at $4^{\circ} \mathrm{C}$. The supernatant was collected for analysis. Total protein quantification was performed using Pierce BCA Protein Assay Kit (Thermo Scientific, UK). Immune capillary electrophoresis was performed using Wes Simple ${ }^{\mathrm{TM}}$ (ProteinSimple, USA) following manufacturer's instructions. Primary antibodies used were p-AktS493 (New England Biolabs 4060S, 1:150), Akt (New England Biolabs 4691S, 1:100), p-PRAS40 (New England Biolabs 2691T, 1:300) and PRAS40 (New England Biolabs 2997T, 1:150).

\section{Respirometry experiments}

Fully recombined Pik3ca ${ }^{H 1047 R / w t}$ or uninduced (adenovirus-null infected) parallel cultures from the same mice were treated for at least 1 week in FAD medium supplemented with fetal calf serum, apo-transferrin and Penicillin/Streptomycin, with or without insulin. At the moment of the experiment, $5 \mathrm{~mm}$ circular sections of each culture were obtained using a biopsy punch (Stiefel BC-BI-1600) and transferred to XFe24 Cell Culture microplate wells with the basal layer facing up. $675 \mu$ of bicarbonate-free DMEM (Sigma-Aldrich, D5030) supplemented with $25 \mathrm{mM}$ glucose, $1 \mathrm{mM}$ pyruvate, $4 \mathrm{mM}$ glutamine, $5 \mu \mathrm{g} / \mathrm{ml}$ Apo-Transferrin with or without 5 $\mu \mathrm{g} / \mathrm{ml}$ insulin (Sigma-Aldrich 15500) was added to each sample. To eliminate residual carbonic acid from medium, cells were incubated for at least $30 \mathrm{~min}$ at $37^{\circ} \mathrm{C}$ with atmospheric $\mathrm{CO}_{2}$ in a non-humidified incubator. OCR and ECAR were assayed in a Seahorse XF-24 extracellular flux analyzer by three measurement cycles of 2-min mix, 2-min wait, and 4-min measure. Then OCR/ECAR ratio was calculated. Five technical replicates and four biological replicates were collected per condition. 


\section{Lactate measurement}

Cultures were incubated for 3 days in the specified treatments. Medium was collected and lactate was analyzed using a commercial kit (DF16, Siemens Healthcare). All sample measurements were performed by the MRC MDU Mouse Biochemistry Laboratory.

\section{Quantification and Statistical Analysis}

Unless otherwise specified, all data are expressed as mean values \pm standard deviation. Differences between groups were assessed by 2-tailed unpaired $t$-test for normally distributed data or 2-tailed Mann-Whitney $U$ test for skewed data, using GraphPad Prism software. For paired comparisons of clonal behavior across conditions, Peacock's test was used, a twodimensional extension of Kolmogorov-Smirnov test (implemented as kstest_2s_2d function for MATLAB). Exact $p$-values for statistical tests are specified in the figures. No statistical method was used to predetermine sample size. The experiments were not randomized. The investigators were not blinded to allocation during experiments and outcome assessment.

\section{Data Availability}

Raw transcriptomic data can be viewed on https://www.ebi.ac.uk/ena using the accession numbers provided in STAR methods.

\section{KEY RESOURCES TABLE}

\begin{tabular}{|l|l|l|}
\hline \multicolumn{2}{|l|}{ REAGENT or RESOURCE } & \multicolumn{2}{l|}{ SOURCE } & \multicolumn{1}{l|}{ IDENTIFIER } \\
\hline Antibodies & \multicolumn{2}{l|}{} \\
\hline Caspase 3 & Abcam & $\begin{array}{l}\text { Cat\# ab2302; RRID: } \\
\text { AB_302962 }\end{array}$ \\
\hline GFP (immunofluorescence) & Life technologies & A10262 \\
\hline GFP (western blot) & Abcam & Ab290 \\
\hline PIK3CA & Abcam & Ab152155 \\
\hline & & \\
\hline P-Akt S473 & New England Biolabs & 4060 S \\
\hline P-Akt T308 & New England Biolabs & $2965 S$ \\
\hline Akt & New England Biolabs & $4691 S$ \\
\hline PRAS40 & New England Biolabs & $2691 T$ \\
\hline P-PRAS40 & New England Biolabs & $2997 T$ \\
\hline Alexa Fluor 488 Donkey Anti-Chicken & $\begin{array}{l}\text { Jackson } \\
\text { ImmunoResearch }\end{array}$ & $\begin{array}{l}\text { Cat\# 703-545-155; } \\
\text { RRID:AB 2340375 }\end{array}$ \\
\hline $\begin{array}{l}\text { Alexa FluorRTM 647 anti-human/mouse CD49f [Clone: } \\
\text { GoH3] }\end{array}$ & Bio Legend UK Ltd & 313610 \\
\hline Goat Anti-Rabbit Immunoglobulins/HRP & Agilent-Dako & P044801-2 \\
\hline Goat Anti-Mouse Immunoglobulins/HRP & Agilent-Dako & P044701-2 \\
\hline
\end{tabular}




\begin{tabular}{|c|c|c|}
\hline \multicolumn{3}{|l|}{ Bacterial and Virus Strains } \\
\hline Adeno-CMV-iCre & Vector Laboratories & Cat\# 1045 \\
\hline Ad-CMV-Null & Vector Laboratories & Cat\# 1300 \\
\hline \multicolumn{3}{|l|}{ Chemicals, Peptides, and Recombinant Proteins } \\
\hline$\beta$-Napthoflavone & MP Biomedicals & Cat\# 156738 \\
\hline Tamoxifen & Sigma Aldrich & Cat\# N3633 \\
\hline Metformin & Sigma Aldrich & 317240 \\
\hline Sodium dichloroacetate & Sigma Aldrich & 347795 \\
\hline Fish Skin gelatin & Sigma Aldrich & Cat\# G7765 \\
\hline Bovine Serum Albumin & Merk Millipore & Cat\# 126575 \\
\hline Donkey serum & Sigma Aldrich & Cat\# D9633 \\
\hline Vectashield & Vector Lab Ltd & $\mathrm{H}-1000$ \\
\hline 40, 6-diamidino-2-phenylindole (DAPI) & Sigma Aldrich & Cat\# D9542 \\
\hline DMEM & $\begin{array}{l}\text { Thermo Fisher } \\
\text { Scientific }\end{array}$ & Cat\# 11971025 \\
\hline DMEM/F-12 & $\begin{array}{l}\text { Thermo Fisher } \\
\text { Scientific }\end{array}$ & Cat\# 31330 \\
\hline HBSS & GIBCO & Cat\#14175-053 \\
\hline Insulin & Sigma Aldrich & Cat\# I5500 \\
\hline Adenine & Sigma Aldrich & Cat\# A3159 \\
\hline Hydrocortisone & Calbiochem & Cat\# 386698 \\
\hline Cholera toxin & Sigma-Aldrich & Cat\# C8052 \\
\hline Epidermal growth factor & PeproTech & Cat\# 100-15 \\
\hline Apo-Transferrine & Sigma-Aldrich & Cat\# T2036 \\
\hline Fetal calf serum & PAA Laboratories & Cat\# A15-041 \\
\hline Penicillin-Streptomycin & Sigma Aldrich & Cat\# P0781 \\
\hline Polybrene & Sigma Aldrich & Cat\# H9268 \\
\hline Trypsin/EDTA & Sigma Aldrich & Cat\# T4174 \\
\hline PX-478 & Cambridge Bioscience & 10005189 \\
\hline RIPA buffer & $\begin{array}{l}\text { Thermo Fisher } \\
\text { Scientific }\end{array}$ & Cat\# 89900 \\
\hline Pierce BCA Protein Assay Kit & $\begin{array}{l}\text { Thermo Fisher } \\
\text { Scientific }\end{array}$ & Cat\# 23227 \\
\hline Protease and Phosphatase inhibitor & $\begin{array}{l}\text { Thermo Fisher } \\
\text { Scientific }\end{array}$ & Cat\# 78415 \\
\hline Lipofectamine 2000 & $\begin{array}{l}\text { Thermo Fisher } \\
\text { Scientific }\end{array}$ & 11668030 \\
\hline Immobilon-P membrane & Millipore & IPVH00010 \\
\hline ECL blotting reagents & GE Healthcare-Sigma & GERPN2109 \\
\hline Immobilon Western Chemiluminescent HRP substrate & Millipore & WBLUC0500 \\
\hline \multicolumn{3}{|l|}{ Critical Commercial Assays } \\
\hline Click-iT EdU imaging & Life technologies & $\begin{array}{l}\text { Cat\# C10086at\# } \\
23227\end{array}$ \\
\hline QuickSTART Bradford Dye reagents & BioRAD & Cat\# 500-0202 \\
\hline Wes Simple & ProteinSimple & Cat\# P/N 031-108 \\
\hline RNeasy Mini kit & QIAGEN & Cat\# 74106 \\
\hline Qubit RNA Assay Kit & Invitrogen & Q32852 \\
\hline \multicolumn{3}{|l|}{ Deposited Data } \\
\hline RNAseq data: Pik3cawt/wt Animal $1 \mathrm{CTL}$ & This paper & ERS2647403 \\
\hline RNAseq data: Pik3ca ${ }^{\text {wt/wt }}$ Animal $2 \mathrm{CTL}$ & This paper & ERS2647404 \\
\hline RNAseq data: Pik3ca ${ }^{\text {wt/wt }}$ Animal 3 CTL & This paper & ERS2647405 \\
\hline RNAseq data: Pik3ca ${ }^{\text {wt/wt }}$ Animal 4 CTL & This paper & ERS2647406 \\
\hline RNAseq data: Pik3ca ${ }^{H 1047 R / w t}$ Animal $1 \mathrm{CTL}$ & This paper & ERS2647407 \\
\hline
\end{tabular}


bioRxiv preprint doi: https://doi.org/10.1101/2021.05.28.446104; this version posted May 28,2021 . The copyright holder for this preprint (which was not certified by peer review) is the author/funder, who has granted bioRxiv a license to display the preprint in perpetuity. It is made available under aCC-BY 4.0 International license.

\begin{tabular}{|c|c|c|}
\hline RNAseq data: Pik3ca ${ }^{H 1047 R / w t}$ Animal 2 CTL & This paper & ERS2647408 \\
\hline RNAseq data: Pik3ca ${ }^{H 1047 R / w t}$ Animal 3 CTL & This paper & ERS2647409 \\
\hline RNAseq data: Pik3ca ${ }^{H 1047 R / w t}$ Animal 4 CTL & This paper & ERS2647410 \\
\hline RNAseq data: Pik3cawt/wt Animal 1 INS & This paper & ERS2515253 \\
\hline RNAseq data: Pik3ca ${ }^{\text {wt/wt }}$ Animal 2 INS & This paper & ERS2515254 \\
\hline RNAseq data: Pik3cawt/wt Animal 3 INS & This paper & ERS2515255 \\
\hline RNAseq data: Pik3ca ${ }^{\text {wt/wt }}$ Animal 4 INS & This paper & ERS2515256 \\
\hline RNAseq data: Pik3ca ${ }^{\mathrm{H} 1047 \mathrm{R} / \mathrm{wt}}$ Animal 1 INS & This paper & ERS2515257 \\
\hline RNAseq data: Pik3ca ${ }^{H 1047 R / w t}$ Animal 2 INS & This paper & ERS2515258 \\
\hline RNAseq data: Pik3ca ${ }^{\mathrm{H} 1047 \mathrm{R} / \mathrm{wt}}$ Animal 3 INS & This paper & ERS2515259 \\
\hline RNAseq data: Pik3ca ${ }^{H 1047 R / w t}$ Animal 4 INS & This paper & ERS2515260 \\
\hline \multicolumn{3}{|l|}{ Experimental Models: Cell Lines } \\
\hline $\mathrm{NIH} 3 \mathrm{T3}$ & ATCC & CRL-1658 \\
\hline \multicolumn{3}{|l|}{ Experimental Models: Organisms/Strains } \\
\hline Mouse: Pik3ca ${ }^{\text {fl-H1047R-T2A-EYFP-NLS/wt }}$ & This paper & $\mathrm{N} / \mathrm{A}$ \\
\hline Mouse: Ahcre & Kemp et al., 2004 & N/A \\
\hline Mouse: C57BL/6-Ins2 $2^{A k i t a / J}$ & $\begin{array}{l}\text { The Jackson } \\
\text { Laboratory }\end{array}$ & JAX: 003548 \\
\hline Mouse: Rosa26 flconfetti/ flconfetti & $\begin{array}{l}\text { The Jackson } \\
\text { Laboratory }\end{array}$ & JAX: 017492 \\
\hline Mouse: Ahcre ${ }^{E R T}$ Rosa26 fIYFP/wt & Clayton et al., 2007 & $\mathrm{~N} / \mathrm{A}$ \\
\hline \multicolumn{3}{|l|}{ Software and Algorithms } \\
\hline LAS X & Leica & N/A \\
\hline Fiji ImageJ & $\begin{array}{l}\text { Schindelin et al Nat } \\
\text { Meth } 2012\end{array}$ & https://imagej.net/Fiij \\
\hline Imaris & Bitplane & $\mathrm{N} / \mathrm{A}$ \\
\hline GraphPad & Prism 8 & N/A \\
\hline GSEA software & $\begin{array}{l}\text { Subramanian, } \\
\text { Tamayo, et al. (2005, } \\
\text { PNAS 102, 15545- } \\
15550)\end{array}$ & $\begin{array}{l}\text { https://www.gsea- } \\
\text { msigdb.org/gsea/ind } \\
\text { ex.jsp }\end{array}$ \\
\hline Morpheus & & $\begin{array}{l}\text { https://software.broa } \\
\text { dinstitute.org/morph } \\
\text { eus }\end{array}$ \\
\hline DEBrowser & & $\begin{array}{l}\text { https://debrowser.um } \\
\text { assmed.edu/ }\end{array}$ \\
\hline STAR 2.5.3a & Dobin et al., 2013 & $\mathrm{~N} / \mathrm{A}$ \\
\hline HTSeq framework version 0.6.1p1 & Anders et al., 2015 & $\mathrm{~N} / \mathrm{A}$ \\
\hline ClustVis & & $\begin{array}{l}\text { https://biit.cs.ut.ee/cl } \\
\text { ustvis/ }\end{array}$ \\
\hline Enrichr & & $\begin{array}{l}\text { https://amp.pharm.m } \\
\text { ssm.edu/Enrichr/ }\end{array}$ \\
\hline Venn Diagrams tool & & $\begin{array}{l}\text { https://www.biotools. } \\
\text { fr/misc/venny }\end{array}$ \\
\hline \multicolumn{3}{|l|}{ Other } \\
\hline Leica TCS SP8 & Leica & $\mathrm{N} / \mathrm{A}$ \\
\hline UV-irradiator CL-508M & Uvitec & $\mathrm{N} / \mathrm{A}$ \\
\hline Seahorse XFe24 Analyzer & Agilent & $\mathrm{N} / \mathrm{A}$ \\
\hline
\end{tabular}




\section{Acknowledgements}

We thank Esther Choolun and Tom Metcalf for their assistance in in vivo experiments, and Wellcome Sanger Institute RSF facilities for technical support.

This work was supported by grants from the Wellcome Trust to the Wellcome Sanger Institute (098051 and 296194) and Cancer Research UK Programme Grants to P.H.J. (C609/A17257 and C609/A27326). A.H. benefited from the award of an EMBO long term fellowship. Work in the laboratory of B.V. is supported by Cancer Research UK (C23338/A25722) and the UK NIHR University College London Hospitals Biomedical Research Centre.

\section{Author Contributions}

A.H. and B.C. performed the experiments with support from D.F-A. and C.B. K.M., B.V. and P.H.J. designed the mouse model and performed initial validations. G.P. analyzed clonal data. S.H.O. performed bioinformatics analysis. A.H., B.C. and P.H.J. wrote the manuscript with input from C.F. and B.V.

\section{Declaration of Interests}

B.V. is a consultant for Karus Therapeutics (Oxford, UK), iOnctura (Geneva, Switzerland) and Venthera (Palo Alto, CA, USA) and has received speaker fees from Gilead Sciences (Foster City, US). The authors declare no competing interests.

\section{Lead Contact and Materials Availability}

Requests for reagent and resource sharing should be addressed to the Lead Contact, Philip H. Jones (pj3@sanger.ac.uk) who will fulfil requests.

\section{Supplemental Information}

Document S1. Figures S1-S6 and Table S1. (Embedded in this manuscript)

Table S2. Source Data, Related to Figures 1, 3, 4, 5, 6, and S1-S5.

Document S2. Supplementary Theory. (Embedded in this manuscript)

Supplementary video 1.

2D simulation of the growth of wild type and Pik3ca ${ }^{H 1047 R / w t}$ clones over 6 months starting from the same proportion of induced cells and following the parameters described in Figure $3 \mathbf{I}$. 


\section{SUPPLEMENTARY THEORY}

This report provides a detailed description of the quantitative methods and modelling used to study mutant keratinocyte behavior in the murine esophagus. Section 1 covers the analysis of wild type progenitor cell behavior. In Section 2 we extend the methodology to test Pik3ca mutant progenitor cell dynamics. In Section 3 we analyze cell dynamics in the suprabasal compartment for model validation.

\section{Wild type progenitor cell dynamics}

The murine esophageal epithelium consists of layers of keratinocytes maintained by a single type of proliferative cells (Doupe et al., 2012; Piedrafita et al., 2020). Progenitor (P-) cells reside in the deepest, basal layer where they divide regularly at a rate $\lambda$. The outcome of a given progenitor division is stochastic: with a certain probability it results in two proliferating daughter cells retaining the proliferative capacity $(\mathrm{P}+\mathrm{P})$ or two post-mitotic, differentiating cells $(D+D)$, the remaining divisions yielding an asymmetric outcome $(P+D)$. Upon differentiation, D-cells stratify (at rate $\Gamma$ ) into the upper, suprabasal layers (transiting to S-cells), being ultimately shed into the lumen (at rate $\mu$ ). This scenario is summarized by the single-progenitor (SP) model (Figure 2B):

$$
\begin{aligned}
& P \stackrel{\lambda}{\rightarrow} \begin{cases}P+P & \text { Prob. } r \\
P+D & \text { Prob. } 1-2 r \\
D+D & \text { Prob. } r\end{cases} \\
& D \stackrel{\Gamma}{\rightarrow} S \\
& S \stackrel{\mu}{\rightarrow} \emptyset
\end{aligned}
$$

While the outcome of individual divisions is unpredictable, overall, the likelihood of the symmetric PP and DD division outcomes is balanced in adult wild type mice (setting the same probability, $r$ ). This ensures that on average half the progenitor cells go on to divide and half differentiate, so that the tissue remains homeostatic.

Under homeostasis, one can assume the proportion of proliferative basal cells, $\rho$, remains constant, and overall, the net rate at which cells are generated in the basal compartment is compensated by cell stratification and cell loss by shedding (Figure 2A). Then, the following relationships between the parameters can be established: $\Gamma=\lambda \rho /(1-\rho)$, and $\mu=\lambda \rho(1-$ $h) / h$, where $h$ is the proportion of suprabasal cells relative to total cells. Alternatively, one can set $\mu=\lambda \rho / m$, if we define $m$ as the global ratio of suprabasal-to-basal cell populations. 
Our lineage tracing data from Cre-RYFP (wild type) mice are consistent with key dynamical features of the SP model with balanced fates, in agreement with our more extensive work carried out previously using the same mouse strain and others (Clayton et al., 2007; Doupe et al., 2012; Piedrafita et al., 2020). First, persisting clones (i.e. those retaining at least one basal cell) show ever increasing sizes during the duration of the experiment. In particular, the average number of basal cells per persisting clone follows a linear growth over time (Figure 3B). Second, clones become increasingly heterogeneous in size, both in terms of the number of basal cells and total (basal $+1^{\text {st }}$ suprabasal) cells per clone, the distributions adopting a scaling behavior at late time points (Figure 3D). These are hallmarks of neutral clone competition, where some clones grow by chance at the expense of others that shrink, lose basal attachment and get ultimately extinct by shedding (Figure $3 \mathbf{H}$ ).

In order to validate the dynamics of the wild type progenitor cells in our particular experimental setup, we proceeded to determine the values for the unknown SP-model parameters by fitting the experimental basal clone size distributions at the different time points (suprabasal cell numbers are not required for this since proliferative cells are confined to the basal compartment). For the division rate, we took as a prior the average value for $\lambda$ and the distribution of cell-cycle time periods $t_{c c}$ inferred by H2B-GFP dilution chase experiments in (Piedrafita et al., 2020), i.e. $\left\langle\lambda>=2.9\right.$ week $^{-1}$ and $t_{c c} \sim \tau_{R}+\operatorname{Gam}(\kappa, \vartheta)$, where $\tau_{\mathrm{R}}=0.5$ day $^{-1}$ (refractory period) and Gam refers to a Gamma distribution with $\kappa=8$ and $\vartheta=\left(1-\tau_{R}<\lambda>\right.$ )$/(\kappa<\lambda>)$. Notice that the election of this realistic description of the division rate will condition the modelling implementation as this cannot rely on Poisson processes that assume independent, underlying exponential events (see below). A maximum likelihood estimation (MLE) approach was then followed to infer the values of the two other remaining parameters, $r$ and $\rho$ (which sets the value of $\Gamma$ in homeostasis), as explained below.

We performed a grid search spanning the range of all possible values for $r$ and $\rho$, and for every parameter set $\theta$ we estimated the log-likelihood value $l(\theta ; x)$ as in previous work (Figure S2G) (Murai et al., 2018; Piedrafita et al., 2020):

$$
l(\theta ; x)=\sum_{t} \sum_{n}\left(x_{n}(t) * \log p_{n}(t, \theta)\right)
$$

$x_{n}(t)$ is the observed frequency of clones with a certain basal size $n$ at time $t$. In turn, $p_{n}(t, \theta)$ refers to the probability of observing clones of that size at time $t$ given the SP model with parameter values $\theta$, and it was obtained by numerical solution ( $\geq 100,000$ simulations) of the 
Master equation. In particular, in (Murai et al., 2018), this was computed by implementing a Markov-chain Monte Carlo method (Gillespie's algorithm) (Gillespie, 1977; Gillespie, 1976). Here, we used an exact non-Markovian Monte Carlo analogue developed in (Piedrafita et al., 2020), which allows to account for the gamma-distributed cell cycle times. For convenience, both experimental and simulated clone sizes were binned in ranges increasing in powers of two given the large asymmetry of the distributions, so that in practice, $n$ in the equation above stands for clones with a number of basal cells in the range $\left(2^{n-1}+1,2^{n}\right)$.

To discard possible biases due to the initial induction of post-mitotic cells (D- or S- cells), only clones with at least two basal cells were considered for the analysis. Also, a small fraction of clones at the latest time point ( 2 out of 317 clones in the wild type; 3 out of 563 clones in the mutant) were classified as outliers (i.e. having a number of basal cells $>>2,3$ s.d. above the average of that time) and reassigned to the top size range $n$ of non-outlier clones (Murai et al., 2018; Piedrafita et al., 2020). Finally, since there were large differences in our experimental sample size across time points (especially in the wild type: 1522, 710, 457 and 317 clones quantified at time $10 \mathrm{~d}, 28 \mathrm{~d}, 84 \mathrm{~d}$ and $168 \mathrm{~d}$, respectively) and these can imprint uneven contributions to $l(\theta ; x)$ calculations (Eq. 2), here we followed a bootstrapping strategy: We computed $l(\theta ; x)$ repeatedly for different sample subsets containing a fixed number of clones $X$ across time points, drawn by random permutation with replacement of the original sampled clones. In this way we ensured even weights from the different time points and more robust parameter estimates by averaging $l(\theta ; x)$ across subsamples.

Following this methodology, we obtained the following parameter estimates $\left(\hat{\theta}_{M L E}\right)$ for the wild type progenitors (Figure 3l; Figure $\mathbf{S 2 G}$ ):

$$
\rho=0.52(0.48 ; 0.63) ; \quad r=0.08(0.07 ; 0.10)
$$

From these, we derive the stratification rate (recall homeostatic relationships above): $\Gamma=3.14$ (2.68; 4.94) week $^{-1}$. In parentheses are 95\% confidence interval bounds based on likelihoodratio test (Pearson's $\chi^{2}$ cutoff with 2 degrees of freedom). Altogether, these parameter values are in good agreement with those found previously from lineage tracing using inducible $A h$ Cre $^{E R T}$ and Lrig1-Cre ${ }^{E R T}$ based mice $(\{\rho=0.56(0.50 ; 0.89), r=0.06(0.04 ; 0.10)\}$ and $\{\rho=$ 0.65 (0.50; 0.96), $r=0.10(0.07 ; 0.15)\}$, respectively) (Piedrafita et al., 2020). It follows that using the SP model with the MLE values (Eq. 3) we obtained good fits on both the experimental average number of basal cells per clone (Figure 3B) and the distributions of 
basal clone sizes at the different time points (Figure S2I), corroborating the robust dynamics of wild type progenitors in the esophageal epithelium.

\section{Pik3ca ${ }^{H 1047 R-Y F P / w t}$ progenitor cell dynamics}

The dynamics of Pik3ca ${ }^{\text {H1047R-YFP/wt }}$ mutant progenitor cells clearly differed from wild type cells as clones showed an accelerated growth over time in both the basal and suprabasal compartments (Figure 3B-D). The percentage of $\mathrm{EdU}^{+}$basal cells among the induced Pik3ca ${ }^{\text {H1047R-YFP/wt }}$ population was similar to wild type (Figure 3F) and comparable to measurements done before in mouse esophagus (Fernandez-Antoran et al., 2019). This argues against changes in the rate of mutant cell division $\lambda$. Yet, in principle dynamics might still be explained by a SP model with balanced fates if Pik3ca ${ }^{\text {H1047R-YFP/wt }}$ progenitors experienced changes in some of the other parameters (e.g. $r$ or $\Gamma$ ). Alternatively, it could be that Pik3ca ${ }^{H 1047 R-Y F P / W t}$ clone behavior responds to an imbalance in mutant progenitor division outcomes that favors proliferating daughter cells over differentiating progeny (i.e. PP symmetric division outcome being more likely than DD) (Figure 2B). This later scenario has been shown to explain the phenotype of some other inducible mutants in squamous epithelium such as DN-Mam/1, which inhibits the Notch pathway (Alcolea et al., 2014), and Trp53 mutants (Klein et al., 2010; Murai et al., 2018). The distinction between these two possibilities is important since the former involves a neutral scenario where Pik3ca ${ }^{\text {H1047R-YFP/wt }}$ population would exhibit no competitive advantage over wild type but an exacerbated stochastic behavior (i.e. accelerated clone growth but also decline). By contrast, fate imbalance would introduce a selective advantage, cause a net exponential-like growth of mutant clones and lead to mutant cell colonization of the epithelium.

The supralinear growth observed in the average mutant clone size points towards a progenitor fate imbalance in the Pik3ca ${ }^{H 1047 R-Y F P / w t}$ population (Figure 3B). Unfortunately, however, the initial clonal induction efficiency was variable between mice and this precluded reliable confirmation of an overall mutant cell colonization. To circumvent this issue, we decided to explore the distributions of the mutant clone sizes (Figure 3D) and extend the maximum likelihood estimation (MLE) approach explained earlier to infer the most likely scenario of mutant progenitor behavior. The following model that allows for a fixed progenitor fate imbalance $(\Delta)$ was considered (Klein et al., 2010; Murai et al., 2018):

$$
P \stackrel{\lambda}{\rightarrow} \begin{cases}P+P & \text { Prob. } r(1+\Delta) \\ P+D & \text { Prob. } 1-2 r \\ D+D & \text { Prob. } r(1-\Delta)\end{cases}
$$




$$
\begin{aligned}
& D \stackrel{\Gamma}{\rightarrow} S \\
& S \stackrel{\mu}{\rightarrow} \emptyset
\end{aligned}
$$

Notice that the neutral-case scenario (balanced fates) corresponds with $\Delta=0$ (see equivalence to Eq. 1), and thus, it can be tested as a nested condition from this model. The assumption on the invariant nature of $\Delta$ is made for simplification and becomes reasonable given the low (variable) induction and the relatively short time scales in the experiments; different processes could dampen mutant fate imbalance at longer term (Colom et al., 2020; Murai et al., 2018).

The deterministic ODE equations that describe the time course of the global cell populations from the model in Eq. 4 are:

$$
\begin{aligned}
& \frac{d n_{P}}{d t}=2 \Delta r \lambda n_{P} \\
& \frac{d n_{D}}{d t}=\lambda n_{P}-2 \Delta r \lambda n_{P}-\Gamma n_{D} \\
& \frac{d n_{S}}{d t}=\Gamma n_{D}-\mu n_{S}
\end{aligned}
$$

From these, one can infer asymptotical values for $\rho$ and $h$ as $t>\Delta r \lambda$, so that the following relationships between the parameters can be established:

$$
\Gamma=\frac{\lambda \rho-2 \Delta r \lambda}{1-\rho} ; \quad \mu=\frac{\lambda \rho(1-h)-2 \Delta r \lambda}{h}
$$

A parameter grid search was done for different values of $r \in[0,0.5], \rho \in(0,1]$ and $\Delta \in[0,1]$ and stochastic model simulations run to estimate theoretical probabilities that clones develop into different sizes $p_{n}(t, \theta)$, as set earlier for the wild type (the mutant average division rate $<\lambda>$ and cell-cycle time $t_{c c}$ was assumed equal to wild type). By computing the log-likelihood value $l(\theta ; x)$ for every set of parameter values $\theta$ on the experimental distributions of the number of basal cells per clone we obtained the following parameter estimates $\left(\hat{\theta}_{M L E}\right.$ with $95 \%$ C.I.) for the Pik3ca ${ }^{H 1047 R-Y F P / w t}$ progenitors (Figure 3I; Figure S2H):

$$
\rho=0.63(0.49 ; 0.99) ; \quad r=0.17(0.12 ; 0.30) ; \quad \Delta=0.03(0.01 ; 0.06)
$$

According to the parameter relationships in Eq. 6, $\Gamma=4.86(2.72 ; 285.48)$ week $^{-1}$. It follows that independently of the values of $r$ and $\Gamma$, a neutral SP model with balanced fates $(\Delta=0)$ 
was significantly less likely to explain mutant progenitor dynamics than a model with fate imbalance $(\Delta>0)$ ), implying selection (using most favorable parameters for each model, $p=$ $0.0002,{ }^{* * *}$ by likelihood ratio test). In fact, the best neutral model found could not produce a good fit on the basal clone size distributions nor explain the curvature in the time course of the average number of basal cells per clone in the mutant population. A SP model with fate imbalance (MLE values from Eq. 7) gave an excellent fit on these experimental data (Figure 3B; Figure S2I). We conclude that Pik3ca ${ }^{\text {H1047R-YFP/wt }}$ progenitor dynamics are characterized by a small, but existent, statistical bias in fate towards an excess of dividing over differentiating daughters per average cell division. This is accompanied by an overall increased proportion of divisions being symmetric, as reflected by the larger value of $r$ (Figure 3I). Altogether, this would result in individual mutant clones developing into a wider range of possible sizes - more extreme random trajectories - while there is, overall, a relentless colonization of the esophageal epithelium by Pik3ca ${ }^{H 1047 R-Y F P / w t}$ cell population (Suppl. Video).

\section{Dynamics of suprabasal cells and total clone behavior}

A model of Pik3ca ${ }^{H 1047 R-Y F P / w t}$ selection based on progenitor fate imbalance sets some predictions on the suprabasal component of mutant clones. Therefore, within the limitations of our experimental model (Pik3ca ${ }^{\text {H1047R-YFP/wt }}$ cells could only be detected up to the $1^{\text {st }}$ suprabasal layer, above which the Pik3ca locus expression is too close to the background), we turned to study the dynamics of suprabasal and total cell populations as a validation (at least semiquantitatively) of our model findings.

First, the introduction of a fate imbalance is expected to decrease the relative proportion of suprabasal cells in the mutant clones. In particular, the global suprabasal-to-total cell ratio $h$ in the Pik3ca ${ }^{H 1047 R-Y F P / w t}$ population would decay with the value of $\Delta r$ according to the following rational function (Figure S2J) (see Eq. 6):

$$
h=\frac{\Gamma(\lambda-2 \Delta r \lambda)}{\lambda \mu+\Gamma \mu+\lambda(\Gamma-2 \Delta r \lambda)}
$$

Indeed, the average fraction of $1^{\text {st }}$ suprabasal cells in Pik3ca ${ }^{\text {H1047R-YFP/wt }}$ clones was significantly smaller than in wild type clones across time points (Figure 3G). Intriguingly, the experimental data showed a gradual decline in the proportion of suprabasal cells in both genotypes at the late time points, something a simple model with constant parameters would not capture. This apparent departure from homeostasis might be technical but could be related to epithelial changes as mice age (Liu et al., 2019). To achieve a more accurate description 
of the experimental conditions while retaining the MLE model parameters that suited basallayer behavior, we thus considered a time-dependent shedding rate $\mu(t)$, which is the only extra adjustable parameter needed to describe suprabasal cell dynamics. In this way, in the simulations of suprabasal and total clone sizes, $\mu$ could be adapted to reproduce the variable value of $h$ over time (Eq. 6).

Independently of whether we implemented time-adjusted values for $\mu$ or used a fixed default value given by the experimental average suprabasal-to-total cell ratio $h$, our (zero-parameter) model fits on the distributions of total (basal $+1^{\text {st }}$ suprabasal) cells per clone were adequate over time points (Figure 3B; Figure S2I), arguing on the adequacy of the MLE estimates.

Finally, the model predicts differences in the fraction of floating clones (i.e. clones having no basal cells) between wild type and mutant populations at intermediate time points (Figure S2K). These differences persisted regardless of whether only P-cells were considered as initially labelled or all populations were initially induced at even proportions. Floating clones reflect transient clones that have lost the proliferative capacity and are to be lost by terminal differentiation. They are expected to be less abundant if progenitors show a fate imbalance favoring proliferating over differentiating progeny. This felt in agreement with trends in the experimental data (Figure $\mathbf{3 H}$ ).

Altogether, mathematical modelling indicates Pik3ca ${ }^{H 1047 R-Y F P / w t}$ behavior is explained by mutant progenitors showing an increased ability to yield proliferating daughters upon division, which confers this genotype a competitive advantage over wild type cells.

Code used for model simulations in this study can be found at: https://github.com/gp10/DriverClonALTfate 


\section{References}

Alcolea, M.P., Greulich, P., Wabik, A., Frede, J., Simons, B.D., and Jones, P.H. (2014). Differentiation imbalance in single oesophageal progenitor cells causes clonal immortalization and field change. Nature cell biology 16, 615-622.

Ameri, K., Jahangiri, A., Rajah, A.M., Tormos, K.V., Nagarajan, R., Pekmezci, M., Nguyen, V., Wheeler, M.L., Murphy, M.P., Sanders, T.A., et al. (2015). HIGD1A Regulates Oxygen Consumption, ROS Production, and AMPK Activity during Glucose Deprivation to Modulate Cell Survival and Tumor Growth. Cell reports 10, 891-899.

Anders, S., Pyl, P.T., and Huber, W. (2015). HTSeq--a Python framework to work with highthroughput sequencing data. Bioinformatics 31, 166-169.

Banreti, A.R., and Meier, P. (2020). The NMDA receptor regulates competition of epithelial cells in the Drosophila wing. Nat Commun 11, 2228.

Barzilai, N., Crandall, J.P., Kritchevsky, S.B., and Espeland, M.A. (2016). Metformin as a Tool to Target Aging. Cell Metabolism 23, 1060-1065.

Blokzijl, F., de Ligt, J., Jager, M., Sasselli, V., Roerink, S., Sasaki, N., Huch, M., Boymans, S., Kuijk, E., Prins, P., et al. (2016). Tissue-specific mutation accumulation in human adult stem cells during life. Nature 538, 260-264.

Boucher, J., Tseng, Y.-H., and Kahn, C.R. (2010). Insulin and insulin-like growth factor-1 receptors act as ligand-specific amplitude modulators of a common pathway regulating gene transcription. J Biol Chem 285, 17235-17245.

Bradley, M.C., Ferrara, A., Achacoso, N., Ehrlich, S.F., Quesenberry, C.P., and Habel, L.A. (2018). A Cohort Study of Metformin and Colorectal Cancer Risk among Patients with Diabetes Mellitus. Cancer Epidemiology Biomarkers \&amp; Prevention 27, 525-530.

Cancer Genome Atlas Research, N., Analysis Working Group: Asan, U., Agency, B.C.C., Brigham, Women's, H., Broad, I., Brown, U., Case Western Reserve, U., Dana-Farber Cancer, I., Duke, U., et al. (2017). Integrated genomic characterization of oesophageal carcinoma. Nature 541, 169-175. Carstensen, B., Read, S.H., Friis, S., Sund, R., Keskimäki, I., Svensson, A.-M., Ljung, R., Wild, S.H., Kerssens, J.J., Harding, J.L., et al. (2016). Cancer incidence in persons with type 1 diabetes: a fivecountry study of 9,000 cancers in type 1 diabetic individuals. Diabetologia 59, 980-988.

Castel, P., Carmona, F.J., Grego-Bessa, J., Berger, M.F., Viale, A., Anderson, K.V., Bague, S., Scaltriti, M., Antonescu, C.R., Baselga, E., et al. (2016). Somatic PIK3CA mutations as a driver of sporadic venous malformations. Sci Transl Med 8, 332ra342.

Castillo, S.D., Tzouanacou, E., Zaw-Thin, M., Berenjeno, I.M., Parker, V.E., Chivite, I., Mila-Guasch, M., Pearce, W., Solomon, I., Angulo-Urarte, A., et al. (2016). Somatic activating mutations in Pik3ca cause sporadic venous malformations in mice and humans. Sci Transl Med 8, $332 \mathrm{ra3} 33$.

Chang, H.C., Kao, C.H., Chung, S.Y., Chen, W.C., Aninda, L.P., Chen, Y.H., Juan, Y.A., and Chen, S.L. (2019). Bhlhe40 differentially regulates the function and number of peroxisomes and mitochondria in myogenic cells. Redox Biology 20, 321-333.

Clayton, E., Doupe, D.P., Klein, A.M., Winton, D.J., Simons, B.D., and Jones, P.H. (2007). A single type of progenitor cell maintains normal epidermis. Nature 446, 185-189.

Colom, B., Alcolea, M.P., Piedrafita, G., Hall, M.W., Wabik, A., Dentro, S., Fowler, J.C., Herms, A., King, C., Ong, S.H., et al. (2020). Spatial competition shapes the dynamic mutational landscape of normal esophageal epithelium. Nature genetics 52, 604-614.

Consortium, T. (2017). Integrated genomic characterization of oesophageal carcinoma. Nature 541, 169-175.

de la Cova, C., Senoo-Matsuda, N., Ziosi, M., Wu, D.C., Bellosta, P., Quinzii, C.M., and Johnston, L.A. (2014). Supercompetitor status of Drosophila Myc cells requires $p 53$ as a fitness sensor to reprogram metabolism and promote viability. Cell Metab 19, 470-483.

Denko, N.C. (2008). Hypoxia, HIF1 and glucose metabolism in the solid tumour. Nature reviews Cancer 8, 705-713. 
Dobin, A., Davis, C.A., Schlesinger, F., Drenkow, J., Zaleski, C., Jha, S., Batut, P., Chaisson, M., and Gingeras, T.R. (2013). STAR: ultrafast universal RNA-seq aligner. Bioinformatics 29, 15-21.

Doupe, D.P., Alcolea, M.P., Roshan, A., Zhang, G., Klein, A.M., Simons, B.D., and Jones, P.H. (2012). A single progenitor population switches behavior to maintain and repair esophageal epithelium.

Science (New York, NY) 337, 1091-1093.

Dovmark, T.H., Saccomano, M., Hulikova, A., Alves, F., and Swietach, P. (2017). Connexin-43 channels are a pathway for discharging lactate from glycolytic pancreatic ductal adenocarcinoma cells.

Oncogene 36, 4538-4550.

Fernandez-Antoran, D., Piedrafita, G., Murai, K., Ong, S.H., Herms, A., Frezza, C., and Jones, P.H. (2019). Outcompeting p53-Mutant Cells in the Normal Esophagus by Redox Manipulation. Cell stem cell 25, 329-341.

Frede, J., Greulich, P., Nagy, T., Simons, B.D., and Jones, P.H. (2016). A single dividing cell population with imbalanced fate drives oesophageal tumour growth. Nature cell biology 18, 967-978.

Fruman, D.A., Chiu, H., Hopkins, B.D., Bagrodia, S., Cantley, L.C., and Abraham, R.T. (2017). The PI3K Pathway in Human Disease. Cell 170, 605-635.

Gillespie, D. (1977). Exact Stochastic Simulation of Coupled Chemical Reactions. The Journal of Physical Chemistry 81, 2340-2361.

Gillespie, D.T. (1976). A general method for numerically simulating the stochastic evolution of coupled chemical reactions. Journal of Computational Physics 22, 403-434.

Hall, M.W.J., Jones, P.H., and Hall, B.A. (2019). Relating evolutionary selection and mutant clonal dynamics in normal epithelia. Journal of the Royal Society Interface 16, 20190230.

Hamanaka, R.B., Glasauer, A., Hoover, P., Yang, S., Blatt, H., Mullen, A.R., Getsios, S., Gottardi, C.J., DeBerardinis, R.J., Lavker, R.M., et al. (2013). Mitochondrial reactive oxygen species promote epidermal differentiation and hair follicle development. Science signaling 6, ra8.

Hu, H., Juvekar, A., Lyssiotis, C.A., Lien, E.C., Albeck, J.G., Oh, D., Varma, G., Hung, Y.P., Ullas, S., Lauring, J., et al. (2016). Phosphoinositide 3-Kinase Regulates Glycolysis through Mobilization of Aldolase from the Actin Cytoskeleton. Cell 164, 433-446.

Ilic, N., Birsoy, K., Aguirre, A.J., Kory, N., Pacold, M.E., Singh, S., Moody, S.E., DeAngelo, J.D., Spardy, N.A., Freinkman, E., et al. (2017). <em>PIK3CA</em> mutant tumors depend on oxoglutarate dehydrogenase. Proceedings of the National Academy of Sciences 114, E3434-E3443.

Jiang, W., He, T., Liu, S., Zheng, Y., Xiang, L., Pei, X., Wang, Z., and Yang, H. (2018). The PIK3CA E542K and E545K mutations promote glycolysis and proliferation via induction of the $\beta$-catenin/SIRT3 signaling pathway in cervical cancer. Journal of Hematology \& Oncology 11, 139.

Kemp, R., Ireland, H., Clayton, E., Houghton, C., Howard, L., and Winton, D.J. (2004). Elimination of background recombination: somatic induction of Cre by combined transcriptional regulation and hormone binding affinity. Nucleic Acids Res 32, e92.

Keppler-Noreuil, K.M., Sapp, J.C., Lindhurst, M.J., Parker, V.E., Blumhorst, C., Darling, T., Tosi, L.L., Huson, S.M., Whitehouse, R.W., Jakkula, E., et al. (2014). Clinical delineation and natural history of the PIK3CA-related overgrowth spectrum. American journal of medical genetics Part A 164a, 17131733.

Kim, J.H., Bae, H.C., Kim, J., Lee, H., Ryu, W.I., Son, E.D., Lee, T.R., Jeong, S.H., and Son, S.W. (2018). HIF-1 $\alpha$-mediated BMP6 down-regulation leads to hyperproliferation and abnormal differentiation of keratinocytes in vitro. Experimental dermatology 27, 1287-1293.

Kim, J.W., Tchernyshyov, I., Semenza, G.L., and Dang, C.V. (2006). HIF-1-mediated expression of pyruvate dehydrogenase kinase: a metabolic switch required for cellular adaptation to hypoxia. Cell Metab 3, 177-185.

Klein, A.M., Brash, D.E., Jones, P.H., and Simons, B.D. (2010). Stochastic fate of <em>p53$</$ em>mutant epidermal progenitor cells is tilted toward proliferation by UV B during preneoplasia. Proceedings of the National Academy of Sciences 107, 270-275. 
Kon, S., Ishibashi, K., Katoh, H., Kitamoto, S., Shirai, T., Tanaka, S., Kajita, M., Ishikawa, S., Yamauchi, H., Yako, Y., et al. (2017). Cell competition with normal epithelial cells promotes apical extrusion of transformed cells through metabolic changes. Nature cell biology 19, 530.

Kuleshov, M.V., Jones, M.R., Rouillard, A.D., Fernandez, N.F., Duan, Q., Wang, Z., Koplev, S., Jenkins, S.L., Jagodnik, K.M., Lachmann, A., et al. (2016). Enrichr: a comprehensive gene set enrichment analysis web server 2016 update. Nucleic Acids Res 44, W90-97.

Lee-Six, H., Obro, N.F., Shepherd, M.S., Grossmann, S., Dawson, K., Belmonte, M., Osborne, R.J., Huntly, B.J.P., Martincorena, I., Anderson, E., et al. (2018). Population dynamics of normal human blood inferred from somatic mutations. Nature 561, 473-478.

Liu, N., Matsumura, H., Kato, T., Ichinose, S., Takada, A., Namiki, T., Asakawa, K., Morinaga, H., Mohri, Y., De Arcangelis, A., et al. (2019). Stem cell competition orchestrates skin homeostasis and ageing. Nature 568, 344-350.

Love, M.I., Huber, W., and Anders, S. (2014). Moderated estimation of fold change and dispersion for RNA-seq data with DESeq2. Genome biology 15, 550.

Madsen, R.R., Vanhaesebroeck, B., and Semple, R.K. (2018). Cancer-Associated PIK3CA Mutations in Overgrowth Disorders. Trends in molecular medicine 24, 856-870.

Martin-Montalvo, A., Mercken, E.M., Mitchell, S.J., Palacios, H.H., Mote, P.L., Scheibye-Knudsen, M., Gomes, A.P., Ward, T.M., Minor, R.K., Blouin, M.J., et al. (2013). Metformin improves healthspan and lifespan in mice. Nat Commun 4, 2192.

Martincorena, I., Fowler, J.C., Wabik, A., Lawson, A.R.J., Abascal, F., Hall, M.W.J., Cagan, A., Murai, K., Mahbubani, K., Stratton, M.R., et al. (2018). Somatic mutant clones colonize the human esophagus with age. Science (New York, NY) 362, 911-917.

Martincorena, I., Roshan, A., Gerstung, M., Ellis, P., Van Loo, P., McLaren, S., Wedge, D.C., Fullam, A., Alexandrov, L.B., Tubio, J.M., et al. (2015). Tumor evolution. High burden and pervasive positive selection of somatic mutations in normal human skin. Science (New York, NY) 348, 880-886.

Mboge, M.Y., Mahon, B.P., McKenna, R., and Frost, S.C. (2018). Carbonic Anhydrases: Role in pH Control and Cancer. Metabolites 8.

Michelakis, E.D., Webster, L., and Mackey, J.R. (2008). Dichloroacetate (DCA) as a potential metabolic-targeting therapy for cancer. Br J Cancer 99, 989-994.

Moore, L., Leongamornlert, D., Coorens, T.H.H., Sanders, M.A., Ellis, P., Dentro, S.C., Dawson, K.J., Butler, T., Rahbari, R., Mitchell, T.J., et al. (2020). The mutational landscape of normal human endometrial epithelium. Nature 580, 640-646.

Murai, K., Skrupskelyte, G., Piedrafita, G., Hall, M., Kostiou, V., Ong, S.H., Nagy, T., Cagan, A., Goulding, D., Klein, A.M., et al. (2018). Epidermal Tissue Adapts to Restrain Progenitors Carrying Clonal p53 Mutations. Cell stem cell 23, 687-699.e688.

Nowak, K., Seisenbacher, G., Hafen, E., and Stocker, H. (2013). Nutrient restriction enhances the proliferative potential of cells lacking the tumor suppressor PTEN in mitotic tissues. eLife 2, e00380. Oyadomari, S., Koizumi, A., Takeda, K., Gotoh, T., Akira, S., Araki, E., and Mori, M. (2002). Targeted disruption of the Chop gene delays endoplasmic reticulum stress-mediated diabetes. The Journal of clinical investigation 109, 525-532.

Piedrafita, G., Kostiou, V., Wabik, A., Colom, B., Fernandez-Antoran, D., Herms, A., Murai, K., Hall, B.A., and Jones, P.H. (2020). A single-progenitor model as the unifying paradigm of epidermal and esophageal epithelial maintenance in mice. Nat Commun 11, 1429.

Rikka, S., Quinsay, M.N., Thomas, R.L., Kubli, D.A., Zhang, X., Murphy, A.N., and Gustafsson, Å.B. (2011). Bnip3 impairs mitochondrial bioenergetics and stimulates mitochondrial turnover. Cell Death \& Differentiation 18, 721-731.

Rohwer, N., Jumpertz, S., Erdem, M., Egners, A., Warzecha, K.T., Fragoulis, A., Kuhl, A.A., Kramann, R., Neuss, S., Rudolph, I., et al. (2019). Non-canonical HIF-1 stabilization contributes to intestinal tumorigenesis. Oncogene 38, 5670-5685.

Sanaki, Y., Nagata, R., Kizawa, D., Léopold, P., and Igaki, T. (2020). Hyperinsulinemia Drives Epithelial Tumorigenesis by Abrogating Cell Competition. Developmental cell 53, 379-389.e375. 
Snippert, H.J., Schepers, A.G., van Es, J.H., Simons, B.D., and Clevers, H. (2014). Biased competition between Lgr5 intestinal stem cells driven by oncogenic mutation induces clonal expansion. EMBO Rep 15, 62-69.

Snippert, H.J., van der Flier, L.G., Sato, T., van Es, J.H., van den Born, M., Kroon-Veenboer, C., Barker, N., Klein, A.M., van Rheenen, J., Simons, B.D., et al. (2010). Intestinal crypt homeostasis results from neutral competition between symmetrically dividing Lgr5 stem cells. Cell 143, 134-144.

Srinivas, S., Watanabe, T., Lin, C.-S., William, C.M., Tanabe, Y., Jessell, T.M., and Costantini, F. (2001). Cre reporter strains produced by targeted insertion of EYFP and ECFP into the ROSA26 locus. BMC Developmental Biology 1, 4.

Subramanian, A., Tamayo, P., Mootha, V.K., Mukherjee, S., Ebert, B.L., Gillette, M.A., Paulovich, A., Pomeroy, S.L., Golub, T.R., Lander, E.S., et al. (2005). Gene set enrichment analysis: a knowledgebased approach for interpreting genome-wide expression profiles. Proc Natl Acad Sci U S A 102, 15545-15550.

Sutter, C.H., Olesen, K.M., Bhuju, J., Guo, Z., and Sutter, T.R. (2019). AHR Regulates Metabolic Reprogramming to Promote SIRT1-Dependent Keratinocyte Differentiation. J Invest Dermatol 139, 818-826.

Tello, D., Balsa, E., Acosta-Iborra, B., Fuertes-Yebra, E., Elorza, A., Ordonez, A., Corral-Escariz, M., Soro, I., Lopez-Bernardo, E., Perales-Clemente, E., et al. (2011). Induction of the mitochondrial NDUFA4L2 protein by HIF-1alpha decreases oxygen consumption by inhibiting Complex I activity. Cell Metab 14, 768-779.

Trichas, G., Begbie, J., and Srinivas, S. (2008). Use of the viral 2A peptide for bicistronic expression in transgenic mice. BMC Biol 6, 40.

Welsh, S., Williams, R., Kirkpatrick, L., Paine-Murrieta, G., and Powis, G. (2004). Antitumor activity and pharmacodynamic properties of PX-478, an inhibitor of hypoxia-inducible factor-1alpha. Mol Cancer Ther 3, 233-244.

Xie, Y., Shi, X., Sheng, K., Han, G., Li, W., Zhao, Q., Jiang, B., Feng, J., Li, J., and Gu, Y. (2019). PI3K/Akt signaling transduction pathway, erythropoiesis and glycolysis in hypoxia (Review). Mol Med Rep 19, 783-791.

Yi, M., Ban, Y., Tan, Y., Xiong, W., Li, G., and Xiang, B. (2019). 6-Phosphofructo-2-kinase/fructose-2,6biphosphatase 3 and 4: A pair of valves for fine-tuning of glucose metabolism in human cancer. Mol Metab 20, 1-13.

Ying, Z., Sandoval, M., and Beronja, S. (2018). Oncogenic activation of PI3K induces progenitor cell differentiation to suppress epidermal growth. Nature cell biology 20, 1256-1266.

Yizhak, K., Aguet, F., Kim, J., Hess, J.M., Kubler, K., Grimsby, J., Frazer, R., Zhang, H., Haradhvala, N.J., Rosebrock, D., et al. (2019). RNA sequence analysis reveals macroscopic somatic clonal expansion across normal tissues. Science (New York, NY) 364.

Yokoyama, A., Kakiuchi, N., Yoshizato, T., Nannya, Y., Suzuki, H., Takeuchi, Y., Shiozawa, Y., Sato, Y., Aoki, K., Kim, S.K., et al. (2019). Age-related remodelling of oesophageal epithelia by mutated cancer drivers. Nature 565, 312-317.

Yoshida, K., Gowers, K.H.C., Lee-Six, H., Chandrasekharan, D.P., Coorens, T., Maughan, E.F., Beal, K., Menzies, A., Millar, F.R., Anderson, E., et al. (2020). Tobacco smoking and somatic mutations in human bronchial epithelium. Nature 578, 266-272.

Yoshioka, M., Kayo, T., Ikeda, T., and Koizumi, A. (1997). A novel locus, Mody4, distal to D7Mit189 on chromosome 7 determines early-onset NIDDM in nonobese C57BL/6 (Akita) mutant mice. Diabetes 46, 887-894.

Yu, H., Zhong, X., Gao, P., Shi, J., Wu, Z., Guo, Z., Wang, Z., and Song, Y. (2019). The Potential Effect of Metformin on Cancer: An Umbrella Review. Frontiers in Endocrinology 10.

Zhang, J., Nuebel, E., Wisidagama, D.R., Setoguchi, K., Hong, J.S., Van Horn, C.M., Imam, S.S., Vergnes, L., Malone, C.S., Koehler, C.M., et al. (2012). Measuring energy metabolism in cultured cells, including human pluripotent stem cells and differentiated cells. Nat Protoc 7, 1068-1085. 
bioRxiv preprint doi: https://doi.org/10.1101/2021.05.28.446104; this version posted May 28, 2021. The copyright holder for this preprint (which was not certified by peer review) is the author/funder, who has granted bioRxiv a license to display the preprint in perpetuity. It is made available under aCC-BY 4.0 International license.

Zhang, Z., Zi, Z., Lee, E.E., Zhao, J., Contreras, D.C., South, A.P., Abel, E.D., Chong, B.F., Vandergriff, T., Hosler, G.A., et al. (2018). Differential glucose requirement in skin homeostasis and injury identifies a therapeutic target for psoriasis. Nat Med 24, 617-627. 